\title{
Cosmological milestones and Gravastars - topics in General Relativity
}

by

Céline CATTOËN

\author{
Supervisor: Dr. Matt Visser
}

A thesis submitted to the Victoria University of Wellington in fulfilment of the requirements for the degree of Master of Science in Mathematics.

Victoria University of Wellington

New Zealand 



\begin{abstract}
In this thesis, we consider two different problems relevant to general relativity. Over the last few years, opinions on physically relevant singularities occurring in FRW cosmologies have considerably changed. We present an extensive catalogue of such cosmological milestones using generalized power series both at the kinematical and dynamical level. We define the notion of "scale factor singularity" and explore its relation to polynomial and differential curvature singularities. We also extract dynamical information using the Friedmann equations and derive necessary and sufficient conditions for the existence of cosmological milestones such as big bangs, big crunches, big rips, sudden singularities and extremality events. Specifically, we provide a complete characterization of cosmological milestones for which the dominant energy condition is satisfied. The second problem looks at one of the very small number of serious alternatives to the usual concept of an astrophysical black hole, that is, the gravastar model developed by Mazur and Mottola. By considering a generalized class of similar models with continuous pressure (no infinitesimally thin shells) and negative central pressure, we demonstrate that gravastars cannot be perfect fluid spheres: anisotropc pressures are unavoidable. We provide bounds on the necessary anisotropic pressure and show that these transverse stresses that support a gravastar permit a higher compactness than is given by the Buchdahl-Bondi bound for perfect fluid stars. We also comment on the qualitative features of the equation of state that such gravastar-like objects without any horizon must have.
\end{abstract}




\section{Acknowledgment}

I would like to thank my supervisor, Dr. Matt Visser for his assistance, guidance and support. I would also like to give some special thanks to Petarpa Boonserm, Tristan Faber and Silke Weinfurtner for numerous interesting discussions relating to topics discussed in this thesis.

I wish to acknowledge the School of Mathematics, Statistics, and Computer Science for providing me with office space and all the facilities.

I am also very grateful to the Marsden fund that has indirectly supported me financially during my work at Victoria University of Wellington.

Finally, I would like to thank my family and Ian for being there for me. 


\section{Contents}

Abstract

Acknowledgment iii

1 Introduction 1

2 Some essential notions in General Relativity 5

2.1 Notions of metric, geodesics, affine connexion, Killing vector field . . . 6

2.2 Tensors . . . . . . . . . . . . . . . . . . . . . . . . . . . . . . 8

2.2.1 Riemann tensor . . . . . . . . . . . . . . . . . . 9 9

2.2.2 Ricci tensor, Ricci scalar, Weyl tensor . . . . . . . . . . . . . . . . . . . . . .

2.2 .3 Einstein tensor . . . . . . . . . . . . . . . . . . . . 10

2.2.4 Energy-momentum tensor . . . . . . . . . . . . . . 10

2.3 Einstein's field equations . . . . . . . . . . . . . . . . . 10

2.4 Weak field theory . . . . . . . . . . . . . . . . 11

2.5 Strong field theory . . . . . . . . . . . . . . . . . . . 13

2.5.1 Black holes, event horizon . . . . . . . . . . . . . . . 13

2.5.2 Schwarzschild's solution to the Einstein equations . . . . . . . 13

2.5.3 Singularities . . . . . . . . . . . . . . . . 16

2.6 Energy conditions . . . . . . . . . . . . . . . . . . . . . . . . . . . . 17

2.6.1 Null Energy condition (NEC) . . . . . . . . . . . . . . 18

2.6.2 Weak Energy condition (WEC) . . . . . . . . . . . 18

2.6.3 Strong Energy Condition (SEC) . . . . . . . . . . . . . . 19 
2.6.4 Dominant Energy Condition (DEC) . . . . . . . . . . . . 19

2.6 .5 Comments . . . . . . . . . . . . . . . . 20

2.7 Cosmology . . . . . . . . . . . . . . . . . 21

2.7.1 Introduction . . . . . . . . . . . . . . . . . 21

2.7.2 "Cosmography" . . . . . . . . . . . . . . . . 22

2.7.3 "Cosmodynamics" . . . . . . . . . . . . . . . . 22

2.7.4 Cosmological parameters . . . . . . . . . . . . . . . 23

2.8 Conclusion . . . . . . . . . . . . . . . . . . . . . . 26

$\begin{array}{lll}3 & \text { Necessary and sufficient conditions for big bangs, bounces, rips... } \quad 27\end{array}$

3.1 Introduction . . . . . . . . . . . . . . . . . . . . 27

3.2 Cosmological milestones and kinematics. . . . . . . . . . . . . . . 33

3.2 .1 Definitions . . . . . . . . . . . . . . . . . . 33

3.2 .2 Spacetime curvature . . . . . . . . . . . . . . . 42

3.3 Cosmological milestones and dynamics. . . . . . . . . . . . . . . 56

3.3 .1 Introduction . . . . . . . . . . . . . . . . . 56

$3.3 .2 \quad \mathrm{NEC} \ldots \ldots \ldots \ldots \ldots$

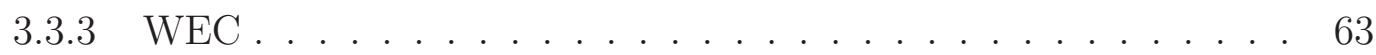

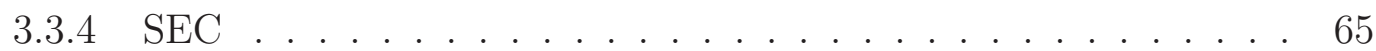

3.3 .5 DEC . . . . . . . . . . . . . . . . . 68

3.4 Total age of the universe . . . . . . . . . . . . . . . . . 74

3.4 .1 Age of the universe $\tau \ldots \ldots$. . . . . . . . . . . 77

3.4 .2 Remaining lifetime of the universe $T \ldots \ldots$. . . . . . . 80

3.4.3 Total age of the universe $T_{\text {total }} \ldots \ldots \ldots$. . . . . . . 83

3.4 .4 Summary . . . . . . . . . . . . . . . . . . 86

3.5 Results and discussion . . . . . . . . . . . . . . . . . . . 87

$\begin{array}{lll}4 & \text { Gravastars } & 91\end{array}$

4.1 Definitions . . . . . . . . . . . . . . . . . . . . . 91

4.1.1 GRAvitational VAcuum STARS . . . . . . . . . . . . 91

4.1.2 Common models . . . . . . . . . . . . . . . . . 92

4.1 .3 Problem . . . . . . . . . . . . . . . . . 93 
4.2 Properties . . . . . . . . . . . . . . . . . . 93

4.2.1 A static spherically symmetric geometry . . . . . . . . . 93

4.2 .2 A TOV equation . . . . . . . . . . . . . . . 96

4.2 .3 Specific key features . . . . . . . . . . . . . . . . . . . . . 99

4.3 The problem of the negative-pressure perfect fluid sphere gravastar. . . 104

4.3.1 Four inconsistencies . . . . . . . . . . . . . . . . . . . . 104

4.3.2 Case study: Schwarzschild interior and Buchdahl-Bondi bound . 107

4.3.3 Case study: the fate of a negative pressure perfect fluid sphere . 116

4.4 Gravastars with anisotropy . . . . . . . . . . . . . . . 120

4.4.1 Bounds on the anisotropy pressure . . . . . . . . . 121

4.4 .2 Minimizing the anisotropy . . . . . . . . . . . . . . 124

4.4.3 Features of the anisotropic equation of state . . . . . . . 127

4.5 Results and discussion . . . . . . . . . . . . . . . 130

5 Conclusion

$\begin{array}{ll}\text { A Main spacetime metrics considered } & 139\end{array}$

A.1 Static spacetime . . . . . . . . . . . . . . . . . . . . . . . 139

A.2 Schwarzschild exterior solution . . . . . . . . . . . . . . . 139

A.3 Friedmann-Robertson-Walker geometry . . . . . . . . . . . . . 140

A.4 Static spherically symmetric spacetime . . . . . . . . . . . . . 141

A.5 Interior of a gravastar . . . . . . . . . . . . . . 141

A.6 General Schwarzschild interior solution . . . . . . . . . . . . 141

A.7 Schwarzschild interior solution as a function of the mass and radius . . 142

B Necessary and sufficient conditions for big bangs, bounces, rips... 143

$\begin{array}{ll}\text { C Gravastars must have anisotropic pressures } & 145\end{array}$

$\begin{array}{ll}\text { D Effective refractive index tensor for weak-field gravity } & 147\end{array}$

$\begin{array}{ll}\text { Bibliography } & 149\end{array}$

$\begin{array}{ll}\text { Index } & 157\end{array}$ 


\section{List of Figures}

4.1 Qualitative sketch of radial pressure as a function of $r$ for a gravastar . 100

4.2 Qualitative sketch of gravastar: "core", "crust", and "atmosphere". . . 108

4.3 Qualitative sketch of pressure pole in the interior Schwarzschild solution.113

4.4 Qualitative sketch of radial pressure for a "perfect fluid" gravastar. . . 127 


\section{Chapter 1}

\section{Introduction}

This thesis is composed of three main chapters, and explores two separate problems in classical general relativity.

- The first chapter assembles some basic notions in general relativity that will be exploited in the two following research chapters.

- The second chapter is relevant to cosmology. It presents general definitions as a function of the scale factor of generic cosmological milestones (big bangs, sudden singularities...), and investigates their properties both at the kinematical and dynamical level.

- The last chapter explores the properties of a gravastar like object - that would be an alternative to black holes without any horizon.

The first problem deals with cosmology and singularities. In Friedmann-RobertsonWalker cosmologies, the physically relevant singularities had traditionally been thought to be restricted to the "big bang" or "big crunch". Even the most important "singularity theorems" (e.g. Hawking and Penrose [79, p 240]) do not specify which type of singularities are dealt with. However, in the last few years, additional different types of singularities have appeared in the literature, making the list of cosmological singularities more extensive - with big rips and sudden singularities added to the mix, as well as renewed interest in non-singular cosmological events such as bounces 
and turnarounds. We define the notion of "scale factor singularity" and define generic cosmological milestones using generalized power series of the scale factor of the universe $a(t)$. Once this classification is in place, we can investigate properties of these cosmological milestones on kinematical and dynamical levels.

- Can we find interesting relations between this notion of "scale factor singularity", and polynomial and differential curvature singularities?

- Introducing the Friedmann equations, is it possible to place constraints on whether or not the classical energy conditions are satisfied at the cosmological milestones?

Since the classification of the cosmological milestones as generalized power series of the scale factor is extremely general, the corresponding results are to a high degree model-independent. We will demonstrate that there exists a particular class of singularities that do not imply a polynomial curvature singularity near the milestone and only an even smaller sub-class of them do not imply a differential curvature singularity. We will also determine, with a minimum of technical assumptions, the necessary and sufficient conditions for the occurrences of all these cosmological milestones.

The second problem looks at an alternative model to black holes, the "gravastar model", developed by Mazur and Mottola. Instead of having a star contracting and collapsing until matter arrives at a singularity at the centre, the gravastar model suggests that a gravitationally collapsing star would force spacetime itself to undergo a phase transition that would prevent further collapse. Thus the star would be transformed into a spherical quantum vacuum surrounded by a form of super-dense matter. In the Mazur and Mottola model, the gravastar-like objects are composed of three layers (interior de Sitter space, outer region consisting of a finite-thickness shell of stiff matter, exterior Schwarzschild vacuum geometry) and two infinitesimally-thin shells with surface densities $\sigma_{ \pm}$, and surface tensions $\vartheta_{ \pm}$.

- Is it possible to replace the thin shell completely with a continuous layer of finite thickness?

- Is the pressure anisotropy (implicit in the Mazur-Mottola infinitesimally thin shell) a necessity for any gravastar-like objects? 
- Is it really possible to build a gravastar-like objects using only perfect fluid with a continuous layer of finite thickness? Could a horizon or naked singularity form and why?

Assuming pressure is continuous and differentiable, we will first analyze the resulting static geometry with the resulting isotropic TOV equation, and point out all the fatal problems arising with this situation. Secondly, we will turn to the resulting anisotropic TOV equation, and discuss when the anisotropy is necessary for the TOV equation to hold and what the resulting bounds are on transverse pressures in the anisotropic region. Finally, we will discuss what specific features the equation of state must have for an horizon-avoiding gravastar-like object to be sustained. Note that we derive these properties from an agnostic point of view as to the existence or non-existence of gravastars.

To analyze these two problems, we have made use of the theory of classical general relativity, but we have not used a quantum field theory point of view.

This thesis has three chapters but also four appendices. The first appendix exhibits all of the most important spacetime metrics used in this thesis, the last three appendices are papers published on work relating to this thesis, they were produced as a collaboration with Dr. Matt Visser, Petarpa Booserm, Tristan Faber and Silke Weinfurtner. At the time of writing, two papers ("Effective refractive index tensor for weak-field gravity") [11] and (Gravastars must have anisotropic pressures) [18] have been published in Classical Quantum Gravity, and one paper (Necessary and sufficient conditions for big bangs, bounces, crunches, rips, sudden singularities, and extremality events) [19] has been accepted for publication in Classical Quantum Gravity. 


\section{Chapter 2}

\section{Some essential notions in General Relativity}

This chapter introduces some basic notions in general relativity and cosmology. This will serve as the starting point for the specific topics investigated in this thesis. The notions of general relativity presented in this chapter are non-exhaustive.

In 1915, Einstein formulated the theory of general relativity, a theory of space, time, and gravitation. The new viewpoint it introduced on the nature of space and time often appears to be abstruse as it goes against some deeply ingrained, intuitive notions and requires some specific and sophisticated mathematic tools such as differential geometry.

Later developments, and renewed interest in general relativity began in the 1960s, when the theory was related to other areas of physics and astronomy. The modern theory of gravitational collapse, singularities, and black holes was developed at this time.

A deeper understanding of general relativity is another factor for renewed interest. Indeed, further understanding of the laws of nature are necessary to make progress toward the goal of developing a quantum theory of gravitation. Quantum gravity is the field of theoretical physics attempting to unify the theory of quantum mechanics, which three of the fundamental forces of nature, with general relativity, the theory of the fourth fundamental force: gravity. The theory of relativity in its own right 
makes many remarkable statements concerning the structure of space and time and the structure of the gravitational field.

One of the conceptual cores of general relativity is the Equivalence principle which comes in three different versions:

1. Weak Equivalence Principle: "All bodies (subject to no force other than gravity) will follow the same paths given the same initial positions and velocities." This is also often called the Principle of Uniqueness of Free Fall.

2. Einstein's (1907) Principle of Equivalence of gravitation and inertia: "All motions in an external static homogeneous gravitational field are identical to those in no gravitational field if referred to a uniformly accelerated coordinate system."

3. Strong Equivalence Principle: "At any event, always and everywhere, it is possible to choose a local inertial frame (L.I.F) such that in a sufficiently small spacetime neighbourhood all (non-gravitational) laws of nature take on their familiar forms appropriate to the absence of gravity, namely the laws of special relativity."

All the three versions are correct, but it is the Strong Equivalence Principle (now often called the Einstein Equivalence Principle) which is now viewed as the most fundamental.

Another conceptual core of general relativity is the field equations.

\subsection{Notions of metric, geodesics, affine connexion, Killing vector field}

The bending of space and time nearby an object is called a gravitational field, and its potential can be described by a spacetime metric commonly written as:

$$
d s^{2}=g_{a b} d x^{a} d x^{b} .
$$


A spacetime consists of a four-dimensional manifold $M$ and a Lorentzian metric $g_{a b}$ defined everywhere on $M$. The metric has a Lorentzian signature $(-,+,+,+)$. Indices $a$ and $b$ run from 0 (usually denoted as the time direction) to 3 . With the components of the metric $g_{a b}$, we can construct a connexion known as the affine connexion or Christoffel symbol:

$$
\Gamma_{b c}^{a}=\frac{1}{2} g^{a d}\left(g_{d b, c}+g_{d c, b}+g_{b c, d}\right)
$$

where $g^{a d}$ is the inverse metric. The connection indirectly represents the coordinate acceleration of a free falling particle in a gravitational field.

A geodesic is the curved-space generalization of the notion of a "straight line" in Euclidean space. By definition, a straight line is the path of the shortest distance between two points. More technically, a straight line is a path which parallel transports its own tangent vector.,i.e a curve whose tangent, $T^{a}$, satisfies the equation:

$$
T^{a} \nabla_{a} T^{b}=0
$$

If we consider a parametric equation of the curve $x^{a}=x^{a}(\lambda)$, we obtain the geodesic equation:

$$
\frac{d^{2} x^{a}}{d \lambda^{2}}+\Gamma_{b c}^{a} \frac{d x^{b}}{d \lambda} \frac{d x^{c}}{d \lambda}=0
$$

If we take two events $X_{1}$ and $X_{2}$, define $\triangle X=X_{2}-X_{1}$, and define the quadratic form

$$
g(\triangle X, \triangle X)=(\triangle X)^{T} g(\triangle X),
$$

we have the following definitions:

- If $g(\triangle X, \triangle X)<0$, the two events are timelike separated. It means that normal particles can successfully travel from one event to the other.

- If $g(\triangle X, \triangle X)=0$, the two events are lightlike separated. It means that one has to travel exactly at the speed of light to travel from one event to the other.

- If $g(\triangle X, \triangle X)>0$, the two events are spacelike separated. It means that one would have to travel faster than light to get from one event to the other. 
In general relativity, timelike geodesics represent spacetime motions of free falling particles and null geodesics represent the paths of light rays.

Another important mathematical concept is the one of Killing vector field. Some propositions on Killing vector fields are typically used when investigating properties related to stars (e.g. static and stationary spacetimes).

A Killing vector field is a vector field on a Riemannian manifold that preserves the metric. Killing fields are the infinitesimal generators of isometries; that is, flows generated by Killing fields are continuous isometries of the manifold. Specifically, a vector field $\xi^{a}$ is a Killing field if the Lie derivative $\mathcal{L}$ with respect to $\xi^{a}$ of the metric $g_{a b}$ vanishes:

$$
\mathcal{L}_{\xi} g_{a b}=0
$$

In terms of the covariant derivative, this is

$$
\nabla_{a} \xi_{b}+\nabla_{b} \xi_{a}=0
$$

Equation (2.7) is also known as Killing's equation, thus, the necessary and sufficient condition that $\xi^{a}$ be a Killing vector field is that it satisfy the Killing's equation.

If a spacetime has a Killing vector, then we know we can find a coordinate system in which the metric is independent of one of the coordinates. By far the most useful fact about Killing vectors is the following lemma:

Lemma 1. Let $\xi^{a}$ be a Killing vector field and let $\lambda$ be an affinely parametrized geodesic with tangent $u^{a}$. Then $\xi_{a} u^{a}$ is constant along $\lambda$.

Lemma 1 can be interpreted as saying that every Killing vector field gives rise to a conserved quantity for particles and light rays. This conserved quantity enables one to determine the gravitational redshift in stationary spacetimes and is extremely useful for integrating the geodesic equation when symmetries are present.

\subsection{Tensors}

According to Riemann's theory of curved manifolds, the geometry of space-time is completely described by the metric tensor $g_{a b}$, which has 10 algebraically independent 
components at each event.

\subsubsection{Riemann tensor}

The Riemann tensor, and its derived tensors (Ricci, Weyl), are the only tensors that can be constructed from the metric tensor and its first and second derivatives:

$$
R_{b c d}^{a}=\partial_{c} \Gamma_{b d}^{a}-\partial_{d} \Gamma_{b c}^{a}+\Gamma_{b d}^{e} \Gamma_{e c}^{a}-\Gamma_{b c}^{e} \Gamma_{e d}^{a}
$$

The Riemann curvature tensor has 20 algebraically independent components at each event. The components of the Riemann tensor identically satisfy a differential equation (the Bianchi identity (2.9)):

$$
\nabla_{a} R_{\text {debc }}+\nabla_{c} R_{\text {deab }}+\nabla_{b} R_{\text {deca }}=0
$$

The Riemann curvature tensor $R_{a b c d}$ is used to define other important tensors.

\subsubsection{Ricci tensor, Ricci scalar, Weyl tensor}

The Ricci tensor $R_{a b}$ (2.10), the Ricci scalar $R$ (2.11) and the Weyl tensor $C_{a b c d}$ for $n \geqslant 3$ dimensions (2.12), are defined by contractions, in a manner analogous to decomposing a matrix into trace and tracefree parts.

$$
\begin{gathered}
R_{a b}=g^{c d} R_{d a c b}=R_{a c b}^{c} \\
R=g^{a b} R_{a b} \\
C_{a b c d}=R_{a b c d}+\frac{1}{n-2}\left(g_{a d} R_{c b}+g_{b c} R_{d a}-g_{a c} R_{d b}-g_{b d} R_{c a}\right) \\
+\frac{1}{(n-1)(n-2)}\left(g_{a c} g_{b d}-g_{a d} g_{c b}\right) R
\end{gathered}
$$

If the Riemann tensor vanishes on a neighborhood of space-time, this neighborhood is locally isometric to Minkowski space-time, it is locally flat. Otherwise, if the Weyl tensor vanishes on a neighborhood of space-time, the neighborhood is locally conformally equivalent to Minkowski space-time. Thus, the Riemann, Ricci, and Weyl tensors all have geometric meaning independent of any physical interpretation. 


\subsubsection{Einstein tensor}

Finally, the Ricci tensor and the Ricci scalar can be used to define the Einstein tensor (2.13):

$$
G_{a b}=R_{a b}-\frac{1}{2} g_{a b} R
$$

\subsubsection{Energy-momentum tensor}

Now let's introduce the energy-momentum tensor $T^{a b}$ (also called the stress-energy tensor), this is a symmetric $T_{0}^{2}$ tensor which formulates the energy-like aspects of a system: energy density, pressure, stress, and so on.

The stress-energy tensor is defined by:

$$
T^{a b}=\left[\begin{array}{c|c}
\rho & F^{i} \\
\hline F^{j} & \Pi^{i j}
\end{array}\right],
$$

where $\rho$ is the energy density (at rest in this coordinate system), $F$ is the energy flux (generalization of the Poynting vector), and $\Pi$ is the stress tensor (pressure, shear etc...).

The three most important energy-momentum tensors in general relativity are, namely, matter or dust, perfect fluid and the electromagnetic field.

\subsection{Einstein's field equations}

We can now write the Einstein's field equations (2.15), which govern how the metric responds to energy and momentum.

$$
G_{a b}=\frac{8 \pi G}{c^{2}} T_{a b}
$$

Here $G$ is Newton's constant of universal gravitation, $G=6.67257 \times 10^{-11} \mathrm{~m}^{3} \mathrm{~kg}^{-1} \mathrm{~s}^{-2}$.

In other words, Einstein's field equation states that

$$
R_{a b}-\frac{1}{2} R g_{a b}=\frac{8 \pi G}{c^{2}} T_{a b}
$$


or equivalently

$$
R_{a b}=\frac{8 \pi G}{c^{2}}\left(T_{a b}-\frac{1}{2} g_{a b} T\right)
$$

where $T$ is the trace of the stress energy tensor. Note that often, the gravitational constant $G$ and the speed of light $c$ are set equal to one, this is called using "geometrized units".

Thus, the Ricci curvature is directly coupled to the immediate presence of matter at a given event. If there is no mass-energy at a given event, the Ricci tensor vanishes. If it were not for the Weyl tensor, this would mean that matter here could not have a gravitational influence on distant matter separated by a void. Thus, the Weyl tensor represents that part of space-time curvature which can propagate across and curve up a void.

There are two particularly physically important solutions of Einstein's field equation that represent two extremes of curvature. The Kerr vacuum solution, which models space-time outside a rotating body such as a star, has zero Ricci curvature but nonzero Weyl curvature at each event. The FRW spacetimes, which model the universe on a very large scale, has zero Weyl curvature but nonzero Ricci curvature at each event.

\subsection{Weak field theory}

When the gravitational field is "weak", general relativity can be reduced to a linearized theory. The weakness of the gravitational field allows us to decompose the metric into the flat Minkowski spacetime (2.19) of special relativity plus a small perturbation,

$$
g_{a b}=\eta_{a b}+h_{a b}, \quad\left|h_{a b}\right| \ll 1,
$$

where

$$
\eta_{a b}=\left[\begin{array}{cccc}
-1 & 0 & 0 & 0 \\
0 & 1 & 0 & 0 \\
0 & 0 & 1 & 0 \\
0 & 0 & 0 & 1
\end{array}\right]
$$


The assumption that $h_{a b}$ is small allows us to ignore anything that is higher than first order in this quantity, from which we immediately obtain:

$$
g^{a b}=\eta^{a b}+h^{a b}+O\left(h^{2}\right) ; \quad h^{a b}=\eta^{a c} \eta^{b d} h_{c d}
$$

The Christoffel symbol can now be rewritten as :

$$
\Gamma_{b c}^{a}=\frac{1}{2} \eta^{a d}\left(h_{d b, c}+h_{d c, b}-h_{b c, d}\right)+O\left(h^{2}\right) .
$$

The Riemann tensor now becomes:

$$
R_{a b c d}=-\frac{1}{2}\left(h_{a c, b d}+h_{b d, a c}-h_{a d, b c}-h_{b c, a d}\right)+O\left(h^{2}\right) .
$$

The Ricci tensor is:

$$
R_{a b}=\frac{1}{2}\left(h_{a, b c}^{c}+h_{b, a c}^{c}-\nabla^{2} h_{a b}-h_{, a b}\right)+O\left(h^{2}\right) .
$$

By making coordinate changes, we can adopt the Einstein gauge (also called de Donder gauge, or Hilbert gauge, or Fock gauge) which provides the simple result,

$$
\left[h_{b}^{c}-\frac{1}{2} h \delta_{b}^{c}\right]_{, c}=0
$$

and therefore, in this gauge, we have the simple results,

$$
R_{a b}=-\frac{1}{2} \nabla^{2} h_{a b}+O\left(h^{2}\right),
$$

and

$$
G_{a b}=-\frac{1}{2} \nabla^{2}\left(h_{a b}-\frac{1}{2} h \eta_{a b}\right)+O\left(h^{2}\right) .
$$

Thus, using the weak field limit, we can write

$$
\nabla^{2} h_{a b}=-16 \pi G\left(T_{a b}-\frac{1}{2} T \eta_{a b}\right)+O\left(h^{2}\right),
$$

which gives a lot more information. 


\subsection{Strong field theory}

Let's move from the domain of the weak field limit to solutions of the full nonlinear Einstein's equations.

\subsubsection{Black holes, event horizon}

Horizons are typically associated with strong gravitational fields (it is still possible to have a strong gravitational field without forming a horizon). By definition an event horizon is the boundary of the region from which future-directed null curves cannot escape to infinity. In other words, event horizons are "one-way membranes" permitting the passage of light and matter in only one direction and at which time slows to a stop.

Some stars (whose masses are of the order of the sun's mass), can reach a final equilibrium state, e.g. white dwarf or a neutron star. However, for stars with much larger masses, such an equilibrium state is not possible. In this case, stars will contract to such an extent that the gravitational effects will overcome the internal pressure and stresses which will not oppose the contraction. The theory of general relativity predicts that such spherically symmetric stars will necessarily contract until all matter contained in the star arrives at a singularity at the centre of the symmetry. Those specific stars are surrounded by an event horizon and are referred to as black holes.

\subsubsection{Schwarzschild's solution to the Einstein equations}

One of the most important solution is the one discovered by Schwarzschild, which describes spherically symmetric vacuum spacetimes. Since it is in vacuum, Einstein's equations become:

$$
R_{a b}=0
$$

Thus, to obtain a solution, we need to find all four-dimensional Lorentz signature metrics whose Ricci tensor vanishes and which are static and possess spherical symmetry. But first, we need to define more precisely the meaning of the terms "stationary", 
"static" and "spherically symmetric" and to choose a convenient coordinate system for analyzing this class of spacetimes.

- A stationary spacetime is a spacetime that possess a timelike Killing vector field ${ }^{1}$, $\xi^{a}$, (more precisely, a Killing vector field that is timelike near spatial infinity).

- A spacetime is static if it is stationary and, if in addition, there exists a spacelike hypersurface $\Sigma$ orthogonal to the orbits of the isometry. It is equivalent to the requirement that the timelike Killing vector field $\xi^{a}$ satisfy:

$$
\xi_{[a} \nabla_{b} \xi_{c]}=0
$$

This implies that the spacetime metric $d s^{2}$ can be chosen to be invariant under a time revearsal about the origin of time, e.g. all cross terms $d x^{t} d x^{a} \forall a \neq t$ vanish in the chosen coordinate system with arbitrary $\left\{x^{a}\right\}$ on $\Sigma$. Thus, in these coordinates, the metric components are of the form:

$$
d s^{2}=-V^{2}\left(x^{1}, x^{2}, x^{3}\right) d t^{2}+\sum_{a, b=1}^{3} g_{a b}\left(x^{1}, x^{2}, x^{3}\right) d x^{a} d x^{b},
$$

where $V^{2}=-\xi_{a} \xi^{a}$. The absence of $d t d x^{a}$ cross terms expresses the orthogonality of $\xi^{a}$ with $\Sigma$. From the explicit form of a static metric (2.30) it is clear that the diffeomorphism defined by $t \rightarrow-t$ is an isometry. And hence, in addition to the time translation symmetry, $t \rightarrow t+$ constant, possessed by all stationary spacetimes, the static spacetimes also posses a time reflection symmetry.

The static spherically symmetric metric will describe non-rotating stars or black holes, while rotating systems (which keep rotating in the same way at all times) will be described by stationary metrics.

- A spherically symmetric solution means that there exists a privileged point, e.g. the origin, such that the system is invariant under spatial rotations about the origin.

\footnotetext{
${ }^{1}$ See section 2.1 on Killing vector fields.
} 
If the spacetime is stationary and spherically symmetric, it automatically implies that it is static. An example of a stationary but non-static case is the Kerr black hole, as the rotation rate is not changing but the surfaces orthogonal to the timelike Killing field are not spacelike. For stars we will always be looking at stationary cases (and therefore static by spherical symmetry).

The Schwarzschild solution, describing static spherical symmetric vacuum spacetimes, is in standard coordinates $(t, r, \theta, \phi)$ :

$$
d s^{2}=-\left(1-\frac{2 G M}{r}\right) d t^{2}+\frac{1}{\left(1-\frac{2 G M}{r}\right)} d r^{2}+r^{2} d \Omega^{2}
$$

where $d \Omega^{2}=d \theta^{2}+\sin ^{2} \theta d \phi^{2}$. This is true for any spherically symmetric vacuum solution to Einstein's equations. $M$ is a parameter that can be interpreted as the conventional Newtonian mass that would be measured by studying orbits at large distances from the gravitating source.

As $M \longrightarrow 0$ we recover the expected Minkowski space. The metric also has the property of asymptotic flatness, as $r \longrightarrow \infty$, the metric becomes progressively Minkowskian.

Theorem 1. (Birkhoff's theorem) In a spherically symmetric spacetime the only solution to the Einstein equations (without cosmological constant) in vacuum is a piece of the Schwarzschild solution which is more properly called the "Schwarzschild exterior solution". Furthermore, a spherically symmetric vacuum solution in the exterior region is necessarily static.

There is a generalization of Theorem 1 with a cosmological constant included which leads to the Kottler solution (Schwarzschild-de Sitter spacetime). It is interesting to note that the result is a static metric but that the source was not specified except that it be spherically symmetric. Specifically, the source itself does not need to be static, it could be a collapsing star, as long as the collapse were symmetric. Practically, the metric of the Schwarzschild exterior solution corresponds to (2.31) restricted to the region $r>2 G M$. 
From the form of the Schwarzschild metric (2.31), we can see that the metric coefficients become infinite at $r=0$ and $r=2 G M$. The metric coefficients are coordinatedependent quantities, but it is certainly possible to have a coordinate singularity which results from a breakdown of a specific coordinate system rather than the underlying manifold. The metric appears to have a singularity at $r=2 G M$, but there is actually no physical singularity at that point, the coordinate system is just breaking down there. The value $r=2 m$ is known as the Schwarzschild radius ${ }^{2}$, this is a removable coordinate singularity as indicated by the Riemann tensor scalar invariant

$$
R_{a b c d} R^{a b c d}=48 m^{2} r^{-6}
$$

which is finite at $r=2 m$.

However there is an interesting physics phenomenon associated with this surface: it is the event horizon of the Schwarzschild black hole.

\subsubsection{Singularities}

The term singularity is used in many ways and in many theorems without being specifically, physically and fully explained. In this section, we will discuss some main definitions of what a singularity stand for and what it involves qualitatively.

In a manifold $M$, there can be two points which are not connected by any causal curve (timelike or lightlike) (in a Euclidean signature metric this is impossible, but not in a Lorentzian spacetime). In such a case, it is said that the geodesic runs into a singularity, which can be thought of as the edge of the manifold. Manifolds which have such singularities are known as geodesically incomplete. In fact the "singularity theorems" of Hawking and Penrose ([79, p 240]) state that, for reasonable matter content (no negative energies), spacetimes in general relativity are almost guaranteed to be geodesically incomplete.

The Big bang or the initial singularity is defined by the fact that the scale factor of the universe $a(t) \rightarrow 0$ in a finite time. This particular singularity results from a

\footnotetext{
${ }^{2}$ The value $m$ is actually interpreted as the relativistic mass with $m=G M / c^{2}$ in metres.
} 
homogeneous contraction of space down to "zero size", but does not represent an explosion of matter concentrated at a point of preexisting nonsingular spacetime. A big rip is a singularity for which the scale factor of the universe $a(t) \rightarrow \infty$ in a finite time ${ }^{3}$.

What kind of coordinate-independent feature is a warning that something goes wrong in the geometry, e.g a curvature singularity occurs? One simple criterion is when the curvature becomes infinite. The curvature is measured by the Riemann tensor from which can be constructed various scalar quantities, such as the Ricci scalar $R$, or higher order scalars $R^{a b} R_{a b}, R^{a b c d} R_{a b c d}$, etc... If any of those scalars go to infinity when approaching some point $x$, there is a curvature singularity at this point $x$.

A naked singularity is any curvature singularity not surrounded by an event horizon. Since there is no event horizon, there is no obstruction to an observer travelling to the singularity and returning to report on what was observed.

\subsection{Energy conditions}

In classical general relativity, there are several types of energy conditions [74]:

- the null energy condition (NEC);

- the weak energy condition (WEC);

- the strong energy condition (SEC);

- the dominant energy condition (DEC).

The energy conditions of general relativity permit one to deduce very powerful and general theorems about the behaviour of strong gravitational fields and cosmological geometries. In an orthonormal frame, the components of the stress energy tensor are

\footnotetext{
${ }^{3}$ For more definitions, see Chapter 3.
} 
given by:

$$
T^{\hat{a} \hat{b}}=\left[\begin{array}{cccc}
\rho & 0 & 0 & 0 \\
0 & p_{1} & 0 & 0 \\
0 & 0 & p_{2} & 0 \\
0 & 0 & 0 & p_{3}
\end{array}\right]
$$

The components of $T^{\hat{a} \hat{b}}$ are the energy density and the three principal pressures.

\subsubsection{Null Energy condition (NEC)}

For all future pointing null vectors $k^{a}$, we ask that:

$$
T_{a b} k^{a} k^{b} \geqslant 0
$$

In terms of pressures and density, we have:

$$
\forall i \quad \rho+p_{i} \geqslant 0 .
$$

Hawkings area theorem for black hole horizon relies on the NEC, and hence evaporation of a black hole must violate the NEC.

\subsubsection{Weak Energy condition (WEC)}

Sometimes it is useful to think about Einstein's equations without specifying the theory of matter from which $T^{\hat{a} \hat{b}}$ is derived. This leaves us with a great deal of arbitrariness, in the absence of some constraints on $T^{\hat{a} \hat{b}}$, any metric can satisfy the Einstein equations. The real concern is the existence of solutions to Einstein's equations with "realistic" sources of energy and momentum. The most common property that is demanded of $T^{\hat{a} \hat{b}}$ is that it represent positive energy densities - no negative masses are allowed. In a locally inertial frame this requirement can be stated as $\rho=T_{00}>0$. To turn this into a coordinate-independent statement, we ask that:

$$
T_{a b} V^{a} V^{b} \geqslant 0 \forall \text { timelike vector } V
$$

In terms of pressures and density, we have:

$$
\rho \geqslant 0 \quad \text { and } \quad \forall i \rho+p_{i} \geqslant 0 .
$$


Any timelike vector can be a tangent to an observers world line. The WEC condition states that the energy density measured by any timelike observer is non-negative. It seems like a fairly reasonable requirement, and many of the important theorems about solutions to general relativity (such as the singularity theorems of Hawking and Penrose $([79, \mathrm{p} 240]))$ rely on this condition or something very close to it. Unfortunately it is not set in stone; indeed, it is straightforward to invent otherwise respectable classical field theories which violate the WEC, and almost impossible to invent a quantum field theory which obeys it. Nevertheless, it is legitimate to assume that the WEC holds in all but the most extreme conditions.

\subsubsection{Strong Energy Condition (SEC)}

For any timelike vectors $V^{a}$, we ask that:

$$
\left(T_{a b}-\frac{T}{2} g_{a b}\right) V^{a} V^{b} \geqslant 0
$$

where $T$ is the trace of the stress-energy tensor: $T=T_{a b} g^{a b}$.

In terms of pressures and density, we have:

$$
\begin{gathered}
T=-\rho+\sum_{i} p_{i} \\
\forall i \rho+p_{i} \geqslant 0 \text { and } \rho+\sum_{i} p_{i} \geqslant 0 .
\end{gathered}
$$

Note that the SEC implies the NEC, it does not imply the WEC. For example, matter with a negative energy density but sufficiently high pressures could satisfy the SEC but would violate the WEC.

The Penrose-Hawking singularity theorem relevant to the cosmological singularity uses the SEC.

\subsubsection{Dominant Energy Condition (DEC)}

For any timelike vectors $V^{a}$, we ask that:

$$
T_{a b} V^{a} V^{b} \geqslant 0 \quad \text { and that } \quad T_{a b} V^{b} \text { is a future directed non-spacelike vector. }
$$


The DEC assumes that the WEC holds, and that for all future directed timelike vectors $V^{a}$ that $T_{a b} V^{b}$ is a future directed non-spacelike vector. This ensures that the net energy flow does not exceed the speed of light. The dominant energy condition implies the weak energy condition and also the null energy condition, but does not necessarily imply the strong energy condition.

In terms of pressures and density, we have:

$$
\rho \geqslant 0 \quad \text { and } \quad \forall i-\rho \leqslant p_{i} \leqslant \rho .
$$

The dominant energy condition can be interpreted as saying that the speed of energy flow of matter is always less than the speed of light.

\subsubsection{Comments}

Note that the null energy condition implies the weak energy condition, but otherwise the NEC, the WEC and the SEC are mathematically independent assumptions. In particular, the SEC does not imply the WEC. It is stronger only in the sense that it appears to be a stronger physical requirement to assume equation (2.6.3) rather than equation (2.6.2). Violating the NEC implies violating the DEC, SEC and WEC as well.

The energy conditions are looking a lot less secure than they once seemed:

- There are quantum effects that violate all of the energy conditions.

- There are even relatively benign looking classical systems that violate all the energy conditions [74].

Hawkings area theorem for black hole horizon relies on the NEC, and hence evaporation of a black hole must violate the NEC. 


\subsection{Cosmology}

\subsubsection{Introduction}

Cosmology is the study of the dynamical structure of the universe considered as a whole. Contemporary cosmological models are based on the idea that the universe is, on average, the same overall. This is based on a very simple principle, called "the cosmological principle", which is a generalization of the Copernican principle:

The cosmological principle: at each epoch, the universe presents the same aspect from every point, except for local irregularities [65].

When averaged over sufficiently large volumes the universe and the matter in the universe should be isotropic and homogeneous.

- Isotropy states that space looks the same no matter what direction one looks at (direction independent).

- Homogeneity is the statement that the metric is the same throughout the space (position independent).

Astronomical observations reveal that the universe is homogeneous and isotropic on the largest scales. Traditionally this homogeneity has been assumed up to "small" fluctuations that are large enough to include clusters of galaxies. The scale at which homogeneity sets in is still not completely certain. Voids with diameters of order $10^{8}$ light years are ubiquitous, forming at least $40 \%$ of the volume of the universe [48], [49], and are typically surrounded by bubble walls containing galaxy clusters. The largest feature observed - the Sloan Great Wall [40] - is $1.47 \times 10^{9}$ light years long. We simply assume homogeneity for some suitable defined cell size.

When looking at distant galaxies, they seem to be receding from our galaxy. It appears that the universe is not static, but changing with time. Thus most cosmological models are built on the fact that the universe is homogeneous and isotropic in space, but not in time. Observationally, the universe today is significantly different from the universe of $10^{10}$ years ago, and radically different from the universe of $1.5 \times 10^{10}$ years ago. 


\subsection{2 "Cosmography"}

Simply by using the assumptions of isotropy and homogeneity, a cosmological model can be derived without yet using Einstein equations. This homogeneous and isotropic cosmological model is called the Friedmann-Robertson-Walker geometry (2.35), (FLRW Friedmann-Lemaître-Robertson-Walker geometry) and is given by:

$$
d s^{2}=-d t^{2}+a(t)^{2}\left\{\frac{d r^{2}}{1-k r^{2}}+r^{2}\left[d \theta^{2}+\sin ^{2} \theta d \phi^{2}\right]\right\}
$$

where, $a(t)$ is the scale factor of the universe. There are only three values of interest for the parameter $k$ :

- $k=-1$, this corresponds to a negative curvature (for the hyperboloid)

- $k=0$, this corresponds to no curvature (flat space)

- $k=+1$, this corresponds to a positive curvature (for the 3 -sphere)

Therefore, the assumptions of homogeneity and isotropy alone have determined the spacetime metric up to three discrete possibilities of spatial geometry $k$ and the arbitrary positive function of the scale factor $a(t)$.

Observational evidence strongly suggests that our universe (or part of our universe within our causal past), is well described by a Friedmann-Robertson-Walker model, and indeed a $k=0$ model, at least as far as the decoupling time of matter and radiation.

\subsection{3 "Cosmodynamics"}

Now, by substituting the spacetime metric (2.35) into Einstein's equations (2.15), some predictions for the dynamical evolution of the system can be obtained. But first, we need to describe the matter content of the universe in terms of the stress-energy tensor. Using the assumptions of isotropy and homogeneity, the stress-energy tensor 
of matter in the present universe is approximated in an orthonormal frame by:

$$
T^{\hat{a} \hat{b}}=\left[\begin{array}{cccc}
\rho & 0 & 0 & 0 \\
0 & p & 0 & 0 \\
0 & 0 & p & 0 \\
0 & 0 & 0 & p
\end{array}\right]
$$

Here $\rho$ and $p$ are the average density and pressure due to the galaxies, stars, clouds of dusts etc...

Applying the Einstein equations imply the two following Friedmann equations:

$$
\begin{aligned}
8 \pi G_{N} \rho & =3\left[\frac{\dot{a}^{2}}{a^{2}}+\frac{k}{a^{2}}\right] \\
8 \pi G_{N} p & =-\left[\frac{\dot{a}^{2}}{a^{2}}+\frac{k}{a^{2}}+2 \frac{\ddot{a}}{a}\right] .
\end{aligned}
$$

And consequently, equation (2.37) and equation (2.38) imply:

$$
8 \pi G_{N}[\rho+3 p]=-6 \frac{\ddot{a}}{a} .
$$

The Friedmann equations completely specify the evolution of the universe as a function of time. The difficulty remains to detemermine a suitable matter model for $\rho$ and $p$, that is to make even more progress, it is necessary to choose an equation of state between $\rho$ and $p$.

\subsubsection{Cosmological parameters}

This section introduces some of the basic terminology associated with the cosmological parameters.

The rate of expansion is characterized by the Hubble parameter:

$$
H=\frac{\dot{a}}{a}
$$

The Hubble parameter quantifies the "speed" with which the size of the universe is increasing. The value of the Hubble parameter at the present epoch is the Hubble 
constant, $H_{0}$. There is currently a great deal of controversy about what its actual value is, currently the measurements ${ }^{4}$ give:

$$
H_{0}=100 h \mathrm{~km} / \mathrm{s} / \mathrm{Mpc}
$$

with a present day normalized Hubble expansion rate $h$,

$$
h=0.71_{-0.03}^{+0.04}
$$

"Mpc" stands for "megaparsec", $1 \mathrm{Mpc} \cong 3 \times 10^{24} \mathrm{~cm}$.

The universe is expanding, therefore we know that $\dot{a}>0$. From equation (2.39) we also know that $\ddot{a}<0$ when assuming that the pressure $p$ and the density $\rho$ are both positive. The universe must have been expanding at a faster and faster rate when going back in time. If we consider that the universe have always been expanding at the present rate, then at the time $T=H^{-1}=a / \dot{a}$ ago, the scale factor $a$ would be null, $a=0$. However, the expansion rate was actually faster, therefore, the time at which $a=0$ was even closer to the present. By assuming homogeneity and isotropy, general relativity makes the prediction that at a time less than $H^{-1}$ ago, the universe was in a singular state. This singular point referred to as the big bang had an infinite density of matter and an infinite curvature of spacetime.

The value of the Hubble parameter changes over time either increasing or decreasing depending on the sign of the deceleration parameter:

$$
q=-\frac{a \ddot{a}}{\dot{a}^{2}}
$$

The deceleration parameter measures the rate of change of the rate of expansion. Different values, or ranges of values, of $q_{0}$ correspond to different cosmological models. In principle, it should be possible to determine the value of $q_{0}$ observationally. For example, for a set of identical supernovae within remote galaxies, the relationship between apparent brightness and redshift is dependent on the value of the deceleration parameter. Although measurements of this kind are notoriously difficult to make and

\footnotetext{
${ }^{4}$ See S. Eidelman et al. from the Particle Data Group [29] for recent measurement values.
} 
to interpret, recent observations tend to favor accelerating universe models. In [73], it was found that

$$
q_{0}=-0.55_{-0.13}^{+0.26}
$$

Another useful quantity is the energy density parameter,

$$
\Omega=\frac{8 \pi G}{3 H^{2}} \rho=\frac{\rho}{\rho_{\text {crit }}}
$$

where the critical density (Hubble density) is

$$
\rho_{\text {crit }}=\frac{3 H^{2}}{8 \pi G} \text {. }
$$

This quantity (which will generally change with time) is called the "critical" density and current measurements ${ }^{5}$ give:

$$
\rho_{\text {crit }}=2.775 \times 10^{11} h^{2} M_{\odot} M p c^{-3}
$$

where $M_{\odot}$ is the solar mass and $h$ is the present day normalized Hubble expansion rate. Using the Friedmann equation (2.37), we can then write:

$$
\Omega-1=\frac{k}{H^{2} a^{2}} .
$$

The sign of $k$ is therefore determined by whether the energy density parameter $\Omega$ is greater than, equal to, or less than one. Indeed,

$$
\begin{aligned}
& \rho<\rho_{\text {crit }} \leftrightarrow \Omega<1 \leftrightarrow k=-1 \leftrightarrow \text { open } \\
& \rho=\rho_{\text {crit }} \leftrightarrow \Omega=1 \leftrightarrow k=0 \leftrightarrow \text { flat } \\
& \rho>\rho_{\text {crit }} \leftrightarrow \Omega>1 \leftrightarrow k=+1 \leftrightarrow \text { closed }
\end{aligned}
$$

The density parameter, then, indicates which of the three Robertson-Walker geometries describes our universe. Determining it observationally is an area of intense investigation, however, presently, it is thought to $\mathrm{be}^{6}$ :

$$
\Omega=1.02 \pm 0.02 \text {. }
$$

\footnotetext{
${ }^{5}$ See S. Eidelman et al. from the Particle Data Group [29] for recent measurement values. ${ }^{6}$ See S. Eidelman et al. from the Particle Data Group [29] for recent measurement values.
} 


\subsection{Conclusion}

This chapter recalls the main important features of general relativity that makes remarkable statements concerning the structure of space and time and the structure of the gravitational field (e.g. Einstein's equations).

The energy conditions of general relativity permit one to deduce very powerful and general theorems about the behaviour of strong gravitational fields and cosmological geometries.

A description of our universe has been outlined in section 2.7. Many important issues in cosmology remain to be solved, but it is already clear that general relativity has provided a successful picture of our universe. 


\section{Chapter 3}

\section{Necessary and sufficient conditions for big bangs, bounces, crunches, rips, sudden singularities, and extremality events}

\subsection{Introduction}

In section 2.7, we have discussed that when averaged over sufficiently large volumes the universe and the matter in the universe should be isotropic and homogeneous.

As a consequence, the cosmological principle, which is ultimately a distillation of our knowledge of observational cosmology, leads one to consider cosmological spacetimes of the idealized FRW form [17, 43, 70, 79]:

$$
\mathrm{d} s^{2}=-\mathrm{d} t^{2}+a(t)^{2}\left\{\frac{\mathrm{d} r^{2}}{1-k r^{2}}+r^{2}\left[\mathrm{~d} \theta^{2}+\sin ^{2} \theta \mathrm{d} \phi^{2}\right]\right\}
$$

where, $a(t)$ is the scale factor of the universe and should be positive, and, where, the parameter $k$ takes only three values:

- $k=-1$, negative curvature 
- $k=0$, flat space

- $k=+1$, positive curvature.

The central question in cosmology is now the prediction of the history of the scale factor $a(t)[75,76]$.

As discussed in 2.7.3, and assuming we can apply the Einstein equations of classical general relativity to cosmology in the large, we get some key dynamical equations: the Friedmann equations $(3.2,3.3,3.4,3.5)$.

Using units where $8 \pi G_{N}=1$ and $c=1$, we have:

$$
\begin{aligned}
\rho(t) & =3\left(\frac{\dot{a}^{2}}{a^{2}}+\frac{k}{a^{2}}\right), \\
p(t) & =-2 \frac{\ddot{a}}{a}-\frac{\dot{a}^{2}}{a^{2}}-\frac{k}{a^{2}}, \\
\rho(t)+3 p(t) & =-6 \frac{\ddot{a}}{a},
\end{aligned}
$$

and the related conservation equation

$$
\dot{\rho}(t) a^{3}+3[\rho(t)+p(t)] a^{2} \dot{a}=0
$$

Theorem 2. Any two of equations (3.2, 3.3, 3.4) imply the remaining one, and imply equation (3.5). Equation (3.5) and any one of equations (3.2, 3.3, 3.4) imply the two other equations but with a specific choice of integration constant.

Lemma 2. Equations (3.2) and (3.3) imply equations (3.4) and (3.5).

Proof.

$$
\begin{aligned}
\rho+3 p & =3\left(\frac{\dot{a}^{2}}{a^{2}}+\frac{k}{a^{2}}\right)-2 \frac{\ddot{a}}{a}-\frac{\dot{a}^{2}}{a^{2}}-\frac{k}{a^{2}} \\
& =\frac{1}{a^{2}}\left(3 \dot{a}^{2}+3 k-6 \ddot{a} a-3 \dot{a}^{2}-3 k\right) \\
& =-6 \frac{\ddot{a}}{a}
\end{aligned}
$$


Equation (3.8) is exactly equation (3.4). Now, using equation (3.2), we derive:

$$
\begin{aligned}
\dot{\rho}(t) & =\frac{3}{a^{4}}\left(2 a^{2} \dot{a} \ddot{a}-2 a \dot{a}^{3}-2 k a \dot{a}\right) \\
\dot{\rho}(t) a^{3} & =6 a \dot{a} \ddot{a}-6 \dot{a}^{3}-6 k \dot{a}
\end{aligned}
$$

We also have:

$$
\begin{aligned}
3(\rho+p) a^{2} \dot{a} & =3 \dot{a}(-2 a \ddot{a}+2 \dot{a}+2 k) \\
& =-6 a \dot{a} \ddot{a}+6 \dot{a}^{3}+6 k \dot{a}
\end{aligned}
$$

Now adding equations (3.10) and (3.12):

$$
\begin{aligned}
3(\rho+p) a^{2} \dot{a}+\dot{\rho}(t) a^{3} & =-6 a \dot{a} \ddot{a}+6 \dot{a}^{3}+6 k \dot{a}+6 a \dot{a} \ddot{a}-6 \dot{a}^{3}-6 k \dot{a} \\
& =0
\end{aligned}
$$

Therefore we obtain equation (3.5) from equations (3.2) and ( 3.3).

Lemma 3. Equations (3.2) and (3.4) imply equations (3.3) and (3.5).

Proof. Using equation (3.4), we can isolate $p$ and replace $\rho$ by equation (3.2):

$$
\begin{aligned}
p & =-\frac{6 \ddot{a}}{3 a}-\frac{\rho}{3}, \\
& =-2 \frac{\ddot{a}}{a}-\frac{\dot{a}^{2}}{a^{2}}-\frac{k}{a^{2}}
\end{aligned}
$$

Thus, equations (3.2) and (3.4) imply equations (3.3).

And as proven for Lemma 2., equations (3.2) and (3.3) imply equations (3.4).

Lemma 4. Equations (3.2) and (3.5) imply equations (3.3) and (3.4).

Proof. Using equation (3.2), we replace $\rho$ and $\dot{\rho}$ in equation (3.5) to obtain an equation for the pressure $p$.

$$
\begin{aligned}
\dot{\rho}(t) a^{3}+3(\rho(t)+p(t)) a^{2} \dot{a} & =0 \\
\Longrightarrow \quad 3 p a^{2} \dot{a} & =-9 \dot{a}^{3}-9 k \dot{a}-6 a \dot{a} \ddot{a}+6 \dot{a}^{3}+6 k \dot{a} \\
\Longrightarrow \quad p & =-2 \frac{\ddot{a}}{a}-\frac{\dot{a}^{2}}{a^{2}}-\frac{k}{a^{2}}
\end{aligned}
$$

Thus, equations (3.2) and (3.5) imply equations (3.3).

And as proven for Lemma 2. , equations (3.2) and (3.3) imply equations (3.4). 
Lemma 5. Equations (3.3) and (3.5) imply equations (3.2) and (3.4) up to a term proportional to an arbitrary integration constant $K: \rho \sim \frac{K}{a^{3}}$. This integration constant has to be eliminated "by hand"1.

Proof. Replacing the pressure $p$ in equation (3.5), by its value given in equation (3.3), we obtain the following differential equation:

$$
\begin{aligned}
3 \rho a^{2} \dot{a}+\dot{\rho} a^{3} & =-3 p a^{2} \dot{a} \\
3 \rho \dot{a}+\dot{\rho} a & =6 \frac{\dot{a} \ddot{a}}{a}+3 \frac{\dot{a}^{3}}{a^{2}}+3 \frac{k \dot{a}}{a^{2}} \\
\dot{\rho}+3 \frac{\dot{a}}{a} \rho & =6 \frac{\dot{a} \ddot{a}}{a^{2}}+3 \frac{\dot{a}^{3}}{a^{3}}+3 \frac{k \dot{a}}{a^{3}}
\end{aligned}
$$

By integrating this differential equation in $\rho$, we get:

$$
\rho=\frac{\int\left\{\exp \left(\int \frac{3 \dot{a}}{a} d t\right)\left(6 \frac{\dot{a} \ddot{a}}{a^{2}}+3 \frac{\dot{a}^{3}}{a^{3}}+3 \frac{k \dot{a}}{a^{3}}\right)\right\} d t+K}{\exp \left(\int \frac{3 \dot{a}}{a} d t\right)}
$$

where $K$ is an integration constant. Furthermore,

$$
\exp \left(\int \frac{3 \dot{a}}{a} d t\right)=\exp (3 \ln (a))=a^{3},
$$

and therefore,

$$
\begin{aligned}
\int\left\{\exp \left(\int \frac{3 \dot{a}}{a} d t\right)\left(\frac{3 \rho \dot{a}+\dot{\rho} a}{a}\right)\right\} d t & =\int\left\{a^{3}\left(\frac{3 \rho \dot{a}+\dot{\rho} a}{a}\right)\right\} d t \\
& =\int\left(6 a \dot{a} \ddot{a}+3 \dot{a}^{3}+3 k \dot{a}\right) d t \\
& =3 a \dot{a}^{2}+3 k a
\end{aligned}
$$

We finally have:

$$
\rho=3\left(\frac{\dot{a}^{2}}{a^{2}}+\frac{k}{a^{2}}+\frac{K}{3 a^{3}}\right)
$$

The last term $\frac{K}{3 a^{3}}$ of equation (3.28) has to be eliminated "by hand" to obtain exactly equation (3.2).

And as proven for Lemma 2., equations (3.2) and (3.3) imply equations (3.4).

\footnotetext{
${ }^{1}$ Physically, this extra term $\frac{K}{a^{3}}$ corresponds to "dust".
} 
Lemma 6. Equations (3.4) and (3.5) imply equations (3.2) and (3.3) with $k$ arising as an integration constant and being set to $k=-1 / 0 /+1$ by suitably rescaling $a(t)$.

Proof. Using equations (3.4) and (3.5), we get:

$$
\begin{aligned}
\dot{\rho}(t) a^{3}+3\left(\rho(t)+\left(-2 \frac{\ddot{a}}{a}-\frac{\dot{a}^{2}}{a^{2}}-\frac{k}{a^{2}}\right)\right) a^{2} \dot{a} & =0 \\
\Longrightarrow \quad \dot{\rho}+2 \frac{\dot{a}}{a} \rho & =6 \frac{\dot{a} \ddot{a}}{a^{2}}
\end{aligned}
$$

By integrating the differential equation, we obtain:

$$
\rho=\frac{\int\left\{\exp \left(\int 2 \frac{\dot{a}}{a} d t\right) 6 \frac{\dot{a} a}{a^{2}}\right\} d t+K}{\exp \left(\int 2 \frac{\dot{a}}{a} d t\right)},
$$

where $K$ is an integration constant. Furthermore,

$$
\exp \left(\int 2 \frac{\dot{a}}{a} d t\right)=\exp (2 \ln (a))=a^{2},
$$

and therefore,

$$
\begin{aligned}
\rho & =\frac{\int 6 \dot{a} \ddot{a} d t+K}{a^{2}} \\
& =\frac{\int 3 \frac{d \dot{a}^{2}}{d t}+K}{a^{2}} \\
& =\frac{3 \dot{a}^{2}+K}{a^{2}}
\end{aligned}
$$

By suitably rescaling $a(t)$, we can, without loss of generality set this integration constant to $k=-1 / 0 /+1$. That is

- if $K=0$, then we do not need to rescale $a(t)$.

- if $K \neq 0$, then we can define $a(t)_{\text {new }}=a(t) / \sqrt{|K|}$. After this substitution, we get

$$
\begin{aligned}
\rho & =\frac{3\left(\dot{a}_{\text {new }} \sqrt{|K|}\right)^{2}+K}{\left(a_{\text {new }} \sqrt{|K|}\right)^{2}} \\
& =3\left(\frac{\dot{a}_{\text {new }}^{2}}{a_{\text {new }}^{2}}+\frac{K}{|K| a_{\text {new }}^{2}}\right) \\
& =3\left(\frac{\dot{a}_{\text {new }}^{2}}{a_{\text {new }}^{2}}+\frac{k}{a_{\text {new }}^{2}}\right),
\end{aligned}
$$


with the only possible values of $k=-1 / 0 /+1$.

Finally, we get the expected equation:

$$
\rho=3\left(\frac{\dot{a}^{2}}{a^{2}}+\frac{k}{a^{2}}\right)
$$

And as proven for Lemma 3., equations (3.2) and (3.4) imply equations (3.3).

The physically relevant singularities occurring in the Friedmann-Robertson-Walker cosmologies had traditionally been thought to be limited to the "big bang", and possibly a "big crunch". However, over the last few years, the zoo of cosmological singularities considered in the literature has become considerably more extensive, with "big rips" and "sudden singularities" added to the mix, as well as renewed interest in non-singular cosmological events such as "bounces" and "turnarounds"2.

Typically, those singularities are classified in relation to what happens to the scale factor $a(t)$ of the universe at the time when the singularity occurs.

- For a big bang and a big crunch, $a(t) \longrightarrow 0$.

- For a big rip, $a(t) \longrightarrow \infty$.

- For a sudden singularity, the definition varies according to different authors: $a(t)$ is finite at the time of the occurrence of the singularity (everyone agrees on that), however, the first derivative of the scale factor is infinite $\dot{a}(t) \longrightarrow \infty$, or it could be the second derivative $\ddot{a}(t) \longrightarrow \infty$, etc..., depending on how "sudden" one is willing to describe a sudden singularity.

- For bounces and turnarounds, $a(t)$ is finite and reaches either a minimum or a maximum.

- For points of inflexion, $a(t)$ is finite and reaches a stationary point.

\footnotetext{
${ }^{2}$ See references $[6,7,8,12,15,16,22,23,32,34,35,52,63,69]$. Examples of sudden singularities have been given earlier, for example in references [55], [56], in which they are referred to as "Crack of Doom" singularities.
} 


\section{Problem:}

Is it possible to come up with generic definitions that would apply to a complete catalogue of such cosmological milestones, both at the kinematical and dynamical level?

- Kinematics:

Can we define a notion of "scale-factor singularity" and find interesting relations between this notion and curvature singularities?

- Dynamics:

Using the Friedmann equations (without assuming even the existence of any equation of state) is it possible to place constraints on whether or not the classical energy conditions are satisfied at the cosmological milestones?

In this chapter, we use these considerations to derive necessary and sufficient conditions for the existence of cosmological milestones such as bangs, bounces, crunches, rips, sudden singularities, and extremality events. Since the classification is extremely general, the corresponding results are to a high degree model-independent: in particular, we determine, with a minimum of technical assumptions, the necessary and sufficient conditions for the occurrences of all theses cosmological milestones. Note that we only use classical general relativity to derive those results.

\subsection{Cosmological milestones and kinematics.}

\subsubsection{Definitions}

In this section, we develop precise definitions of a hopefully complete catalogue of cosmological milestones.

It is clear that it would be really convenient to have some unspecified generic cosmological milestone defined in terms of the behaviour of the scale factor $a(t)$, and which occurs at some finite time $t_{\odot}$. 
Generalized Frobenius series are commonly used when expanding solutions of differential equations around their singular points. Motivated by this property, we will assume that in the vicinity of the milestone the scale factor has a generalized power series expansion that generalizes the notions of Taylor series, meromorphic Laurent series, Frobenius series, and Liapunov expansions [54] and are even more general than the generalized Frobenius series adopted in [78]. Indeed, in the present context, if the scale factor $a(t)$ is representable by such a generalized power series, then by the Friedmann equations both $\rho(t)$ and $p(t)$ are representable by such power series. Formal reversion of the power series then implies that the equation of state $\rho(p)$, and thence also the function $\rho(a)$ possess such generalized power series. Conversely, if $\rho(a)$ is representable by such a generalized power series, then by the first Friedmann equation, $\dot{a}(t)$ has a power series of this type, which upon integration implies that $a(t)$ itself possesses such a power series. Similarly, if the equation of state $p(\rho)$ is representable by such a generalized power series then by integrating the conservation equation we have

$$
a(\rho)=a_{*} \exp \left\{\frac{1}{3} \int_{\rho_{*}}^{\rho} \frac{\mathrm{d} \bar{\rho}}{\bar{\rho}+p(\bar{\rho})}\right\},
$$

which will now also possess such a generalized power series. That an extension of the usual concept of a Frobenius series is likely to be useful is already clear from the analysis of [78]. We should also be clear concerning what type of object falls outside this class of generalized power series: First, essential singularities [effectively poles of infinite order, arising for example in functions such as $\exp (-1 / x)$ considered in the neighbourhood of $x=0$ ] lie outside this classification, and secondly, certain variations on the notion of Puiseux series [specifically, series containing $(\ln x)^{n},(\ln \ln x)^{n},(\ln \ln \ln x)^{n}, \ldots$ ] also lie outside this classification. We are not aware of any situations in which these exceptional cases become physically relevant.

Definition 1. Generic finite-time cosmological milestones: Suppose we have some unspecified generic cosmological milestone, that is defined in terms of the behaviour of the scale factor $a(t)$, and which occurs at some finite time $t_{\odot}$. We will assume that in the vicinity of the milestone the scale factor has a (possibly one-sided) generalized 
power series expansion of the form

$$
a(t)=c_{0}\left|t-t_{\odot}\right|^{\eta_{0}}+c_{1}\left|t-t_{\odot}\right|^{\eta_{1}}+c_{2}\left|t-t_{\odot}\right|^{\eta_{2}}+c_{3}\left|t-t_{\odot}\right|^{\eta_{3}}+\ldots
$$

where the indicial exponents $\eta_{i}$ are generically real (but are often non-integer) and without loss of generality are ordered in such a way that they satisfy

$$
\eta_{0}<\eta_{1}<\eta_{2}<\eta_{3} \ldots
$$

Finally we can also without loss of generality set

$$
c_{0}>0
$$

There are no a priori constraints on the signs of the other $c_{i}$, though by definition $c_{i} \neq 0$.

From a physical point of view, this definition is really generic and can be applied to any type of cosmological milestone. This generalized power series expansion is sufficient to encompass all the models we are aware of in the literature, and as a matter of fact, the indicial exponents $\eta_{i}$ will be used to classify the type of cosmological milestone we are dealing with. For many of the calculations in this chapter, the first term in the expansion is dominant, but even for the most subtle of the explicit calculations below it will be sufficient to keep only the first three terms of the expansion:

$$
a(t)=c_{0}\left|t-t_{\odot}\right|^{\eta_{0}}+c_{1}\left|t-t_{\odot}\right|^{\eta_{1}}+c_{2}\left|t-t_{\odot}\right|^{\eta_{2}} \ldots ; \quad \eta_{0}<\eta_{1}<\eta_{2} ; \quad c_{0}>0 .
$$

The lowest few of the indicial exponents are sufficient to determine the relationship between these cosmological milestones, the curvature singularities and even the energy conditions of classical general relativity.

Note that this expansion fails if the cosmological milestone is pushed into the infinite past or infinite future. We shall return to this point later when we discuss the total age of the universe. 


\section{Big Bangs and big crunches}

Big bangs and big crunches are some of the most basic cosmological milestones encountered in the literature. For these types of singularities, the scale factor $a(t) \rightarrow 0$ at some finite time as one moves to the past or future.

According to the Big Bang theory, the universe originated in an infinitely dense and physically paradoxical singularity. Space has expanded with the passage of time, objects being moved farther away from each other. In cosmology, the Big Bang theory is the prevailing theory about the early development and shape of the universe. The central idea is that the observation that galaxies appear to be receding from each other can be combined with the theory of general relativity to extrapolate the conditions of the universe back in time. This leads to the conclusion that as one goes back in time, the universe becomes increasingly hot and dense. However, the big bang represents the creation of the universe from a singular state, not explosion of matter into a pre-existing spacetime. It might be hoped that the perfect symmetry of the FRW universes was responsible for this singularity, but however, the singularity theorems predict (under relatively mild conditions) that any universe with $\rho>0$ and $p \geqslant 0$ must have begun at a singularity.

There are a number of consequences to this view. One consequence is that the universe now is very different than the universe in the past or in the future. The Big Bang theory predicts that at some point, the matter in the universe was hot and dense enough to prevent light from flowing freely in space. That this period of the universe would be observable in the form of cosmic background radiation (CBR) was first predicted in the 1940s, and the discovery of such radiation in the 1960s swung most scientific opinion against the Big Bang theory's chief rival, the steady state theory.

In cosmology, the Big Crunch is a hypothesis that states the universe will stop expanding and start to collapse upon itself; a counterpart to the Big Bang. If the gravitational attraction of all the matter in the observable horizon is high enough, then it could stop the expansion of the universe, and then reverse it. The universe would then contract, in about the same time as the expansion took. Eventually, all matter and energy would be compressed back into a gravitational singularity. It is meaningless to ask what would happen after this, as time would stop in this singular- 
ity as well.

We shall define the scale factor near a big bang and a big crunch using the generalized power series expansion mentioned earlier.

Definition 2. Let the time of the big bang (if one occurs) be denoted by $t_{*}$ and the time of the big crunch (if one occurs) be denoted by $t_{\circledast}$. We shall say that the bang or crunch behaves with indicial exponents $\left(0<\eta_{0}<\eta_{1} \ldots\right)$ if the scale factor possesses a generalized power series in the vicinity of the singularity. That is, if

$$
a(t)=c_{0}\left(t-t_{*}\right)^{\eta_{0}}+c_{1}\left(t-t_{*}\right)^{\eta_{1}}+\ldots
$$

or

$$
a(t)=c_{0}\left(t_{\circledast}-t\right)^{\eta_{0}}+c_{1}\left(t_{\circledast}-t\right)^{\eta_{1}}+\ldots
$$

respectively. Moreover, the series have been carefully constructed to make $a\left(t_{*}\right)=0$ and $a\left(t_{\circledast}\right)=0$.

\section{Big rips}

A "big rip" is a singularity for which $a(t) \rightarrow \infty$ at finite time. A big rip could occur in the future or in the past, however, the literature to date has solely considered future rips (as a past rip would be a most unusual and unexpected beginning to the history of the universe).

The Big Rip is a cosmological hypothesis about the ultimate fate of the Universe. The key to this hypothesis is the amount of dark energy in the universe. If the universe contains enough dark energy, it could end with all matter being pulled apart. First the galaxies would be separated from each other, then gravity would be too weak to hold individual galaxies together.

We shall write the scale factor near a big rip using the generalized power series expansion.

Definition 3. Let the time of the rip, if it occurs, be denoted $t_{*}$, then we define the indicial exponents of the rip (either future or past) to be $\left(\eta_{0}<\eta_{1} \ldots\right)$ if the scale factor 
possesses a generalized power series in the vicinity of the rip:

$$
a(t)=c_{0}\left|t_{*}-t\right|^{\eta_{0}}+c_{1}\left|t_{*}-t\right|^{\eta_{1}}+\ldots,
$$

with $\eta_{0}<0$ and $c_{0}>0$. Moreover, the series has been carefully constructed to make $a\left(t_{*}\right)=\infty$.

Note the similarity to bangs and crunches, with the only difference being in the sign of the exponent $\eta_{0}$.

\section{Sudden singularities}

"Sudden singularities" are a recent type of cosmological milestones that have appeared in the literature. There could be past or future sudden singularities for which some time derivative of the scale factor diverges at finite time, while the scale factor itself remains finite. Again, future sudden singularities are more popular than past sudden singularities as those ones would be a most unusual and disturbing beginning to the history of the universe.

Recently it has been speculated that in an expanding FLRW universe a curvature singularity may occur at a finite time before a "Big Crunch" for matter contents that satisfy both weak and strong energy conditions. This family of models has been further enlarged, and the same sort of behavior has also been found in inhomogeneous models $[6,7]$.

We shall define the scale factor near a sudden singularity using the generalized power series expansion.

Definition 4. Let the time of the sudden singularity, if one occurs, be $t_{\ominus}$ (past or future). A suitable definition of the exponent of a sudden singularity is to take $\eta_{0}=0$ and $\eta_{1}>0$ to give

$$
a(t)=c_{0}+c_{1}\left|t-t_{\Theta}\right|^{\eta_{1}}+\ldots
$$

with $c_{0}>0$ and $\eta_{1}$ non-integer. Thus $a\left(t_{\odot}\right)=c_{0}$ is finite and a sufficient number of differentiations yields

$$
a^{(n)}\left(t \rightarrow t_{\ominus}\right) \sim c_{0} \eta_{1}\left(\eta_{1}-1\right)\left(\eta_{1}-2\right) \ldots\left(\eta_{1}-n+1\right)\left|t-t_{\Theta}\right|^{\eta_{1}-n} \rightarrow \infty .
$$


The toy model considered by Barrow $[7,6,8]$ can be written

$$
a(t)=c_{0}\left[\left(t_{\odot}-t\right)^{\eta}-1\right]+\tilde{c}_{0}\left(t-t_{*}\right)^{\tilde{\eta}}
$$

and falls into this classification when expanded around the time of the sudden singularity, $t_{\odot}$, while it falls into the classification of big bang singularities considered above when expanded around the time of the big bang, $t_{*}$.

\section{Extremality events}

Let's consider some other common cosmological milestones (which are not singularities in any sense) that are referred to as "extremality events". In particular, for these events, the scale factor $a(t)$ exhibits a local extremum at finite time, and whence, $\dot{a} \rightarrow 0$. In the vicinity of extremality events we can model the scale factor using ordinary Taylor series so that in terms of our generalized series we have $\eta_{0}=0$ and $\eta_{i} \in Z^{+}$.

Definition 5. A "bounce" is any local minimum of a(t), the time of such an event being denoted by $t_{\bullet}$, so that $a^{(1)}\left(t_{\bullet}\right)=0$. The "order" of the bounce is the first nonzero integer $n$ for which the $2 n$ 'th time derivative is strictly positive:

$$
a^{(2 n)}\left(t_{\bullet}\right)>0
$$

so that

$$
a(t)=a\left(t_{\bullet}\right)+\frac{1}{(2 n) !} a^{(2 n)}\left(t_{\bullet}\right)\left[t-t_{\bullet}\right]^{2 n}+\ldots
$$

Note that $a^{(1)}\left(t_{\bullet}\right)=0$ at the bounce is in agreement with $[2,9,13,36,39,47,50$, $60,62,64,71,72]$ and that the $2 n$ 'th time derivative strictly positive is also mentioned in $[62,47]$.

Definition 6. A "turnaround" is any local maximum of a(t), the time of such an event being denoted by $t_{0}$, so that $a^{(1)}\left(t_{\circ}\right)=0$. The "order" of the turnaround is the first nonzero integer $n$ for which the $2 n$ 'th time derivative is strictly negative:

$$
a^{(2 n)}\left(t_{\circ}\right)<0
$$


so that

$$
a(t)=a\left(t_{\circ}\right)+\frac{1}{(2 n) !} a^{(2 n)}\left(t_{\circ}\right)\left[t-t_{\circ}\right]^{2 n}+\ldots
$$

Note that turnarounds are mentioned in $[5,14,33,61]$.

Definition 7. An "inflexion event" is an extremality event that is neither a local maximum or a local minimum, the time of such an event being denoted by $t_{\odot}$. (Inflexion events can be thought of as an extreme case of "loitering", in the limit where the Hubble parameter momentarily vanishes at the inflexion event.) The order of the inflexion event is the first nonzero $n$ for which

$$
a^{(2 n+1)}\left(t_{\odot}\right) \neq 0
$$

so that

$$
a(t)=a\left(t_{\odot}\right)+\frac{1}{(2 n+1) !} a^{(2 n+1)}\left(t_{\odot}\right)\left[t-t_{\odot}\right]^{2 n+1}+\ldots
$$

The loitering universe scenario is an expanding Friedmann cosmology that undergoes a fairly recent phase of slow expansion. It is during this semi-static phase that large-scale structure would be formed. Loitering is also characterized by the fact that the Hubble parameter dips in value over a narrow redshift range referred to as the "loitering epoch". During loitering, density perturbations would be expected to grow rapidly, and, since the expansion of the universe would slow down, its age near loitering would dramatically increase [66, 67] .

Definition 8. The "emergent universe" of [31, 30] can be thought of as an extremality event that has been pushed back into the infinite past.

The "emergent universe" scenario consists of an inflationary universe that would emerge from a small static state that has within it the seeds for the development of the macroscopic universe. The universe would have a finite initial size, with a finite amount of inflation occurring over an infinite time in the past, and with inflation would then coming to an end via reheating in the standard way. The scale-factor would be bounded away from zero in the past and there would be no horizon problem and no singularity, since the initial state would be Einstein static. Also, the initial static state 
could be chosen to have a radius above the Planck scale, so that these models could even avoid a quantum gravity regime [31, 30].

These definitions have been chosen to match with and simplify the definitions in articles [47] and [62]. Note that for bounces these definitions imply that there will be some open interval such that

$$
\forall t \in\left(t_{\bullet}-\Delta, t_{\bullet}\right) \cup\left(t_{\bullet}, t_{\bullet}+\Delta\right) ; \quad \ddot{a}(t)>0,
$$

while for turnarounds there will be some open interval such that

$$
\forall t \in\left(t_{\circ}-\Delta, t_{\circ}\right) \cup\left(t_{\circ}, t_{\circ}+\Delta\right) ; \quad \ddot{a}(t)<0 .
$$

Note that unless the bounce or turnaround is of order one we cannot guarantee that at the extremality event itself $\ddot{a}\left(t_{\bullet}\right)>0$ or $\ddot{a}\left(t_{\circ}\right)<0$. For inflexion events we can only assert the weaker condition of the existence of some open interval such that

$$
\forall t \in\left(t_{\odot}-\Delta, t_{\odot}\right) \cup\left(t_{\odot}, t_{\odot}+\Delta\right) ; \quad \dot{a}(t) \text { has fixed sign. }
$$

\section{Summary}

We have defined singular cosmological milestones (big bang, big crunch, big rip, sudden singularity) and nonsingular cosmological milestones (extremality events) using a single framework based on generalized power series. From a kinematical point of view, major features will be deduced with the first two indicial exponents $\left(\eta_{0}\right.$ and $\left.\eta_{1}\right)$.

The value of the first indicial exponent $\eta_{0}$ relates to the classification of the types of singular cosmological milestones as follows:

- $\eta_{0}>0$ for big bangs or big crunches.

- $\eta_{0}=0$ for sudden singularities.

- $\eta_{0}<0$ for big rips.

For the nonsingular cosmological milestones (extremality events), the parameterization is simpler: only one positive integer is needed, i.e. the order of the extremality event, to determine the qualitative behaviour of the Taylor series of the scale factor $a(t)$. 


\subsubsection{Spacetime curvature}

In this section, we will use the parameters define above to explore generic properties of the cosmological milestones. Do all cosmological milestones with scale factor singularities lead to curvature singularities?

\section{Cosmological parameters}

Before analyzing the spacetime curvature, we consider two cosmological parameters:

- the Hubble parameter $H=\frac{\dot{a}}{a}$, characterizing the rate of expansion ${ }^{3}$.

- the deceleration parameter ${ }^{4} q=-\frac{\ddot{a}}{a} H^{-2}=-\frac{a \ddot{a}}{\dot{a}^{2}}$.

Theorem 3. Consider a generalized power series expansion of the scale factor as defined in Definition 1, for $\eta_{0} \neq 0$, that is for bangs, crunches, and rips, the Hubble parameter exhibits a generic $1 /\left(t-t_{\odot}\right)$ blow up, where $t_{\odot}$ is the the time of the event. Explicitly,

$$
\lim _{t \rightarrow t_{\odot}^{+}} H= \begin{cases}+\infty & \eta_{0}>0 \\ -\infty & \eta_{0}<0 .\end{cases}
$$

Proof. From the definition of a generic cosmological milestone

$$
a(t)=c_{0}\left|t-t_{\odot}\right|^{\eta_{0}}+c_{1}\left|t-t_{\odot}\right|^{\eta_{1}}+\ldots
$$

we have (assuming $t>t_{\odot}$ for simplicity, otherwise one need merely formally reverse the flow of time $)^{5}$

$$
\dot{a}(t)=c_{0} \eta_{0}\left(t-t_{\odot}\right)^{\eta_{0}-1}+c_{1} \eta_{1}\left(t-t_{\odot}\right)^{\eta_{1}-1}+\ldots
$$

\footnotetext{
${ }^{3}$ See section 2.7.4.

${ }^{4}$ See section 2.7.4.

${ }^{5}$ In fact for explicit calculations in the vicinity of any cosmological milestone it is always possible to choose the direction of time to force $t>t_{\odot}$ and so dispense with the need to take the absolute value $\left|t-t_{\odot}\right|$, at least for one-sided calculations. This is not a physical restriction on the cosmological milestone, just a mathematical convenience which we shall adopt henceforth without further explicit discussion.
} 
Keeping only the most dominant term, we have for $\eta_{0} \neq 0$

$$
H=\frac{\dot{a}}{a} \sim \frac{c_{0} \eta_{0}\left(t-t_{\odot}\right)^{\eta_{0}-1}}{c_{0}\left(t-t_{\odot}\right)^{\eta_{0}}}=\frac{\eta_{0}}{t-t_{\odot}} ; \quad\left(\eta_{0} \neq 0\right) .
$$

That is, for bangs, crunches, and rips the Hubble parameter exhibits a generic $1 /\left(t-t_{\odot}\right)$ blow up. For $\eta_{0}>0, \lim _{t \rightarrow t_{\odot}} \eta_{0} /\left(t-t_{\odot}\right)=+\infty$ whereas, for $\eta_{0}<0$, $\lim _{t \rightarrow t_{\odot}} \eta_{0} /\left(t-t_{\odot}\right)=-\infty$.

Theorem 3 implies that for the particular cosmological milestones such as big bangs, big crunches, and big rips, the Hubble parameter goes to infinity in the vicinity of the time of the event.

Theorem 4. Consider a generalized power series expansion of the scale factor as defined in Definition 1, for $\eta_{0}=0$, that is either a sudden singularity or an extremality event, the Hubble parameter does not necessarily diverge at the cosmological milestone. As a matter of fact,

$$
\lim _{t \rightarrow t_{\odot}} H=\left\{\begin{array}{lll}
0 & \eta_{0}=0 ; & \eta_{1}>1 ; \\
c_{1} / c_{0} & \eta_{0}=0 ; & \eta_{1}=1 ; \\
\operatorname{sign}\left(c_{1}\right) \infty & \eta_{0}=0 ; & \eta_{1} \in(0,1) ;
\end{array}\right.
$$

where $t_{\odot}$ is the the time of the event.

Proof. For $\eta_{0}=0$, we need to go to the next highest term in the numerator (a term which depends on $\eta_{1}$, which is guaranteed to be greater than zero by our definitions) to obtain

$$
H \sim \frac{c_{1} \eta_{1}\left(t-t_{\odot}\right)^{\eta_{1}-1}}{c_{0}}=\eta_{1} \frac{c_{1}}{c_{0}}\left(t-t_{\odot}\right)^{\eta_{1}-1} ; \quad\left(\eta_{0}=0 ; \eta_{1}>0\right) .
$$

In particular, this guarantees that power law behaviour is completely generic near the cosmological milestone, and while the value of the exponent is typically -1 , there is an exceptional class of milestones (the sudden singularities and extremality events) for which the exponent will differ. It is not automatic that the Hubble parameter diverge at the cosmological milestone. In fact,

$$
\lim _{t \rightarrow t_{\odot}} H=\eta_{1} \frac{c_{1}}{c_{0}}\left(t-t_{\odot}\right)^{\eta_{1}-1}=\left\{\begin{array}{lll}
0 & \eta_{0}=0 ; & \eta_{1}>1 \\
c_{1} / c_{0} & \eta_{0}=0 ; & \eta_{1}=1 ;
\end{array}\right.
$$


Theorem 4 implies that for the particular cosmological milestones such as sudden singularities and extremality events, the Hubble parameter does not necessary diverge and in particular, the Hubble parameter has a finite limit iff $\eta_{0}=0, \eta_{1} \geq 1$, corresponding to a particular subset of the sudden singularities.

The following theorem is a summary of the results encountered in Theorem 3 and Theorem 4.

\section{Theorem 5. Summary:}

Consider a generalized power series expansion of the scale factor as defined in Definition 1, the Hubble parameter does not necessarily diverge at every different cosmological milestone. Indeed,

$$
\lim _{t \rightarrow t_{\odot}} H=\left\{\begin{array}{lll}
+\infty & \eta_{0}>0 ; & \\
0 & \eta_{0}=0 ; & \eta_{1}>1 ; \\
c_{1} / c_{0} & \eta_{0}=0 ; & \eta_{1}=1 ; \\
\operatorname{sign}\left(c_{1}\right) \infty & \eta_{0}=0 ; & \eta_{1} \in(0,1) ; \\
-\infty & \eta_{0}<0, &
\end{array}\right.
$$

where $t_{\odot}$ is the the time of the event.

Proof. Theorem 3 and Theorem 4 implies Theorem 5.

Now that we have analyzed the Hubble parameter in detail, we will consider the so-called deceleration parameter $q=-\frac{\ddot{a}}{a} H^{-2}=-\frac{a \ddot{a}}{\dot{a}^{2}}$ and give some similar results.

Theorem 6. Consider a generalized power series expansion of the scale factor as defined in Definition 1, the deceleration parameter $q$ can be either finite or infinite as the cosmological milestone is approached:

- For bangs, crunches, and rips, $\left(\eta_{0} \neq 0\right)$ the limit of $q$ is always finite.

- For a certain subset of the sudden singularities $\left(\eta_{0}=0\right)$ the limit $q$ is infinite. 
Indeed,

$$
\lim _{t \rightarrow t_{\odot}} q=\left\{\begin{array}{llll}
\left(1-\eta_{0}\right) / \eta_{0} & \eta_{0} \neq 0 ; & \\
\operatorname{sign}\left(c_{1}\left[1-\eta_{1}\right]\right) \infty & \eta_{0}=0 ; & \eta_{1} \neq 1 ; & \\
0 & \eta_{0}=0 ; & \eta_{1}=1 ; & \eta_{2}>2 ; \\
-2 c_{2} c_{0} / c_{1}^{2} & \eta_{0}=0 ; & \eta_{1}=1 ; & \eta_{2}=2 ; \\
-\operatorname{sign}\left(c_{2}\right) \infty & \eta_{0}=0 ; & \eta_{1}=1 ; & \eta_{2} \in(1,2),
\end{array}\right.
$$

where $t_{\odot}$ is the the time of the event.

Proof. First, we will consider the cosmological acceleration $\ddot{a}$ :

$\ddot{a}(t)=c_{0} \eta_{0}\left(\eta_{0}-1\right)\left|t-t_{\odot}\right|^{\eta_{0}-2}+c_{1} \eta_{1}\left(\eta_{1}-1\right)\left|t-t_{\odot}\right|^{\eta_{1}-2}+c_{2} \eta_{2}\left(\eta_{2}-1\right)\left|t-t_{\odot}\right|^{\eta_{2}-2}+\ldots$

Remember that for the scale factor $a(t)$, we have:

$$
a(t)=c_{0}\left|t-t_{\odot}\right|^{\eta_{0}}+c_{1}\left|t-t_{\odot}\right|^{\eta_{1}}+c_{2}\left|t-t_{\odot}\right|^{\eta_{2}}+c_{3}\left|t-t_{\odot}\right|^{\eta_{3}}+\ldots
$$

Then provided $\eta_{0} \neq 0$ and $\eta_{0} \neq 1$

$$
\frac{\ddot{a}}{a} \sim \frac{\eta_{0}\left(\eta_{0}-1\right)}{\left(t-t_{\odot}\right)^{2}}
$$

If $\eta_{0}=0$, then provided $\eta_{1} \neq 1$

$$
\frac{\ddot{a}}{a} \sim \frac{\eta_{1}\left(\eta_{1}-1\right) c_{1}}{c_{0}}\left(t-t_{\odot}\right)^{\eta_{1}-2}
$$

If both $\eta_{0}=0$ and $\eta_{1}=1$

$$
\frac{\ddot{a}}{a} \sim \frac{\eta_{2}\left(\eta_{2}-1\right) c_{2}}{c_{0}}\left(t-t_{\odot}\right)^{\eta_{2}-2}
$$

Finally, if $\eta_{0}=1$, then (since $\eta_{1}>1$ )

$$
\frac{\ddot{a}}{a} \sim \frac{\eta_{1}\left(\eta_{1}-1\right) c_{1}}{c_{0}}\left(t-t_{\odot}\right)^{\eta_{1}-3}
$$

The behaviour of $\ddot{a} / a$ near the milestone will be some power law, though the precise exponent of that power law will depend on the interplay between the various indicial 
exponents $\eta_{i}$. Note that there is at least one situation in which we have to calculate up to the third exponent $\eta_{2}$. We can now consider the so-called deceleration parameter

$$
q=-\frac{\ddot{a}}{a} H^{-2}=-\frac{a \ddot{a}}{\dot{a}^{2}}
$$

Following the same sort of analysis, for $\eta_{0} \neq 0$ and $\eta_{0} \neq 1$ we have the "generic" result:

$$
q \sim \frac{1-\eta_{0}}{\eta_{0}} ; \quad\left(\eta_{0} \neq 0,1\right)
$$

For the "exceptional" cases we easily see:

- If $\eta_{0}=0$, then provided $\eta_{1} \neq 1$

$$
q \sim-\frac{\left(\eta_{1}-1\right) c_{0}}{\eta_{1} c_{1}}\left(t-t_{\odot}\right)^{-\eta_{1}}
$$

- If both $\eta_{0}=0$ and $\eta_{1}=1$

$$
q \sim-\frac{c_{2} c_{0}}{c_{1}^{2}} \eta_{2}\left(\eta_{2}-1\right)\left(t-t_{\odot}\right)^{\eta_{2}-2}
$$

- Finally, if $\eta_{0}=1$, then (since $\eta_{1}>1$ )

$$
q \sim-\frac{\eta_{1}\left(\eta_{1}-1\right) c_{1}}{c_{0}}\left(t-t_{\odot}\right)^{\eta_{1}-1}
$$

Again we see the ubiquity of power law behaviour, with a "generic" case and a limited number of special cases. Using the generic case and the special cases, we can calculate the limit of the deceleration parameter at the cosmological milestone. For $\eta_{0} \neq 0$ and $\eta_{0} \neq 1$ we get the "generic" result:

$$
\lim _{t \rightarrow t_{\odot}} q=\left(1-\eta_{0}\right) / \eta_{0} \quad\left(\eta_{0} \neq 0,1\right)
$$

For the "exceptional" cases we get:

- If $\eta_{0}=0$, then provided $\eta_{1} \neq 1$

$$
\lim _{t \rightarrow t_{\odot}} q=\operatorname{sign}\left(c_{1}\left[1-\eta_{1}\right]\right) \infty .
$$


- If both $\eta_{0}=0$ and $\eta_{1}=1$

$$
\lim _{t \rightarrow t_{\odot}} q= \begin{cases}0 & \eta_{2}>2 ; \\ -2 c_{2} c_{0} / c_{1}^{2} & \eta_{2}=2 ; \\ -\operatorname{sign}\left(c_{2}\right) \infty & \eta_{2} \in(1,2) .\end{cases}
$$

- Finally, if $\eta_{0}=1$, then (since $\eta_{1}>1$ )

$$
\lim _{t \rightarrow t_{\odot}} q=0 .
$$

Note that in this case, the next dominant term $\eta_{1}$ goes to 0 as $t \rightarrow t_{\odot}$. We can therefore write this limit as

$$
\lim _{t \rightarrow t_{\odot}} q=\left(1-\eta_{0}\right) /\left.\eta_{0}\right|_{\eta_{0}=1}=0
$$

Hence, we have for the deceleration parameter:

$$
\lim _{t \rightarrow t_{\odot}} q=\left\{\begin{array}{llll}
\left(1-\eta_{0}\right) / \eta_{0} & \eta_{0} \neq 0 ; & \\
\operatorname{sign}\left(c_{1}\left[1-\eta_{1}\right]\right) \infty & \eta_{0}=0 ; & \eta_{1} \neq 1 ; & \\
0 & \eta_{0}=0 ; & \eta_{1}=1 ; & \eta_{2}>2 ; \\
-2 c_{2} c_{0} / c_{1}^{2} & \eta_{0}=0 ; & \eta_{1}=1 ; & \eta_{2}=2 ; \\
-\operatorname{sign}\left(c_{2}\right) \infty & \eta_{0}=0 ; & \eta_{1}=1 ; & \eta_{2} \in(1,2) .
\end{array}\right.
$$

Therefore, the deceleration parameter can be either finite or infinite, in particular, it has an infinite limit only for a certain subset of the sudden singularities $\eta_{0}=0$, and for bangs and crunches the limit is always finite. This is largely because the definition of the deceleration parameter was carefully chosen to eliminate the leading $t$ behaviour whenever possible.

\section{Polynomial curvature singularities}

Now to decide if the cosmological milestones we have defined are curvature singularities, we need to have a look at the Riemann tensor. The curvature is measured by the Riemann tensor from which can be constructed various scalar quantities, such as the Ricci scalar $R$, or higher order scalars $R^{a b} R_{a b}, R^{a b c d} R_{a b c d}$, etc... If any of those 
scalars go to infinity when approaching some point $x$, there is a polynomial curvature singularity at this point $x$.

Because of the symmetries of FRW geometry, the Weyl tensor is automatically zero and so it suffices to consider the Ricci tensor (and implicitly the Ricci scalar). But because of spherical symmetry, and the perhaps less obvious translational symmetry, the only two non-zero orthonormal independent components of the Ricci tensor are

$$
R_{\hat{t} \hat{t}} \quad \text { and } \quad R_{\hat{r} \hat{r}}=R_{\hat{\theta} \hat{\theta}}=R_{\hat{\phi} \hat{\phi}} .
$$

Thus to test for all possible polynomial curvature singularities it suffices to test for singularities in, for instance, $R_{\hat{t} \hat{t}}$ and $R_{\hat{r} \hat{r}}$. Alternatively one could consider $R_{\hat{t} \hat{t}}$ and the Ricci scalar $R$, or even $R_{\hat{t} \hat{t}}$ and $G_{\hat{t} \hat{t}}$.

From the metric 3.1, we can calculate the $R_{\hat{t} \hat{t}}$ and $G_{\hat{t} \hat{t}}$ components in an orthonormal basis. We find:

$$
\begin{gathered}
R_{\hat{t} \hat{t}}=-3 \frac{\ddot{a}}{a} \\
G_{\hat{t} \hat{t}}=3\left(\frac{\dot{a}^{2}}{a^{2}}+\frac{k}{a^{2}}\right) .
\end{gathered}
$$

The interest of using those particular two combinations of the orthonormal components of the Ricci tensor is that they satisfy interesting properties:

- they are linearly independent;

- $R_{\hat{t} \hat{t}}$ is independent of the curvature of space (no $k$ dependence);

- $G_{\hat{t} \hat{t}}$ is independent of $\ddot{a}$ (minimizing the number of derivatives involved);

- testing these two objects for finiteness is sufficient to completely characterize all polynomial curvature singularities in a FRW geometry.

Theorem 7. The $R_{\hat{t} \hat{t}}$ theorem:

Consider a generalized power series expansion of the scale factor as defined in Definition 1, the $R_{\hat{t} \hat{t}}$ component of the Ricci tensor in an orthonormal basis remains finite, as the cosmological milestone is approached, only provided:

- $\eta_{0}=0, \eta_{1} \geq 2$; 
- $\eta_{0}=0, \eta_{1}=1, \eta_{2} \geq 2$;

- $\eta_{0}=1$ and $\eta_{1} \geq 3$.

That is, except for these above rather limited cases, $R_{\hat{t} \hat{t}}$ will blow up to infinity as the cosmological milestone is approached.

Proof. The $R_{\hat{t} \hat{t}}$ component of the Ricci tensor can be written as

$$
R_{\hat{t} \hat{t}}=-3 \frac{\ddot{a}}{a} .
$$

Using the generalized power series expansion we can rewrite the $R_{\hat{t} \hat{t}}$ term only as a function of some $\left(t-t_{\odot}\right)$ factors in the vicinity of the event.

Then provided $\eta_{0} \neq 0$ and $\eta_{0} \neq 1$

$$
R_{\hat{t} \hat{t}}=-3 \frac{\ddot{a}}{a} \sim-3 \frac{\eta_{0}\left(\eta_{0}-1\right)}{\left(t-t_{\odot}\right)^{2}} .
$$

If $\eta_{0}=0$, then provided $\eta_{1} \neq 1$

$$
R_{\hat{t} \hat{t}}=-3 \frac{\ddot{a}}{a} \sim-3 \frac{\eta_{1}\left(\eta_{1}-1\right) c_{1}}{c_{0}}\left(t-t_{\odot}\right)^{\eta_{1}-2} .
$$

If both $\eta_{0}=0$ and $\eta_{1}=1$

$$
R_{\hat{t} \hat{t}}=-3 \frac{\ddot{a}}{a} \sim-3 \frac{\eta_{2}\left(\eta_{2}-1\right) c_{2}}{c_{0}}\left(t-t_{\odot}\right)^{\eta_{2}-2} .
$$

Finally, if $\eta_{0}=1$, then ( since $\eta_{1}>1$ )

$$
R_{\hat{t} \hat{t}}=-3 \frac{\ddot{a}}{a} \sim-3 \frac{\eta_{1}\left(\eta_{1}-1\right) c_{1}}{c_{0}}\left(t-t_{\odot}\right)^{\eta_{1}-3} .
$$

The generic result is

$$
\lim _{t \rightarrow t_{\odot}} R_{\hat{t} \hat{t}}=\operatorname{sign}\left(\eta_{0}\left[1-\eta_{0}\right]\right) \infty \quad \text { for } \quad \eta_{0} \neq 0 ; \quad \eta_{0} \neq 1,
$$

For the "exceptional" cases we get:

- If $\eta_{0}=0$, then provided $\eta_{1} \neq 1$

$$
\lim _{t \rightarrow t_{\odot}} R_{\hat{t} \hat{t}}=\left\{\begin{array}{lll}
0 & \eta_{0}=0 ; & \eta_{1}>2 ; \\
-6 c_{1} / c_{0} & \eta_{0}=0 ; & \eta_{1}=2 ; \\
\operatorname{sign}\left(c_{1}\right) \infty & \eta_{0}=0 ; & \eta_{1} \in(1,2) ; \\
-\operatorname{sign}\left(c_{1}\right) \infty & \eta_{0}=0 ; & \eta_{1} \in(0,1) ;
\end{array}\right.
$$


- If both $\eta_{0}=0$ and $\eta_{1}=1$

$$
\lim _{t \rightarrow t_{\odot}} R_{\hat{t} \hat{t}}=\left\{\begin{array}{llll}
0 & \eta_{0}=0 ; & \eta_{1}=1 ; & \eta_{2}>2 ; \\
-6 c_{2} / c_{0} & \eta_{0}=0 ; & \eta_{1}=1 ; & \eta_{2}=2 ; \\
-\operatorname{sign}\left(c_{2}\right) \infty & \eta_{0}=0 ; & \eta_{1}=1 ; & \eta_{2} \in(1,2) ;
\end{array}\right.
$$

- Finally, if $\eta_{0}=1$, then (since $\eta_{1}>1$ )

$$
\lim _{t \rightarrow t_{\odot}} R_{\hat{t} \hat{t}}=\left\{\begin{array}{lll}
0 & \eta_{0}=1 ; & \eta_{1}>3 ; \\
-18 c_{1} / c_{0} & \eta_{0}=1 ; & \eta_{1}=3 ; \\
-\operatorname{sign}\left(c_{1}\right) \infty & \eta_{0}=1 ; & \eta_{1} \in(0,3) .
\end{array}\right.
$$

To summarize, in the vicinity of the cosmological milestone, we can write

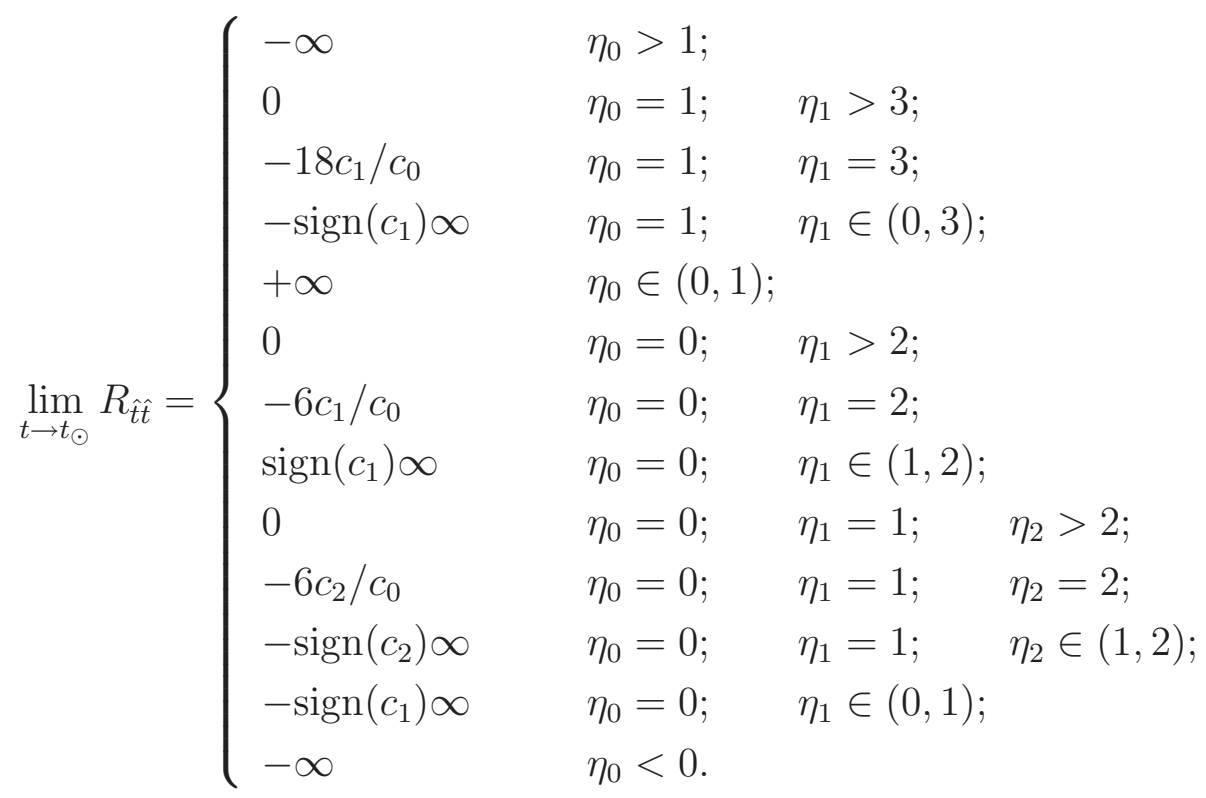

Therefore $R_{\hat{t} \hat{t}}$ is finite provided

- $\eta_{0}=0, \eta_{1} \geq 2$

- $\eta_{0}=0, \eta_{1}=1, \eta_{2} \geq 2$;

- $\eta_{0}=1$ and $\eta_{1} \geq 3$. 
Theorem 8. The $G_{\hat{t} \hat{t}}$ theorem:

Consider a generalized power series expansion of the scale factor as defined in Definition 1, the $G_{\hat{t} \hat{t}}$ component of the Ricci tensor in an orthonormal basis remains finite, as the cosmological milestone is approached, only provided:

$$
\begin{array}{ll}
\eta_{0}=0 & \eta_{1} \geq 1 \\
\eta_{0}=1 & \eta_{1} \geq 3 \quad \text { and } \quad c_{0}^{2}+k=0 .
\end{array}
$$

(Note that $c_{0}^{2}+k=0$ implies $k=-1$, and $c_{0}=1$.)

That is, except for these above rather limited cases, $G_{\hat{t} \hat{t}}$ will blow up to infinity as the cosmological milestone is approached.

Proof. The $G_{\hat{t} \hat{t}}$ term can be written as a function of the scale factor, its first derivative and the curvature parameter $k$,

$$
G_{\hat{t} \hat{t}}=3\left(\frac{\dot{a}^{2}}{a^{2}}+\frac{k}{a^{2}}\right)=3\left(H^{2}+\frac{k}{a^{2}}\right) .
$$

To analyze $G_{\hat{t} \hat{t}}$ recall that the condition that the Hubble parameter $H$ remain finite was $\eta_{0}=0, \eta_{1} \geq 1$ from Theorem 5 . In the vicinity of the milestone, we have

$$
G_{\hat{t} \hat{t}} \sim 3\left[\frac{\eta_{0}^{2}}{\left(t-t_{\odot}\right)^{2}}+\frac{k}{c_{0}^{2}\left(t-t_{\odot}\right)^{2 \eta_{0}}}\right] \quad \eta_{0} \neq 0
$$

while

$$
G_{\hat{t} \hat{t}} \sim 3\left[\frac{\eta_{1}^{2} c_{1}^{2}\left(t-t_{\odot}\right)^{2\left(\eta_{1}-1\right)}+k}{c_{0}^{2}}\right] \quad \eta_{0}=0 .
$$

Whenever $\eta_{0}>1$, the term $k / c_{0}^{2}\left(t-t_{\odot}\right)^{2 \eta_{0}}$ with $k \neq 0$ is dominant and it tends to $\infty$. In the case of $\eta_{0}>1$ and $k=0$, the term $\eta_{0}^{2} /\left(t-t_{\odot}\right)^{2}$ is dominant and it tends to $\infty$. Whenever $\eta_{0}<1$ and $\eta_{0} \neq 0$, the term $\eta_{0}^{2} /\left(t-t_{\odot}\right)^{2}$ is dominant and therefore, $G_{\hat{t} \hat{t}} \rightarrow \infty$. For the special case where $\eta_{0}=1, G_{\hat{t} \hat{t}}$ is a difference of squares and one term can be balanced against the other. Finally, when $\eta_{0}=0$ the first term needs to be developed further using the indicial exponent $\eta_{1}$. 
Therefore, after further calculations, we find,

$$
\lim _{t \rightarrow t_{\odot}} G_{\hat{t} \hat{t}}=\left\{\begin{array}{llll}
\operatorname{sign}(k) \infty & \eta_{0}>1 ; & k \neq 0 \\
+\infty & \eta_{0}>1 ; & k=0 ; \\
\operatorname{sign}\left(c_{0}^{2}+k\right) \infty & \eta_{0}=1 ; & c_{0}^{2}+k \neq 0 ; & \\
0 & \eta_{0}=1 ; & c_{0}^{2}+k=0 ; & \eta_{1}>3 ; \\
18 c_{1} & \eta_{0}=1 ; & c_{0}^{2}+k=0 ; & \eta_{1}=3 ; \\
\operatorname{sign}\left(c_{1}\right) \infty & \eta_{0}=1 ; & c_{0}^{2}+k=0 ; & \eta_{1} \in(1,3) \\
+\infty & \eta_{0} \in(0,1) ; & \\
3 k / c_{0}^{2} & \eta_{0}=0 ; & \eta_{1}>1 ; \\
3\left(c_{1}^{2}+k\right) / c_{0}^{2} & \eta_{0}=0 ; & \eta_{1}=1 \\
+\infty & \eta_{0}=0 ; & \eta_{1} \in(0,1) .
\end{array}\right.
$$

Thus the necessary and sufficient conditions for $G_{\hat{t} \hat{t}}$ to remain finite at the cosmological milestone are:

- $\eta_{0}=0, \eta_{1} \geq 1$

- $\eta_{0}=1, c_{0}^{2}+k=0$, and $\eta_{1} \geq 3$.

(Note that $c_{0}^{2}+k=0$ implies $k=-1$, and $c_{0}=1$.)

Theorem 9. Polynomial curvature singularity theorem:

Consider a generalized power series expansion of the scale factor as defined in Definition 1, every cosmological milestone is a polynomial curvature singularity at the event except for the rather limited classes of cosmological milestones that satisfy the necessary and sufficient conditions:

- $\eta_{0}=0 \quad \eta_{1} \geq 2$ or;

- $\eta_{0}=0 \quad \eta_{1}=1 \quad \eta_{2} \geq 2$ or;

- $\eta_{0}=1 \quad \eta_{1} \geq 3 \quad$ and $\quad k=-1, c_{0}=1$.

That is, for a particular sub-class of sudden singularities $\left(\eta_{0}=0\right)$ and for a rather exceptional type of big bang/crunch (as detailed above), there is no polynomial curvature singularity. 
Proof. From Theorem 7 and 8, we have determined the conditions for $R_{\hat{t} \hat{t}}$ and $G_{\hat{t} \hat{t}}$ (respectively) to remain finite at the time the cosmological milestone occurs.

For $R_{\hat{t} \hat{t}}$, those conditions are

- $\eta_{0}=0, \eta_{1} \geq 2$;

- $\eta_{0}=0, \eta_{1}=1, \eta_{2} \geq 2$;

- $\eta_{0}=1$ and $\eta_{1} \geq 3$.

For $G_{\hat{t} \hat{t}}$, those conditions are

- $\eta_{0}=0, \eta_{1} \geq 1$;

- $\eta_{0}=1, c_{0}^{2}+k=0$, and $\eta_{1} \geq 3$.

(Note that $c_{0}^{2}+k=0$ implies $k=-1$, and $c_{0}=1$.)

Combining those two results, the necessary and sufficient conditions for both $R_{\hat{t} \hat{t}}$ and $G_{\hat{t} \hat{t}}$ to remain finite, so that a cosmological milestone is not a polynomial curvature singularity, are:

- $\eta_{0}=0, \eta_{1} \geq 2$;

- $\eta_{0}=0, \eta_{1}=1, \eta_{2} \geq 2$;

- $\eta_{0}=1, k=-1, c_{0}=1$, and $\eta_{1} \geq 3$.

Note that the case $\eta_{0}=1$ that corresponds to an exceptional type of big bang/crunch is asymptotic to a Milne universe. Indeed, the special case $a(t)=t, k=-1$ is called the Milne universe and is actually a disguised portion of Minkowski space. It corresponds to the interior of the future light cone based at some randomly specified point in Minkowski space, with a spatial foliation defined by the proper time hyperboloids based on that event. Since this spacetime is flat, it corresponds to a universe which on the largest scales is empty. This is not a popular cosmological model.

Any other conditions that are not mentioned above imply that $R_{\hat{t} \hat{t}}$ and/or $G_{\hat{t} \hat{t}}$ blow up at the time of the event; therefore, all other cases imply a polynomial curvature singularity. 
Even if there are a few classes of cosmological milestones that are not polynomial singularities, one can wonder what happens to them when looking at finite-order derivatives of the curvature tensor. Is it still possible to find an interesting type of singularity where the derivative curvature singularity does not blow up?

\section{Derivative curvature singularities}

Definition 9. A derivative curvature singularity is defined by some polynomial constructed from finite-order derivatives of the curvature tensor blowing up.

In our case, because of the symmetries of the FRW universe the only interesting derivatives will be time derivatives, and so the only objects we need to consider are

$$
\frac{\mathrm{d}^{n} R_{\hat{t} \hat{t}}}{\mathrm{~d}^{n} t} \quad \text { and } \quad \frac{\mathrm{d}^{n} G_{\hat{t} \hat{t}}}{\mathrm{~d}^{n} t} .
$$

Theorem 10. Derivative curvature singularity theorem

The only two situations in which a cosmological milestone is not a derivative curvature singularity are if:

- $\eta_{0}=0, \eta_{i} \in Z^{+}$; corresponding to an extremality event (bounce, turnaround, or inflexion event) rather than a bang, crunch, rip, or sudden singularity;

- $\eta_{0}=1, k=-1, c_{0}=1, \eta_{i} \in Z^{+}$, and $\eta_{1} \geq 3$; corresponding to a FRW geometry that smoothly asymptotes near the cosmological milestone to the Riemann-flat Milne universe.

Proof. We can write $\mathrm{d}^{n} R_{\hat{t} \hat{t}} / \mathrm{d}^{n} t$ and $\mathrm{d}^{n} G_{\hat{t} \hat{t}} / \mathrm{d}^{n} t$ using the scale factor $a(t)$ and its derivatives $a^{(n)}(t)$,

$$
\frac{\mathrm{d}^{n} R_{\hat{t} \hat{t}}}{\mathrm{~d}^{n} t}=-3 \frac{a^{(n+2)}}{a}+\text { (lower-order derivatives) }
$$

while

$$
\frac{\mathrm{d}^{n} G_{\hat{t} \hat{t}}}{\mathrm{~d}^{n} t}=3 \frac{\dot{a} a^{(n+1)}}{a^{2}}+\text { (lower-order derivatives). }
$$

Thus to avoid a $n$ th-order derivative curvature singularity we must at the very least keep $a^{(n+2)} / a$ finite, and furthermore all related lower-order derivatives of the 
form $a^{(j)} / a$, with $j \leq n+2$, must also be finite. To prevent any arbitrary-order derivative singularity from occurring, that is for both $\mathrm{d}^{n} R_{\hat{t} \hat{t}} / \mathrm{d}^{n} t$ and $\mathrm{d}^{n} G_{\hat{t} \hat{t}} / \mathrm{d}^{n} t$ to remain finite for all $n \in Z^{+}$, we must force all $a^{(j)} / a$ to remain finite. This condition holds in addition to the constraint coming from polynomial curvature singularities derived above.

The first constraint to hold, so that there is no polynomial curvature singularity, is $\eta_{0}=0$. In this case, the scale factor can be written as:

$$
a(t)=c_{0}+c_{1}\left|t-t_{\odot}\right|^{\eta_{1}}+c_{2}\left|t-t_{\odot}\right|^{\eta_{2}}+c_{3}\left|t-t_{\odot}\right|^{\eta_{3}}+\ldots
$$

Remember that the dominant term in the scale factor is $c_{0}$ which implies that $a(t)$ finite at the time of the event. Therefore, for $a^{(j)} / a$ to remain finite it suffices that $a^{(j)}$ be finite.

The second constraint to hold, so that there is no polynomial curvature singularity, is $\eta_{0}=1$. In this case, the scale factor can be written as:

$$
a(t)=c_{0}\left|t-t_{\odot}\right|+c_{1}\left|t-t_{\odot}\right|^{\eta_{1}}+c_{2}\left|t-t_{\odot}\right|^{\eta_{2}}+c_{3}\left|t-t_{\odot}\right|^{\eta_{3}}+\ldots
$$

Remember that the dominant term in the scale factor is $c_{0}\left|t-t_{\odot}\right|$ which implies that $a(t)$ is not finite at the time of the event. However, the term $\left|t-t_{\odot}\right|$ from $a(t)$ can be "absorbed" in the term $a^{(n+2)}$ as follows:

$$
\frac{a^{(j)}}{a}=\frac{\sum_{i} c_{i} \eta_{i} \ldots\left(\eta_{i}-j+1\right)\left|t-t_{\odot}\right|^{\eta_{i}-j}}{c_{0}\left|t-t_{\odot}\right|+c_{1}\left|t-t_{\odot}\right|^{\eta_{1}}+c_{2}\left|t-t_{\odot}\right|^{\eta_{2}}+c_{3}\left|t-t_{\odot}\right|^{\eta_{3}}+\ldots},
$$

keeping only dominant terms, we have

$$
\frac{a^{(j)}}{a} \sim \frac{\sum_{i} c_{i} \eta_{i} \ldots\left(\eta_{i}-j+1\right)\left|t-t_{\odot}\right|^{\eta_{i}-j}}{c_{0}\left|t-t_{\odot}\right|} \sim \frac{\sum_{i} c_{i} \eta_{i} \ldots\left(\eta_{i}-j+1\right)\left|t-t_{\odot}\right|^{\eta_{i}-j-1}}{c_{0}}
$$

Therefore, for $a^{(j)} / a$ to remain finite it suffices that $a^{(j)}$ be finite.

These two constraints also imply that to force all $a^{(j)}$ to remain finite, we must force all the indicial exponents $\eta_{i}$ to be non-negative integers, thus making $a(t)$ a Taylor series. Indeed, we have

$$
a^{(j)}=\sum_{i} c_{i} \eta_{i} \ldots\left(\eta_{i}-j+1\right)\left|t-t_{\odot}\right|^{\eta_{i}-j}
$$


and $a^{(j)}$ is finite if and only if

$$
\left|t-t_{\odot}\right|^{\eta_{i}-j} \longrightarrow \text { finite }
$$

that is,

$$
\forall i \quad \eta_{i}-j \geqslant 0 \quad \Rightarrow \quad \eta_{i} \geqslant j
$$

Thus, all $\eta_{i}$ must be positive or null. They must be integers as well for $a^{(j)}$ to remain finite: otherwise, there will be eventually a value of $j$ that will make the term $\eta_{i}-j<0$, to avoid that $\eta_{i}$ must be integers so the term $\left|t-t_{\odot}\right|^{\eta_{i}-j}$ disappear and do not tend to infinity.

Hence, we have demonstrated that almost all cosmological milestones are physical singularities, apart from a very limited sub-class corresponding to either extremality events (bounces, turnarounds, inflexion events) or an asymptotically empty universe.

\subsection{Cosmological milestones and dynamics.}

In the previous section, we have considered only kinematics, from now on, we will use the Friedmann equations and the Einstein equations to take our definitions to a dynamic level.

\subsubsection{Introduction}

To now start to include dynamics we relate the geometry to the density and pressure using the Friedmann equations and then ask what happens to the energy conditions at the cosmological milestones. Even though we may have good reason to suspect that the energy conditions are not truly fundamental [3]: they make a very good first pass at the problem of quantifying just how "strange" physics gets at the cosmological milestone. 
Using the Friedmann equations, we can write the pressure $p(t)$ and the density $\rho(t)$ using the scale factor and its first two derivatives:

$$
\begin{aligned}
\rho(t) & =3\left(\frac{\dot{a}^{2}}{a^{2}}+\frac{k}{a^{2}}\right), \\
p(t) & =-2 \frac{\ddot{a}}{a}-\frac{\dot{a}^{2}}{a^{2}}-\frac{k}{a^{2}}, \\
\rho(t)+3 p(t) & =-6 \frac{\ddot{a}}{a} .
\end{aligned}
$$

Therefore, we have

$$
\rho+p=2\left(-\frac{\ddot{a}}{a}+\frac{\dot{a}^{2}}{a^{2}}+\frac{k}{a^{2}}\right)
$$

and

$$
\rho-p=2\left(\frac{\ddot{a}}{a}+2 \frac{\dot{a}^{2}}{a^{2}}+2 \frac{k}{a^{2}}\right) .
$$

The standard energy conditions are the null, weak, strong, and dominant energy conditions ${ }^{6}$ which for a FRW spacetime specialise to $[47,62,3]$ :

- $[\mathrm{NEC}] \rho+p \geq 0$.

In view of equation (3.118) this reduces to

$$
\ddot{a} \leq \frac{\dot{a}^{2}+k}{a} ; \quad \text { that is } \quad k \geq a \ddot{a}-\dot{a}^{2} .
$$

- $[$ WEC] This specializes to the NEC plus $\rho \geq 0$.

This reduces to the NEC plus the condition

$$
\dot{a}^{2}+k \geq 0 ; \quad \text { that is } \quad k \geq-\dot{a}^{2} .
$$

- $[\mathrm{SEC}]$ This specializes to the NEC plus $\rho+3 p \geq 0$.

This reduces to the NEC plus the deceleration condition

$$
\ddot{a} \leq 0 \text {. }
$$

\footnotetext{
${ }^{6}$ See section 2.6 for more details on the energy conditions.
} 
- $[\mathrm{DEC}] \rho \pm p \geq 0$.

This reduces to the NEC plus the condition

$$
\ddot{a} \geq-\frac{2\left(\dot{a}^{2}+k\right)}{a} ; \quad \text { that is } \quad k \geq-\frac{\left(a \ddot{a}+2 \dot{a}^{2}\right)}{2} .
$$

The null energy condition NEC is the most interesting condition because it is the weakest of the standard energy conditions and it leads to the strongest theorems.

\subsubsection{NEC}

We have seen that the NEC is satisfied iff $k \geqslant a \ddot{a}-\dot{a}^{2}$.

Theorem 11. The NEC is definitely satisfied (meaning the inequality is strict) at a generic cosmological milestone iff:

- Big bangs/crunches:

$-\eta_{0}>1, k=+1$

- $\eta_{0}=1:$ for $k=+1$ and $k=0$, and also for $k=-1$ with the proviso that $c_{0}>1$;

$-\eta_{0} \in(0,1):$ for any value of $k$;

- Sudden singularities/extremality events:

$-\eta_{0}=0$ subject to the additional constraints:

$* \eta_{1}>2$ and $k=+1$;

$* \eta_{1}=2$ and $k>2 c_{0} c_{1}$;

$* \eta_{1} \in(1,2)$ and $c_{1}<0$;

$* \eta_{1}=1, \eta_{2}>2$ : for $k=+1, k=0$, and for $k=-1$ with the proviso $\left|c_{1}\right|>1$;

$* \eta_{1}=1, \eta_{2}=2:$ for $k>2 c_{0} c_{2}-c_{1}^{2}$;

$* \eta_{1}=1, \eta_{2} \in(1,2)$, and $c_{2}<0$;

$* \eta_{1} \in(0,1)$, and $c_{1}>0$. 
Theorem 12. The NEC is marginally satisfied (meaning the non-strict inequality is actually an equality) at a generic cosmological milestone iff:

- Big bangs/crunches:

$-\eta_{0}>1, k=0$

$-\eta_{0}=1, k=-1$ with the proviso that $c_{0}=1$;

- Sudden singularities/extremality events:

$-\eta_{0}=0$ subject to the additional constraints:

* $\eta_{1}>2$ and $k=0$;

$* \eta_{1}=2$ and $k=2 c_{0} c_{1}$ (which requires $k \neq 0$ );

$* \eta_{1}=1, \eta_{2}>2:$ for $k=-1$ and $c_{1}= \pm 1$;

$* \eta_{1}=1, \eta_{2}=2$, and $k=2 c_{0} c_{2}-c_{1}^{2}$.

Theorem 13. The NEC is definitely violated (the inequality is strictly violated) at a generic milestone iff:

- Big bangs/crunches:

$-\eta_{0}>1, k=-1$

$-\eta_{0}=1:$ for $k=-1$ with the proviso that $c_{0}<1$;

- Sudden singularities/extremality events:

$-\eta_{0}=0$ subject to the additional constraints:

$* \eta_{1}>2$ and $k=-1$;

$* \eta_{1}=2$ and $k<2 c_{0} c_{1}$;

* $\eta_{1} \in(1,2)$ and $c_{1}<0$;

$* \eta_{1}=1, \eta_{2}>2$ : for $k=-1$ with the proviso $\left|c_{1}\right|<1$;

$* \eta_{1}=1, \eta_{2}=2$ : for $k<2 c_{0} c_{2}-c_{1}^{2}$;

$* \eta_{1}=1, \eta_{2} \in(1,2)$, and $c_{2}>0$; 


$$
* \eta_{1} \in(0,1) \text {, and } c_{1}<0 \text {. }
$$

- Big rips:

$$
-\eta_{0}<0 .
$$

In particular, "big rips" $\left(\eta_{0}<0\right)$ will violate the NEC in the vicinity of the milestone and therefore, they will also violate every other energy conditions.

Proof. The NEC is satisfied if $k \geqslant a \ddot{a}-\dot{a}^{2}$. Remember that near the cosmological milestone we can write the scale factor as a generalized power series as defined in Defintion 1:

$$
a(t)=c_{0}\left|t-t_{\odot}\right|^{\eta_{0}}+c_{1}\left|t-t_{\odot}\right|^{\eta_{1}}+c_{2}\left|t-t_{\odot}\right|^{\eta_{2}}+c_{3}\left|t-t_{\odot}\right|^{\eta_{3}}+\ldots
$$

Therefore, near any generic cosmological milestone

$$
a \ddot{a}-\dot{a}^{2} \sim-\eta_{0} c_{0}^{2} t^{2\left(\eta_{0}-1\right)} ; \quad\left(\eta_{0} \neq 0\right),
$$

while in the degenerate cases

$$
a \ddot{a}-\dot{a}^{2} \sim c_{0} c_{1} \eta_{1}\left(\eta_{1}-1\right)\left(t-t_{\odot}\right)^{\left(\eta_{1}-2\right)} ; \quad\left(\eta_{0}=0 ; \eta_{1} \neq 1\right),
$$

and

$$
a \ddot{a}-\dot{a}^{2} \sim c_{0} c_{2} \eta_{2}\left(\eta_{2}-1\right)\left(t-t_{\odot}\right)^{\left(\eta_{2}-2\right)}-c_{1}^{2} ; \quad\left(\eta_{0}=0 ; \eta_{1}=1\right) .
$$

After further calculations, we find the limit of $\left(a \ddot{a}-\dot{a}^{2}\right)$ when the milestone occurs:

$$
\lim _{t \rightarrow t_{\odot}}\left(a \ddot{a}-\dot{a}^{2}\right)=\left\{\begin{array}{llll}
0 & \eta_{0}>1 ; & & \\
-c_{0}^{2} & \eta_{0}=1 ; & & \\
-\infty & \eta_{0} \in(0,1) ; & & \\
0 & \eta_{0}=0 ; & \eta_{1}>2 ; \\
2 c_{0} c_{1} & \eta_{0}=0 ; \quad \eta_{1}=2 ; & \\
+\operatorname{sign}\left(c_{1}\right) \infty & \eta_{0}=0 ; \quad \eta_{1} \in(1,2) & \\
-c_{1}^{2} & \eta_{0}=0 ; & \eta_{1}=1 ; \quad \eta_{2}>2 ; \\
2 c_{0} c_{2}-c_{1}^{2} & \eta_{0}=0 ; & \eta_{1}=1 ; \quad \eta_{2}=2 ; \\
+\operatorname{sign}\left(c_{2}\right) \infty & \eta_{0}=0 ; & \eta_{1}=1 ; \quad \eta_{2} \in(1,2) ; \\
-\operatorname{sign}\left(c_{1}\right) \infty & \eta_{0}=0 ; & \eta_{1} \in(0,1) & \\
+\infty & \eta_{0}<0 . & &
\end{array}\right.
$$


The NEC is definitely satisfied iff:

$$
k>a \ddot{a}-\dot{a}^{2},
$$

that is, iff:

- $\eta_{0}>1, k=+1$;

- $\eta_{0}=1$ : for $k=+1$ and $k=0$, and also for $k=-1$ with the proviso that $c_{0}>1$;

- $\eta_{0} \in(0,1)$ : for any value of $k$;

- $\eta_{0}=0$ subject to the additional constraints:

- $\eta_{1}>2$ and $k=+1$

$-\eta_{1}=2$ and $k>2 c_{0} c_{1}$

$-\eta_{1} \in(1,2)$ and $c_{1}<0$

$-\eta_{1}=1, \eta_{2}>2$ : for $k=+1, k=0$, and for $k=-1$ with the proviso $\left|c_{1}\right|>1$;

$-\eta_{1}=1, \eta_{2}=2$ : for $k>2 c_{0} c_{2}-c_{1}^{2}$;

$-\eta_{1}=1, \eta_{2} \in(1,2)$, and $c_{2}<0$;

$-\eta_{1} \in(0,1)$, and $c_{1}>0$.

This proves Theorem 11 .

The NEC is marginally satisfied iff:

$$
k=a \ddot{a}-\dot{a}^{2},
$$

that is, iff

- $\eta_{0}>1, k=0$

- $\eta_{0}=1, k=-1$ with the proviso that $c_{0}=1$;

- $\eta_{0}=0$ subject to the additional constraints:

$-\eta_{1}>2$ and $k=0$; 


$$
\begin{aligned}
& -\eta_{1}=2 \text { and } k=2 c_{0} c_{1}(\text { which requires } k \neq 0) ; \\
& -\eta_{1}=1, \eta_{2}>2: \text { for } k=-1 \text { and } c_{1}= \pm 1 ; \\
& -\eta_{1}=1, \eta_{2}=2, \text { and } k=2 c_{0} c_{2}-c_{1}^{2} .
\end{aligned}
$$

This proves Theorem 12 .

The NEC is definitely violated iff:

$$
k<a \ddot{a}-\dot{a}^{2},
$$

that is, iff

- $\eta_{0}>1, k=-1$

- $\eta_{0}=1$ : for $k=-1$ with the proviso that $c_{0}<1$;

- $\eta_{0}=0$ subject to the additional constraints:

- $\eta_{1}>2$ and $k=-1$

$-\eta_{1}=2$ and $k<2 c_{0} c_{1}$

$-\eta_{1} \in(1,2)$ and $c_{1}<0$;

- $\eta_{1}=1, \eta_{2}>2$ : for $k=-1$ with the proviso $\left|c_{1}\right|<1$;

$-\eta_{1}=1, \eta_{2}=2$ : for $k<2 c_{0} c_{2}-c_{1}^{2}$;

- $\eta_{1}=1, \eta_{2} \in(1,2)$, and $c_{2}>0$;

$-\eta_{1} \in(0,1)$, and $c_{1}<0$.

- $\eta_{0}<0$.

This proves Theorem 13.

Theorems 11, 12, and 13, present a pattern that is in agreement with the standard folklore, and is a systematic expression of results that are otherwise scattered throughout the literature. For instance, it is immediate that "big rips" always violate the NEC (and hence all the other energy conditions), and that for big bangs and crunches the range $\eta_{0} \in(0,1)$ is preferred, since it is only in this range that the 
NEC holds independent of the spatial curvature. In particular, if the NEC is to hold independent of the sign of space curvature $k$ all the way down to the singularity, then in the vicinity of the singularity the dominant term in the scale factor $a(t)$ is bounded from both above and below by

$$
c_{0}\left(t-t_{*}\right)<a_{\text {dominant }}(t) \leq c_{0} .
$$

Note that for sufficiently violent big bangs $\left(\eta_{0}>1\right)$ and hyperbolic spatial curvature the NEC is violated: this indicates that "phantom matter" need not always lead to a "big rip" [16]; it might in contrast lead to a particularly violent bang or crunch. It is for $\eta_{0}=0$ (which corresponds to either sudden singularities or extremality events) that the analysis becomes somewhat tedious. Certainly, many (though not all) of the sudden singularities violate the NEC.

\subsubsection{WEC}

We have seen that the WEC is satisfied iff

- the NEC is satisfied, that is if $k \geqslant a \ddot{a}-\dot{a}^{2}$,

- and, $\rho \geqslant 0$, that is if $k \geqslant-\dot{a}^{2}$.

Theorem 14. The WEC is definitely satisfied (meaning the inequality is strict) at a generic cosmological milestone iff the NEC is definitely satisfied (Theorem 11).

Theorem 15. The WEC is marginally satisfied (meaning the non-strict inequality is actually an equality) at a generic cosmological milestone iff the NEC is marginally satisfied (Theorem 12).

Theorem 16. The WEC is definitely violated (meaning the inequality is strict violated) at a generic cosmological milestone for any cases that are not mentioned in Theorems 11 or 12 .

Proof. The WEC is satisfied if $k \geqslant a \ddot{a}-\dot{a}^{2}$ and $k \geqslant-\dot{a}^{2}$. The first condition $k \geqslant a \ddot{a}-\dot{a}^{2}$ has been dealt with in the proof of Theorems 11, 12 and 13. We need to 
look at $k \geqslant-\dot{a}^{2}$. Near the cosmological milestone we can write the scale factor as a generalized power series as defined in Definition 1:

$$
a(t)=c_{0}\left|t-t_{\odot}\right|^{\eta_{0}}+c_{1}\left|t-t_{\odot}\right|^{\eta_{1}}+c_{2}\left|t-t_{\odot}\right|^{\eta_{2}}+c_{3}\left|t-t_{\odot}\right|^{\eta_{3}}+\ldots
$$

Therefore, near any generic cosmological milestone

$$
-\dot{a}^{2} \sim-\eta_{0}^{2} c_{0}^{2}\left(t-t_{\odot}\right)^{2\left(\eta_{0}-1\right)} ; \quad\left(\eta_{0} \neq 0\right),
$$

while in the degenerate case

$$
-\dot{a}^{2} \sim-\eta_{1}^{2} c_{1}^{2}\left(t-t_{\odot}\right)^{2\left(\eta_{1}-1\right)} ; \quad\left(\eta_{0}=0\right)
$$

Therefore

$$
\lim _{t \rightarrow t_{\odot}}\left(-\dot{a}^{2}\right)=\left\{\begin{array}{lll}
0 & \eta_{0}>1 ; & \\
-c_{0}^{2} & \eta_{0}=1 ; & \\
-\infty & \eta_{0} \in(0,1) ; \\
0 & \eta_{0}=0 ; & \eta_{1}>1 ; \\
-c_{1}^{2} & \eta_{0}=0 ; & \eta_{1}=1 ; \\
-\infty & \eta_{0}=0 ; & \eta_{1} \in(0,1) \\
-\infty & \eta_{0}<0 . &
\end{array}\right.
$$

Since we want the condition $k>-\dot{a}^{2}$ to hold in addition to the NEC, it follows that the this condition is equivalent to the NEC for bangs crunches and rips, and that the only changes arise when dealing with $\eta_{0}=0$ (sudden singularities or extremality events), but overall the conditions on the NEC are dominant. Therefore, the WEC holds if and only if the NEC holds.

This proves Theorems 14, 15 and 16.

Certainly, many (though not all) of the sudden singularities violate the WEC.

In particular, if the WEC is to hold independent of the sign of space curvature $k$ all the way down to the singularity, then in the vicinity of the singularity the dominant term in the scale factor $a(t)$ is bounded from both above and below by

$$
c_{0}\left(t-t_{*}\right)<a_{\text {dominant }}(t) \leq c_{0} .
$$




\subsubsection{SEC}

We have seen that the SEC is satisfied iff

- the NEC is satisfied, that is if $k \geqslant a \ddot{a}-\dot{a}^{2}$,

- and, $\rho+3 p \geqslant 0$, that is if $\ddot{a} \leqslant 0$.

Theorem 17. The SEC is definitely satisfied (meaning the inequality is strict) at a generic cosmological milestone iff:

- Big bangs/crunches:

$-\eta_{0}=1:$ for $k=+1$ and $k=0$;

$-\eta_{0} \in(0,1):$ for any value of $k$;

- Sudden singularities/ extremality events:

- $\eta_{0}=0$ subject to the additional constraints:

$* \eta_{1}>2, k=+1$ and $c_{1}<0$;

$* \eta_{1}=2, k>2 c_{0} c_{1}$ and $c_{1}<0$;

$* \eta_{1} \in(1,2)$ and $c_{1}<0$;

$* \eta_{1}=1, \eta_{2}>2$ and $c_{2}<0:$ for $k=+1, k=0$, and for $k=-1$ with the proviso $\left|c_{1}\right|>1$;

$* \eta_{1}=1, \eta_{2}=2$ and $c_{2}<0$ : for $k>2 c_{0} c_{2}-c_{1}^{2}$;

$* \eta_{1}=1, \eta_{2} \in(1,2)$, and $c_{2}<0$;

$* \eta_{1} \in(0,1)$, and $c_{1}>0$.

Theorem 18. The SEC is marginally satisfied (meaning the non-strict inequality is actually an equality) at a generic cosmological milestone iff:

- Big bangs/crunches:

$-\eta_{0}=1, c_{0}=1, c_{1}<0$, and $k=-1$

- Sudden singularities/ extremality events: 
$-\eta_{0}=0$ subject to the additional constraints:

$* \eta_{1}>2, k=0$ and $c_{1}<0$;

$* \eta_{1}=2, k=2 c_{0} c_{1}$ and $c_{1}<0$;

(which requires that $c_{0}=-1 /\left(2 c_{1}\right)$ )

$* \eta_{1}=1, \eta_{2}>2$ and $c_{2}<0, k=-1, c_{1}= \pm 1$ and $c_{2}<0$;

$* \eta_{1}=1, \eta_{2}=2$ and $c_{2}<0$, and $k=2 c_{0} c_{2}-c_{1}^{2}$;

(which requires that $k=-1$ and therefore $2 c_{0} c_{2}-c_{1}^{2}=-1$ )

Theorem 19. The SEC is definitely violated (meaning the inequality is strict violated) at a generic cosmological milestone for any cases that are not mentioned in Theorems 17 or 18 .

Proof. The SEC is satisfied if $k \geqslant a \ddot{a}-\dot{a}^{2}$ and $\ddot{a} \leqslant 0$. The first condition $k \geqslant a \ddot{a}-\dot{a}^{2}$ has been dealt with in the proof of Theorems 11,12 and 13 . We need to look at $\ddot{a} \leqslant 0$. Near the cosmological milestone we can write the scale factor as a generalized power series as defined in Defintion 1:

$$
a(t)=c_{0}\left|t-t_{\odot}\right|^{\eta_{0}}+c_{1}\left|t-t_{\odot}\right|^{\eta_{1}}+c_{2}\left|t-t_{\odot}\right|^{\eta_{2}}+c_{3}\left|t-t_{\odot}\right|^{\eta_{3}}+\ldots
$$

Therefore, near any generic cosmological milestone

$$
\ddot{a} \sim \eta_{0}\left(\eta_{0}-1\right) c_{0} t^{\left(\eta_{0}-2\right)} ; \quad\left(\eta_{0} \neq 0, \quad \eta_{0} \neq 1\right),
$$

while in the degenerate cases

$$
\begin{array}{ll}
\ddot{a} \sim \eta_{1}\left(\eta_{1}-1\right) c_{1}\left(t-t_{\odot}\right)^{\left(\eta_{1}-2\right) ;} & \begin{cases}\eta_{0}=0 ; & \eta_{1} \neq 1 ; \\
\eta_{0}=1 ;\end{cases} \\
\ddot{a} \sim \eta_{2}\left(\eta_{2}-1\right) c_{2}\left(t-t_{\odot}\right)^{\left(\eta_{2}-2\right)} ; & \left(\eta_{0}=0 ; \quad \eta_{1}=1 ;\right),
\end{array}
$$

Therefore

$$
\lim _{t \rightarrow t_{\odot}^{+}}[\operatorname{sign}(\ddot{a})]= \begin{cases}+1 & \eta_{0}>1 \\ \operatorname{sign}\left(c_{1}\right) & \eta_{0}=1 ; \\ -1 & \eta_{0} \in(0,1): \\ \operatorname{sign}\left(\eta_{1}\left[\eta_{1}-1\right] c_{1}\right) & \eta_{0}=0 ; \quad \eta_{1} \neq 1 ; \\ \operatorname{sign}\left(c_{2}\right) & \eta_{0}=0 ; \quad \eta_{1}=1 ; \\ +1 & \eta_{0}<0 .\end{cases}
$$


Since we want the condition $\ddot{a} \leq 0$ to hold in addition to the NEC, it follows that the SEC is definitely satisfied when those conditions below hold:

- $\eta_{0}=1$ : for $k=+1$ and $k=0$;

- $\eta_{0} \in(0,1)$ : for any value of $k$;

- $\eta_{0}=0$ subject to the additional constraints:

$-\eta_{1}>2, k=+1$ and $c_{1}<0$

$-\eta_{1}=2, k>2 c_{0} c_{1}$ and $c_{1}<0$;

$-\eta_{1} \in(1,2)$ and $c_{1}<0$;

$-\eta_{1}=1, \eta_{2}>2$ and $c_{2}<0$ : for $k=+1, k=0$, and for $k=-1$ with the proviso $\left|c_{1}\right|>1$;

$-\eta_{1}=1, \eta_{2}=2$ and $c_{2}<0$ : for $k>2 c_{0} c_{2}-c_{1}^{2}$;

$-\eta_{1}=1, \eta_{2} \in(1,2)$, and $c_{2}<0$;

$-\eta_{1} \in(0,1)$, and $c_{1}>0$.

The SEC is marginally satisfied when:

- $\eta_{0}=1, c_{0}=1, c_{1}<0$, and $k=-1$;

- $\eta_{0}=0$ subject to the additional constraints:

$-\eta_{1}>2, k=0$ and $c_{1}<0$;

$-\eta_{1}=2, k=2 c_{0} c_{1}$ and $c_{1}<0$;

(which requires that $c_{0}=-1 /\left(2 c_{1}\right)$ )

$-\eta_{1}=1, \eta_{2}>2$ and $c_{2}<0, k=-1, c_{1}= \pm 1$ and $c_{2}<0$;

$-\eta_{1}=1, \eta_{2}=2$ and $c_{2}<0$, and $k=2 c_{0} c_{2}-c_{1}^{2}$;

(which requires that $k=-1$ and therefore $2 c_{0} c_{2}-c_{1}^{2}=-1$ )

These conditions prove Theorems 17, 18 and 19 
The SEC is definitely violated for all "rips", and is also definitely violated for all "violent" bangs and crunches with $\eta_{0}>1$. The SEC is definitely satisfied for bangs and crunches in the range $\eta_{0} \in(0,1)$. In particular, if the SEC is to hold independent of the sign of space curvature $k$ all the way down to the singularity, then in the vicinity of the singularity the dominant term in the scale factor $a(t)$ is bounded from both above and below by

$$
c_{0}\left(t-t_{*}\right)<a_{\text {dominant }}(t) \leq c_{0} .
$$

For turnarounds or bounces $\eta_{1}=2 n, n \in Z^{+}$, and the sign of $c_{1}$ governs possible SEC violations: Turnarounds satisfy the SEC while bounces violate the $\mathrm{SEC}^{7}$. For inflexion events, where $\eta_{1}=2 n+1, n \in Z^{+}$, the SEC is violated either just before or just after the inflexion event ${ }^{8}$.

\subsubsection{DEC}

We have seen that the DEC is satisfied iff

- the NEC is satisfied, that is if $k \geqslant a \ddot{a}-\dot{a}^{2}$,

- and, $\rho-p \geqslant 0$, that is if $k \geq-\frac{\left(a \ddot{a}+2 \dot{a}^{2}\right)}{2}$.

Theorem 20. The DECis definitely satisfied (meaning the inequality is strict) at a generic cosmological milestone iff:

- Big bangs/crunches:

$$
\begin{aligned}
-\eta_{0} & >1, k=+1 . \\
-\eta_{0} & =1, k=0,+1, \text { and } k=-1 \text { if } c_{0}>1 . \\
-\eta_{0} & \in(1 / 3,1), \text { for all } k . \\
-\eta_{0} & =1 / 3, \\
* & \eta_{1}>5 / 3, k=+1 .
\end{aligned}
$$

\footnotetext{
${ }^{7}$ See also $[47,62]$.

${ }^{8}$ See also $[47,62]$.
} 
$* \eta_{1}=5 / 3, k>-\frac{14}{9} c_{0} c_{1}$.

$* \eta_{1} \in(1 / 3,5 / 3), c_{1}>0$, for all $k$.

- Sudden singularities/extremality events:

$$
\begin{aligned}
-\eta_{0} & =0 \\
& * \eta_{1}>2, k=+1 . \\
& * \eta_{1}=2, \text { with } k>c_{0} \max \left\{2 c_{1},-c_{1}\right\} . \\
& * \eta_{1}=1, \eta_{2}>2, k=0,+1, \text { and } k=-1 \text { if }\left|c_{1}\right|>1 . \\
& * \eta_{1}=1, \eta_{2}=2, k>c_{0} \max \left\{2 c_{1},-c_{1}\right\}-c_{1}^{2} .
\end{aligned}
$$

Theorem 21. The DEC is marginally satisfied (meaning the non-strict inequality is actually an equality) at a generic cosmological milestone iff:

- Big bangs/crunches:

$$
\begin{aligned}
& -\eta_{0}>1, k=0 . \\
& -\eta_{0}=1, k=-1 \text { if } c_{0}=1 . \\
& -\eta_{0}=1 / 3, \\
& \quad * \eta_{1}>5 / 3, k=0 . \\
& * \eta_{1}=5 / 3, k=-\frac{14}{9} c_{0} c_{1} .
\end{aligned}
$$

- Sudden singularities/extremality events:

$$
\begin{aligned}
-\eta_{0} & =0, \\
& * \eta_{1}>2, k=0 . \\
& * \eta_{1}=2, \text { with } k=c_{0} \max \left\{2 c_{1},-c_{1}\right\} . \\
& * \eta_{1}=1, \eta_{2}>2, k=-1 \text { if }\left|c_{1}\right|=1 . \\
& * \eta_{1}=1, \eta_{2}=2, k=c_{0} \max \left\{2 c_{1},-c_{1}\right\}-c_{1}^{2} .
\end{aligned}
$$

Theorem 22. The DEC is definitely violated (meaning the inequality is strict violated) at a generic cosmological milestone for any cases that are not mentioned in Theorems 20 or 21. 
Proof. The DEC is satisfied if $k \geqslant a \ddot{a}-\dot{a}^{2}$ and $k \geq-\left(a \ddot{a}+2 \dot{a}^{2}\right) / 2$. The first condition $k \geqslant a \ddot{a}-\dot{a}^{2}$ has been dealt with in the proof of Theorems 11, 12 and 13. We need to look at $k \geq-\left(a \ddot{a}+2 \dot{a}^{2}\right) / 2$. Near the cosmological milestone we can write the scale factor as a generalized power series as defined in Defintion 1:

$$
a(t)=c_{0}\left|t-t_{\odot}\right|^{\eta_{0}}+c_{1}\left|t-t_{\odot}\right|^{\eta_{1}}+c_{2}\left|t-t_{\odot}\right|^{\eta_{2}}+c_{3}\left|t-t_{\odot}\right|^{\eta_{3}}+\ldots
$$

Therefore, near any generic cosmological milestone

$$
-\frac{\left(a \ddot{a}+2 \dot{a}^{2}\right)}{2} \sim-\frac{\eta_{0}\left(3 \eta_{0}-1\right) c_{0}^{2}}{2}\left(t-t_{\odot}\right)^{2\left(\eta_{0}-1\right)} ; \quad\left(\eta_{0} \neq 0, \quad \eta_{0} \neq 1 / 3\right),
$$

while in the various degenerate cases

$$
\begin{gathered}
-\frac{\left(a \ddot{a}+2 \dot{a}^{2}\right)}{2} \sim-\frac{\eta_{1}\left(\eta_{1}-1\right) c_{0} c_{1}}{2}\left(t-t_{\odot}\right)^{\left(\eta_{1}-2\right)} ; \quad\left(\eta_{0}=0 ; \quad \eta_{1} \neq 1\right), \\
-\frac{\left(a \ddot{a}+2 \dot{a}^{2}\right)}{2} \sim-\frac{\eta_{2}\left(\eta_{2}-1\right) c_{0} c_{2}}{2}\left(t-t_{\odot}\right)^{\left(\eta_{2}-2\right)}-c_{1}^{2} ; \quad\left(\eta_{0}=0 ; \quad \eta_{1}=1\right), \\
-\frac{\left(a \ddot{a}+2 \dot{a}^{2}\right)}{2} \sim-\frac{\left(3 \eta_{1}+2\right)\left(3 \eta_{1}-1\right) c_{0} c_{1}}{18}\left(t-t_{\odot}\right)^{\left(\eta_{1}-5 / 3\right)} ; \quad\left(\eta_{0}=1 / 3\right) .
\end{gathered}
$$


Therefore

$$
\lim _{t \rightarrow t_{\odot}}\left[-\frac{\left(a \ddot{a}+2 \dot{a}^{2}\right)}{2}\right]=\left\{\begin{array}{lll}
0 & \eta_{0}>1 \\
-c_{0}^{2} & \eta_{0}=1 ; \\
-\infty & \eta_{0} \in(1 / 3,1) ; \\
0 & \eta_{0}=1 / 3 ; \quad \eta_{1}>5 / 3 ; \\
-\frac{14}{9} c_{0} c_{1} & \eta_{0}=1 / 3 ; \quad \eta_{1}=5 / 3 ; \\
-\operatorname{sign}\left(c_{1}\right) \infty & \eta_{0}=1 / 3 ; \quad \eta_{1}<5 / 3 ; \\
+\infty & \eta_{0} \in(0,1 / 3) ; \\
0 & \eta_{0}=0 ; \quad \eta_{1}>2 ; \\
-c_{0} c_{1} & \eta_{0}=0 ; \quad \eta_{1}=2 ; \\
-\operatorname{sign}\left(c_{1}\right) \infty & \eta_{0}=0 ; \quad \eta_{1} \in(1,2) \\
-c_{1}^{2} & \eta_{0}=0 ; \quad \eta_{1}=1 ; \quad \eta_{2}>2 ; \\
-c_{0} c_{2}-c_{1}^{2} & \eta_{0}=0 ; \quad \eta_{1}=1 ; \quad \eta_{2}=2 ; \\
-\operatorname{sign}\left(c_{2}\right) \infty & \eta_{0}=0 ; \quad \eta_{1}=1 ; \quad \eta_{2} \in(1,2) ; \\
+\operatorname{sign}\left(c_{1}\right) \infty & \eta_{0}=0 ; & \eta_{1} \in(0,1) ; \\
-\infty & \eta_{0}<0 . &
\end{array}\right.
$$

Remember that to satisfy the DEC one needs to satisfy the NEC in addition to the constraint coming from the above. Let us define

$$
K=\max \left\{\lim _{t \rightarrow t_{\odot}}\left[a \ddot{a}-\dot{a}^{2}\right], \lim _{t \rightarrow t_{\odot}}\left[-\frac{\left(a \ddot{a}+2 \dot{a}^{2}\right)}{2}\right]\right\} .
$$

Then satisfying the DEC at the cosmological milestone is equivalent to the constraint

$$
k \geq K
$$

- For bangs and crunches we calculate:

$$
K=\left\{\begin{array}{lll}
0 & \eta_{0}>1 ; & \\
-c_{0}^{2} & \eta_{0}=1 ; & \\
-\infty & \eta_{0} \in(1 / 3,1) ; & \\
0 & \eta_{0}=1 / 3 ; & \eta_{1}>5 / 3 ; \\
-\frac{14}{9} c_{0} c_{1} & \eta_{0}=1 / 3 ; & \eta_{1}=5 / 3 ; \\
-\operatorname{sign}\left(c_{1}\right) \infty & \eta_{0}=1 / 3 ; & \eta_{1}<5 / 3 ; \\
+\infty & \eta_{0} \in(0,1 / 3) .
\end{array}\right.
$$


- For rips we have:

$$
K=+\infty \quad \eta_{0}<0 .
$$

Note that rips, because they violate the NEC, always violate the DEC, so the particular constraint derived above is not the controlling feature.

- Finally, for sudden singularities and extremality events:

$$
K=\left\{\begin{array}{llll}
0 & \eta_{0}=0 ; & \eta_{1}>2 ; & \\
c_{0} \max \left\{2 c_{1},-c_{1}\right\} & \eta_{0}=0 ; & \eta_{1}=2 ; & \\
+\infty & \eta_{0}=0 ; & \eta_{1} \in(1,2) & \\
-c_{1}^{2} & \eta_{0}=0 ; & \eta_{1}=1 ; & \eta_{2}>2 ; \\
c_{0} \max \left\{2 c_{1},-c_{1}\right\}-c_{1}^{2} & \eta_{0}=0 ; & \eta_{1}=1 ; & \eta_{2}=2 ; \\
+\infty & \eta_{0}=0 ; & \eta_{1}=1 ; & \eta_{2} \in(1,2) ; \\
+\infty & \eta_{0}=0 ; & \eta_{1} \in(0,1) .
\end{array}\right.
$$

As we have now seen (several times), the $\eta_{0}=0$ case is "special" and requires extra care and delicacy in the analysis - this is the underlying reason why "sudden singularities" are so "fragile", and so dependent on the specific details of the particular model. Indeed there are several classes of cosmological milestone for which the DEC is satisfied. For instance, a complete catalogue of the bangs and crunches for which the DEC is satisfied is:

- $\eta_{0}>1, k=0,+1$.

- $\eta_{0}=1, k=0,+1$, and $k=-1$ if $c_{0} \geq 1$.

- $\eta_{0} \in(1 / 3,1)$, for all $k$.

- $\eta_{0}=1 / 3, \eta_{1}>5 / 3, k=0,+1$.

- $\eta_{0}=1 / 3, \eta_{1}=5 / 3, k \geq-\frac{14}{9} c_{0} c_{1}$.

- $\eta_{0}=1 / 3, \eta_{1} \in(1 / 3,5 / 3), c_{1}>0$, for all $k$.

Similarly, a complete catalogue of the sudden singularities for which the DEC is satisfied is: 
- $\eta_{0}=0, \eta_{1}>2, k=0,+1$.

- $\eta_{0}=0, \eta_{1}=2$, with $k \geq c_{0} \max \left\{2 c_{1},-c_{1}\right\}$.

(In particular this requires $k=+1$ though that is not sufficient.)

- $\eta_{0}=0, \eta_{1}=1, \eta_{2}>2, k=0,+1$, and $k=-1$ if $\left|c_{1}\right| \geq 1$.

- $\eta_{0}=0, \eta_{1}=1, \eta_{2}=2, k \geq c_{0} \max \left\{2 c_{1},-c_{1}\right\}-c_{1}^{2}$.

These catalogues prove Theorem 20, 21, and 22 .

If one wishes to use "normal" matter (that is, matter satisfying all of the energy conditions) to drive a bang or a crunch independent of the sign of space curvature $k$, then one is forced in a model independent manner into the range $\eta_{0} \in(1 / 3,1)$. In particular, if the DEC is to hold independent of the sign of space curvature $k$ all the way down to the singularity, then in the vicinity of the singularity the dominant term in the scale factor $a(t)$ is bounded from both above and below by

$$
c_{0}\left(t-t_{*}\right)<a_{\text {dominant }}(t) \leq c_{0}\left(t-t_{*}\right)^{1 / 3} \text {. }
$$

The fact that we find special cases of sudden singularities that do not violate the DEC appears at first glance, to contradict the analysis of Lake [52], who claimed that all sudden singularities violate the DEC. The resolution of this apparent contradiction lies in the question "just how sudden is the sudden singularity?" Lake takes as his definition $\ddot{a}\left(t \rightarrow t_{\odot}\right)=-\infty$, corresponding in our analysis to $\eta_{0}=0$ with either $\eta_{1} \in(0,1) \cup(1,2)$ or $\eta_{1}=1$ with $\eta_{2}<2$. In this situation our results certainly agree with those of Lake: if $\ddot{a}\left(t \rightarrow t_{\odot}\right)=-\infty$ then the DEC is certainly violated. However, our "counterexamples" where the DEC is satisfied all satisfy $\ddot{a}\left(t \rightarrow t_{\odot}\right)=$ finite, and for these counterexamples it is only some higher derivative $a^{(n)}\left(t \rightarrow t_{\odot}\right)$ for $n \geq 3$ that diverges. Sudden singularities of this type are sufficiently "gentle" that at least for some of them even the DEC can be satisfied all the way to the singularity: a conclusion completely in agreement with Barrow and Tsagas [8], though now we have a complete characterization of those situations for which the DEC can be satisfied. 


\subsection{Total age of the universe}

In this section, we express the density as a function of the scale factor $\rho(a(t))$ and analyze the Friedmann equation (3.2) rewritten with $\rho(a(t))$. This equation can then be formally solved by integration to find $t(a)$, time as a function of the scale parameter.

We adopt a barotropic equation of state by assuming the statement that the pressure is a function of density, $p(\rho)$. If we express the density as a function of the scale factor, we can write $\rho=\rho(a(t))$. We can then write,

$$
\frac{d \rho}{\mathrm{d} a}=\frac{d \rho / d t}{d a / d t}
$$

Now rewriting the conservation equation, in units where $8 \pi G_{N}=1$ and $c=1$, we obtain,

$$
\frac{d \rho}{d a}+3\left[\frac{\rho+p(\rho)}{a}\right]=0
$$

so that

$$
-\frac{d \rho}{3[\rho+p(\rho)]}=\frac{d a}{a}
$$

Integrating, we get

$$
-\int \frac{d \rho}{3[\rho+p(\rho)]}=\int \frac{d a}{a}
$$

Definition 10. Define,

$$
h(\rho)=\int_{\rho_{p=0}}^{\rho} \frac{d \bar{\rho}}{[\bar{\rho}+p(\bar{\rho})]}
$$

where $\rho_{p=0}$ is the density at zero pressure which is defined by

$$
p\left(\rho_{p=0}\right)=0 .
$$

The function $h(\rho)$ is called the relativistic specific enthalpy.

Using Definition 10, then in terms of the present day density $\rho_{0}$ and the present day scale factor $a_{0}$

$$
-\frac{1}{3} h(\rho)-h\left(\rho_{0}\right)=\ln \left(a / a_{0}\right) .
$$


We can now write

$$
a=a_{0} \exp \left[-\frac{1}{3}\left(h(\rho)-h\left(\rho_{0}\right)\right)\right]
$$

and deduce the existence of a function $f$ such as

$$
a=f(\rho) .
$$

Note that when the density increases, assuming the NEC holds so does the enthalpy and therefore, the scale factor decreases. Now, apply the inverse function theorem to the function $f$ in equation (3.164). After all

$$
\frac{d h}{d \rho}=\frac{1}{\rho+p(\rho)} \neq 0
$$

except for very peculiar isolated cases. Therefore, the conditions for employing the inverse function theorem apply, and thus we can write

$$
\rho=f^{-1}(a)
$$

which we often just abbreviate by writing $\rho(a)$.

The Friedmann equation can now be written as:

$$
\rho(a(t))=3\left(\frac{\dot{a}^{2}(t)}{a^{2}(t)}+\frac{k}{a^{2}(t)}\right)
$$

Equation (3.167) can be rearranged into equation (3.168) and formally solved by integration to find $t(a)$, time as a function of scale parameter $a$. First note

$$
\frac{1}{3} \rho(a(t)) a^{2}(t)-\dot{a}^{2}(t)=k .
$$

Theorem 23. Let the time now be $t_{0}$ and the size of the universe now be $a_{0}$, the standard solution of equation (3.168) in the current epoch is:

$$
t(a)=\int_{a_{0}}^{a} \frac{d \bar{a}}{\sqrt{\frac{1}{3} \rho(\bar{a}) \bar{a}^{2}-k}}+t_{0} .
$$

Proof. There are three solutions to equation (3.168): 
1.

$$
\dot{a}(t)=0 \Rightarrow \rho(a(t)) a^{2}(t) / 3=k,
$$

this means that the size of the universe does not change over time. This case is the Einstein static universe. A more complicated analysis would show that for "normal" matter the Einstein static universe is always unstable. It might be possible to find some form of "unusual" matter to make the Einstein static universe stable.

2 .

$$
t(a)=-\int^{a} \frac{d \bar{a}}{\sqrt{\frac{1}{3} \rho(\bar{a}) \bar{a}^{2}-k}}+\text { constant }
$$

in this case

$$
\frac{1}{\sqrt{\frac{1}{3} \rho(\bar{a}) \bar{a}^{2}-k}}>0 \Rightarrow \int^{a} \frac{d \bar{a}}{\sqrt{\frac{1}{3} \rho(\bar{a}) \bar{a}^{2}-k}}>0 .
$$

Time decreases as the integral increases. That is time increases as the integral decreases (as the scale factor $a$ decreases). So time increases as the universe gets smaller. This represents a contracting universe. Our universe is not currently contracting, but it is conceivable that the cosmological expansion could achieve a maximum, then turn around, and start to contract. If this happens then we would want to use this solution during the contracting phase.

3.

$$
t(a)=\int^{a} \frac{d \bar{a}}{\sqrt{\frac{1}{3} \rho(\bar{a}) \bar{a}^{2}-k}}+\text { constant }
$$

this solution is the standard solution appropriate to the current cosmological epoch.

Therefore, if we define $t_{0}$ as the time now and $a_{0}$ as the size of the universe now, the solution of equation (3.168) is:

$$
t(a)=\int_{a_{0}}^{a} \frac{d \bar{a}}{\sqrt{\frac{1}{3} \rho(\bar{a}) \bar{a}^{2}-k}}+t_{0}
$$




\section{Domains of reality}

We have shown that the current standard solution to equation (3.168) contains an integral of a square root $\sqrt{\rho(a) a^{2} / 3-k}$. However, this integral exists and is real only if the term inside the square root is positive.

There are at least 5 situations wherein the square root $\sqrt{\rho(a) a^{2} / 3-k}$ is real:

1. The square root is real only on $\left[0, a_{\max }\right]$. (This corresponds to a big bang, and then a turnaround and a possible recollapse, or a future sudden singularity.)

2. The square root is real only on $\left[a_{\star}, \infty\right]$. (This corresponds to a bounce or a past sudden singularity then followed by infinite expansion.)

3. The square root is real on the entire positve axis $[0, \infty]$. (This is the most common assumption in cosmology, it corresponds to a big bang, then followed by infinite expansion).

4. The square root is real only on a finite interval bounded away from zero, $\left[a_{\star}, a_{\max }\right]$. (This corresponds to a bounce or past sudden singularity subsequently followed by a turnaround or future sudden singularity; a possible oscillating universe...)

5. Disjoint unions of various of the above. (Not physically interesting.)

\subsubsection{Age of the universe $\tau$}

In this section, we will concentrate on the lower bound of the intervals of the different cases mentioned above, where the square root $\sqrt{\rho(a) a^{2} / 3-k}$ is real. But first, we will present a generic definition and a generic theorem on the age of the universe.

Definition 11. The age of the universe $\tau$ is the time elapsed since $a=a_{\odot}$.

Here $a_{\odot}$ can represent a big bang (in this case $a_{\odot}=0$ ) or a minimum value (for a bounce or past sudden singularity in this case $a_{\odot}=a_{\star}$ ). 
Theorem 24. The age of the universe $\tau$ is given by,

$$
\tau=\int_{a_{\odot}}^{a_{0}} \frac{d \bar{a}}{\sqrt{\frac{1}{3} \rho(\bar{a}) \bar{a}^{2}-k}} .
$$

Proof. Define $t_{\odot}=t\left(a=a_{\odot}\right)$ then

$$
t_{\odot}=\int_{a_{0}}^{a_{\odot}} \frac{d \bar{a}}{\sqrt{\frac{1}{3} \rho(\bar{a}) \bar{a}^{2}-k}}+t_{0},
$$

where $t_{0}$ is the time now. The age of the universe is therefore given by

$$
\tau=t_{0}-t_{\odot}=\int_{a_{\odot}}^{a_{0}} \frac{d \bar{a}}{\sqrt{\frac{1}{3} \rho(\bar{a}) \bar{a}^{2}-k}} .
$$

Corollary 1. Assuming the universe starts with a big bang, the age of the universe $\tau$ is the time elapsed since $a=0$, and $\tau$ is given by,

$$
\tau=\int_{0}^{a_{0}} \frac{d \bar{a}}{\sqrt{\frac{1}{3} \rho(\bar{a}) \bar{a}^{2}-k}} .
$$

Corollary 2. Assuming the universe starts with a bounce or a past sudden singularity, the age of the universe $\tau$ is the time elapsed since a minimum value $a=a_{\star}$, and $\tau$ is given by,

$$
\tau=\int_{a_{\star}}^{a_{0}} \frac{d \bar{a}}{\sqrt{\frac{1}{3} \rho(\bar{a}) \bar{a}^{2}-k}} .
$$

Theorem 25. The age of the universe $\tau$ is real and finite, that is, $\tau<\infty$ and $\tau \in \mathbb{R}$, if only if the integral

$$
\int_{a_{\odot}}^{a_{0}} \frac{d \bar{a}}{\sqrt{\frac{1}{3} \rho(\bar{a}) \bar{a}^{2}-k}}
$$

is both real and convergent.

That is, we demand both: 
1. for reality:

$$
\rho(a)>\frac{3 k}{a^{2}}
$$

2. for convergence:

there exist constants $\alpha<1, c>0$ and $a_{\epsilon}>0$ such that: $\forall a \in\left(a_{\odot}, a_{\epsilon}\right)$

$$
\rho(a) \geq \frac{3 k}{a^{2}}+c^{2} a^{2 \alpha-2}
$$

and $\forall a \in\left(a_{\epsilon}, a_{0}\right)$

$$
\rho(a) \geq \rho\left(a_{\epsilon}\right)
$$

Proof. $\quad$ 1. First, $\tau \in \mathbb{R}$ if

$$
\int_{a_{\odot}}^{a_{0}} \frac{d \bar{a}}{\sqrt{\frac{1}{3} \rho(\bar{a}) \bar{a}^{2}-k}} \in \mathbb{R}
$$

that is, if

$$
\rho(a) a^{2} / 3-k>0,
$$

which leads to the reality condition:

$$
\rho(a)>\frac{3 k}{a^{2}} .
$$

2. Second, $\tau<\infty$ if there exist constants $\alpha<1, c>0$ and $a_{\epsilon}>0$ such that: $\forall a \in\left(a_{\odot}, a_{\epsilon}\right)$

$$
\sqrt{\rho(a) a^{2} / 3-k} \geq c a^{\alpha} \geq 0,
$$

that is

$$
\rho(a) \geq \frac{3 k}{a^{2}}+c^{2} a^{2 \alpha-2} ;
$$

and, if furthermore $\forall a \in\left(a_{\epsilon}, a_{0}\right)$

$$
\sqrt{\rho(a) a^{2} / 3-k} \geq \sqrt{\rho\left(a_{\epsilon}\right) a_{\epsilon}^{2} / 3-k},
$$

that is

$$
\rho(a) \geq \rho\left(a_{\epsilon}\right) .
$$




\subsubsection{Remaining lifetime of the universe $T$}

Definition 12. The remaining lifetime of the universe $T$ is the remaining time from now, where $a=a_{0}$, until $a_{\infty}=a_{\text {end }}$.

Here $a_{\text {end }}$ can represent a big rip or eternal expansion at a finite non zero rate (in this case $a_{\text {end }}=\infty$ ), or a maximum value (for a turnaround or future sudden singularity or eternal expansion asymptotic to zero velocity in this case $a_{\text {end }}=a_{\max }$ ).

Theorem 26. The remaining lifetime of the universe $T$ is given by,

$$
T=\int_{a_{0}}^{a_{\mathrm{end}}} \frac{d \bar{a}}{\sqrt{\frac{1}{3} \rho(\bar{a}) \bar{a}^{2}-k}}
$$

Proof. Define $t\left(a_{\infty}\right)$ the time when $a_{\infty}=a_{\text {end }}$, then

$$
t\left(a_{\infty}\right)=\int_{a_{0}}^{a_{\text {end }}} \frac{d \bar{a}}{\sqrt{\frac{1}{3} \rho(\bar{a}) \bar{a}^{2}-k}}+t_{0} .
$$

Therefore, the remaining lifetime of the universe $T$ is given by

$$
T=t\left(a_{\infty}\right)-t_{0}=\int_{a_{0}}^{a_{\text {end }}} \frac{d \bar{a}}{\sqrt{\frac{1}{3} \rho(\bar{a}) \bar{a}^{2}-k}} .
$$

Corollary 3. Assuming the universe ends with a big rip, the remaining lifetime of the universe $T$ is the remaining time from now $a=a_{0}$ until $a_{\mathrm{end}}=\infty$, and $T$ is given by,

$$
T=\int_{a_{0}}^{\infty} \frac{d \bar{a}}{\sqrt{\frac{1}{3} \rho(\bar{a}) \bar{a}^{2}-k}} .
$$

Corollary 4. Assuming the universe continues to expand at a finite nonzero rate for all eternity then, the remaining lifetime of the universe $T$ diverges. Here $T$ is the remaining time from now $a=a_{0}$ until $a_{\mathrm{end}}=\infty$, and $T$ is given by,

$$
T=\int_{a_{0}}^{\infty} \frac{d \bar{a}}{\sqrt{\frac{1}{3} \rho(\bar{a}) \bar{a}^{2}-k}} .
$$


Corollary 5. Assuming the universe ends by reaching a maximum value (possible turnaround or future sudden singularity), the remaining lifetime of the universe $T$ is the remaining time from now $a=a_{0}$ until $a_{\mathrm{end}}=a_{\mathrm{max}}$, and $T$ is given by,

$$
T=\int_{a_{0}}^{a_{\max }} \frac{d \bar{a}}{\sqrt{\frac{1}{3} \rho(\bar{a}) \bar{a}^{2}-k}} .
$$

Theorem 27. The remaining lifetime of the universe $T$ is real and finite, that is, $T<\infty$ and $T \in \mathbb{R}$, if and only if the integral

$$
\int_{a_{0}}^{a_{\text {end }}} \frac{d \bar{a}}{\sqrt{\frac{1}{3} \rho(\bar{a}) \bar{a}^{2}-k}}
$$

is both real and convergent.

That is, we demand both

1. for reality:

$$
\rho(a)>\frac{3 k}{a^{2}}
$$

2. for convergence:

(a) case $a_{\mathrm{end}}=\infty$ : If there exist constants $\beta>1, c>0$ and $a_{\epsilon}>0$ such that: $\forall a \in\left(a_{\epsilon}, a_{\text {end }}\right)$

$$
\rho(a) \geq \frac{3 k}{a^{2}}+c^{2} a^{2 \beta-2}
$$

(b) case $a_{\mathrm{end}}=a_{\max }$ : If there exists constants $\alpha<1, K>0$ and $a_{\epsilon}>0$ such that: $\forall a \in\left(a_{\epsilon}, a_{\max }\right)$

$$
\rho(a) \geq \frac{3 k}{a^{2}}+\frac{3\left(a_{\max }-a\right)^{2 \alpha}}{K^{2} a^{2}}>\frac{3 k}{a_{\max }^{2}}+\frac{3\left(a_{\max }-a\right)^{2 \alpha}}{K^{2} a_{\max }^{2}} .
$$

And finally, $\forall a \in\left(a_{0}, a_{\epsilon}\right)$

$$
\rho(a) \geq \rho\left(a_{\epsilon}\right) .
$$


Proof. $\quad$ 1. First, $T \in \mathbb{R}$ if

$$
\int_{a_{0}}^{a_{\mathrm{end}}} \frac{d \bar{a}}{\sqrt{\frac{1}{3} \rho(\bar{a}) \bar{a}^{2}-k}} \in \mathbb{R}
$$

that is, if

$$
\rho(a) a^{2} / 3-k>0
$$

which leads to the reality condition:

$$
\rho(a)>\frac{3 k}{a^{2}}
$$

2. Second,

(a) case $a_{\mathrm{end}}=\infty$ : $T<\infty$ if there exist constants $\beta>1, c>0$ and $a_{\epsilon}>0$ such that: $\forall a \in\left(a_{\epsilon}, a_{\mathrm{end}}\right)$

$$
\sqrt{\rho(a) a^{2} / 3-k} \geq c a^{\beta} \geq 0
$$

that is

$$
\rho(a) \geq \frac{3 k}{a^{2}}+c^{2} a^{2 \beta-2} .
$$

(b) case $a_{\text {end }}=a_{\text {max }}: T<\infty$ if there exist constants $\alpha<1, K>0$ and $a_{\epsilon}>0$ such that: $\forall a \in\left(a_{\epsilon}, a_{\max }\right)$

$$
|f(a)| \leq \frac{K}{\left(a_{\max }-a\right)^{\alpha}}
$$

that is,

$$
\rho(a) \geq \frac{3 k}{a^{2}}+\frac{3\left(a_{\max }-a\right)^{2 \alpha}}{K^{2} a^{2}}>\frac{3 k}{a_{\max }^{2}}+\frac{3\left(a_{\max }-a\right)^{2 \alpha}}{K^{2} a_{\max }^{2}} .
$$

And, if furthermore $\forall a \in\left(a_{0}, a_{\epsilon}\right)$

$$
\sqrt{\rho(a) a^{2} / 3-k} \geq \sqrt{\rho\left(a_{\epsilon}\right) a_{\epsilon}^{2} / 3-k},
$$

that is

$$
\rho(a) \geq \rho\left(a_{\epsilon}\right) .
$$




\subsubsection{Total age of the universe $T_{\text {total }}$}

Definition 13. The total age of the universe $T_{\text {total }}$ is the time elapsed since $a=a_{\odot}$ until $a_{\infty}=a_{\mathrm{end}}$.

Lemma 7. The total age of the universe $T_{\text {total }}$ is the sum of the age of the universe $\tau$ and the remaining lifetime of the universe $T$, that is,

$$
T_{\text {total }}=\tau+T
$$

Lemma 8. The total age of the universe $T_{\text {total }}$ is real and finite if and only if:

1. the present age of the universe is real and finite $\tau \in \mathbb{R}$ and $\tau<\infty$,

2. and the remaining time of the universe is real and finite $T \in \mathbb{R}$ and $T<\infty$.

Theorem 28. The total age of the universe $T_{\text {total }}$ is given by,

$$
T_{\text {total }}=\int_{a_{\odot}}^{a_{\text {end }}} \frac{d \bar{a}}{\sqrt{\frac{1}{3} \rho(\bar{a}) \bar{a}^{2}-k}} .
$$

Proof. Theorem 28 is deduced from Theorem 24 and Theorem 26.

Theorem 29. The total age of the universe $T_{\text {total }}$ is real and finite, that is, $T_{\text {total }}<\infty$ and $T_{\text {total }} \in \mathbb{R}$, if and only if the integral

$$
\int_{a_{\odot}}^{a_{\text {end }}} \frac{d \bar{a}}{\sqrt{\frac{1}{3} \rho(\bar{a}) \bar{a}^{2}-k}}
$$

is both real and convergent.

That is, we demand both

1. for reality:

$$
\rho(a)>\frac{3 k}{a^{2}}
$$

2. for convergence: 
(a) if there exist constants $\alpha<1, c>0$ and $a_{\epsilon}>0$ such that: $\forall a \in\left(a_{\odot}, a_{\epsilon}\right)$

$$
\rho(a) \geq \frac{3 k}{a^{2}}+c^{2} a^{2 \alpha-2}
$$

(b) i. case $a_{\mathrm{end}}=\infty$ : If there exist constants $\beta>1, c>0$ and $a_{\epsilon}>0$ such that: $\forall a \in\left(a_{\epsilon}, a_{\mathrm{end}}\right)$

$$
\rho(a) \geq \frac{3 k}{a^{2}}+c^{2} a^{2 \beta-2} .
$$

ii. case $a_{\mathrm{end}}=a_{\mathrm{max}}$ : If there exists constants $\alpha<1, K>0$ and $a_{\epsilon}>0$ such that: $\forall a \in\left(a_{\epsilon}, a_{\max }\right)$

$$
\rho(a) \geq \frac{3 k}{a^{2}}+\frac{3\left(a_{\max }-a\right)^{2 \alpha}}{K^{2} a^{2}}>\frac{3 k}{a_{\max }^{2}}+\frac{3\left(a_{\max }-a\right)^{2 \alpha}}{K^{2} a_{\max }^{2}} .
$$

Proof. Theorem 29 is deduced from Theorem 25 and Theorem 27.

Now, applying the generic Theorem 29 to the five cases where the square root $\sqrt{\rho(a) a^{2} / 3-k}$ is real, we obtain the following corollaries:

Corollary 6. Assume that the square root is real only on $\left[0, a_{\max }\right]$ and $a_{\max }>a_{0}$.

If there exist constants $\alpha<1, c>0$ and $a_{\epsilon}>0$ such that: $\forall a \in\left(0, a_{\epsilon}\right)$

$$
\rho(a) \geq \frac{3 k}{a^{2}}+c^{2} a^{2 \alpha-2}>\frac{3 k}{a^{2}}
$$

and if there exists constants $\alpha<1, K>0$ and $a_{\epsilon}>0$ such that: $\forall a \in\left(a_{\epsilon}, a_{\max }\right)$

$$
\rho(a) \geq \frac{3 k}{a^{2}}+\frac{3\left(a_{\max }-a\right)^{2 \alpha}}{K^{2} a^{2}}>\frac{3 k}{a_{\max }^{2}}+\frac{3\left(a_{\max }-a\right)^{2 \alpha}}{K^{2} a_{\max }^{2}}
$$

then

$$
T_{\text {total }}<\infty \quad \text { and } \quad T_{\text {total }} \in \mathbb{R} \text {. }
$$

Corollary 6 deals with a universe that starts with a big bang $\left(a_{\odot}=0\right)$, and tends to a maximum size $\left(a_{\mathrm{end}}=a_{\max }\right)$ in a finite time. This universe could possibly have a turnaround and contracting phase until a big crunch $a=0$ or endure a bounce $a=a_{\star}$. 
Corollary 7. Assume that the square root is real only on $\left[a_{\star}, \infty\right]$.

Here $a=0$ is not possible, hence there is a bounce or a past sudden singularity.

If there exist constants $\alpha<1, c>0$ and $a_{\epsilon}>0$ such that: $\forall a \in\left(a_{\star}, a_{\epsilon}\right)$

$$
\rho(a) \geq \frac{3 k}{a^{2}}+c^{2} a^{2 \alpha-2}>\frac{3 k}{a^{2}}
$$

and if there exists constants $\beta>1, c>0$ and $a_{\epsilon}>0$ such that: $\forall a \in\left(a_{\epsilon}, \infty\right)$

$$
\rho(a) \geq \frac{3 k}{a^{2}}+c^{2} a^{2 \beta-2}>\frac{3 k}{a^{2}}
$$

then

$$
T_{\text {total }}<\infty \quad \text { and } \quad T_{\text {total }} \in \mathbb{R} .
$$

Corollary 7 deals with a universe that starts with a bounce or a past sudden singularity (minimum value $a_{\odot}=a_{\star}$ ), and ends with a big rip (infinite value $a_{\text {end }}=\infty$ ) in a finite time.

Corollary 8. Assume that the square root is real on the entire positive axis $[0, \infty]$.

If there exist constants $\alpha<1, c>0$ and $a_{\epsilon}>0$ such that: $\forall a \in\left(0, a_{\epsilon}\right)$

$$
\rho(a) \geq \frac{3 k}{a^{2}}+c^{2} a^{2 \alpha-2}>\frac{3 k}{a^{2}} ;
$$

and if there exists constants $\beta>1, c>0$ and $a_{\epsilon}>0$ such that: $\forall a \in\left(a_{\epsilon}, \infty\right)$

$$
\rho(a) \geq \frac{3 k}{a^{2}}+c^{2} a^{2 \beta-2}>\frac{3 k}{a^{2}}
$$

then

$$
T_{\text {total }}<\infty \quad \text { and } \quad T_{\text {total }} \in \mathbb{R} .
$$

Corollary 8 deals with a universe that starts with a big bang $\left(a_{\odot}=0\right)$ and ends with a big rip $\left(a_{\mathrm{end}}=\infty\right)$ in a finite time.

Corollary 9. Assume that the square root is real only on a finite interval bounded away from zero, $\left[a_{\star}, a_{\max }\right]$ and $a_{\star}<a_{0}<a_{\max }$.

If there exist constants $\alpha<1, c>0$ and $a_{\epsilon}>0$ such that: $\forall a \in\left(a_{\star}, a_{\epsilon}\right)$

$$
\rho(a) \geq \frac{3 k}{a^{2}}+c^{2} a^{2 \alpha-2}>\frac{3 k}{a^{2}}
$$


and if there exists constants $\alpha<1, K>0$ and $a_{\epsilon}>0$ such that: $\forall a \in\left(a_{\epsilon}, a_{\max }\right)$

$$
\rho(a) \geq \frac{3 k}{a^{2}}+\frac{3\left(a_{\max }-a\right)^{2 \alpha}}{K^{2} a^{2}}>\frac{3 k}{a_{\max }^{2}}+\frac{3\left(a_{\max }-a\right)^{2 \alpha}}{K^{2} a_{\max }^{2}}
$$

then

$$
T_{\text {total }}<\infty \quad \text { and } \quad T_{\text {total }} \in \mathbb{R} \text {. }
$$

Corollary 9 deals with a universe that starts with a bounce or a past sudden singularity (minimum value $a_{\odot}=a_{\star}$ ) and ends with a turnaround or a future sudden singularity (maximum value $a_{\text {end }}=a_{\max }$ ). This universe could possibly have a turnaround and contracting phase until a big crunch $a=0$ or endure a bounce $a=a_{\star}$.

Corollary 10. Disjoint unions of various of the above.

This corollary corresponds to some of the cases described in the above corollaries.

This case is not physically interesting since it corresponds to several unrelated universes that are totally independent of each other.

\subsubsection{Summary}

In this section, we have presented definitions and theorems regarding the conditions that should be placed on the the density as a function of the scale factor in order for the integral

$$
T_{\text {total }}=\int_{a \odot}^{a_{\text {end }}} \frac{d \bar{a}}{\sqrt{\frac{1}{3} \rho(\bar{a}) \bar{a}^{2}-k}}
$$

to converge. We obtain model independent results that place constraints on $\rho(a)$ under a minimum of technical assumptions. Whether or not the total age of the universe is a real and finite number depends on whether these conditions on the density $\rho(a)$ hold or not. 


\subsection{Results and discussion}

In this chapter we have explored three issues concerning singularities in a FRW universe using only classical general relativity and an absolute minimum of technical input assumptions:

- First we have developed an extensive catalogue of the various "cosmological milestones" found in the literature in terms of a generalized power series expansion of the FRW scale factor.

If in the vicinity of any cosmological milestone, the input scale factor $a(t)$ is a generalized power series, then all physical observables ( $H, q$, the Riemann tensor, etc.) will likewise be generalized power series, with related indicial exponents that can be calculated from the indicial exponents of the scale factor. Whether or not the particular physical observable then diverges at the cosmological milestone is "simply" a matter of calculating its dominant indicial exponent in terms of those occuring in the scale factor.

Indeed, for some unspecified generic cosmological milestone, that is defined in terms of the behaviour of the scale factor $a(t)$, and which occurs at some finite time $t_{\odot}$, we can write in the vicinity of the milestone:

$$
a(t)=c_{0}\left|t-t_{\odot}\right|^{\eta_{0}}+c_{1}\left|t-t_{\odot}\right|^{\eta_{1}}+c_{2}\left|t-t_{\odot}\right|^{\eta_{2}}+c_{3}\left|t-t_{\odot}\right|^{\eta_{3}}+\ldots
$$

The $\eta_{i}$ are generically real and ordered such that $\eta_{0}<\eta_{1}<\eta_{2}<\eta_{3} \ldots$ Also $c_{0}>0$ though there are no a priori constraints on the signs of the other $c_{i}$, except by definition $c_{i} \neq 0$.

This generalized power series expansion is sufficiently general to accommodate all commonly occurring models considered in the literature. Specifically,

- $\eta_{0}>0$ holds for the class of cosmological milestones such as big bangs or big crunches;

- $\eta_{0}<0$ holds for the class of cosmological milestones such as big rips;

- $\eta_{0}=0$ holds for the class of cosmological milestones such as sudden singularities or extremality events (bounces, turnarounds, inflexions); 
Note that for extremality events the scale factor can be modeled using ordinary Taylor series with $\eta_{i} \in Z^{+}$.

- Second, with the notion of a generalized power series in hand, it is possible at a purely kinematic level to address the question of when a "cosmological milestone" corresponds to a curvature singularity, and what type of singularity is implied.

In section 3.2.2, we have shown that the Hubble parameter $H(t)$ blows up in the vicinity of cosmological milestones such as big bangs/crunches and big rips (Theorem 3), however it is finite for a specific class of sudden singularities/extremality events (Theorem 4), specifically,

$$
\lim _{t \rightarrow t_{\odot}} H=\left\{\begin{array}{lll}
+\infty & \eta_{0}>0 ; & \\
0 & \eta_{0}=0 ; & \eta_{1}>1 ; \\
c_{1} / c_{0} & \eta_{0}=0 ; & \eta_{1}=1 ; \\
\operatorname{sign}\left(c_{1}\right) \infty & \eta_{0}=0 ; & \eta_{1} \in(0,1) ; \\
-\infty & \eta_{0}<0 . &
\end{array}\right.
$$

where $t_{\odot}$ is the the time of the event.

In the same section, we have also demonstrated that the deceleration parameter $q(t)$ is always finite for bangs, crunches and rips $\left(\eta_{0} \neq 0\right)$ but it is infinite only for a certain subset of the sudden singularities

$$
\lim _{t \rightarrow t_{\odot}} q=\left\{\begin{array}{llll}
\left(1-\eta_{0}\right) / \eta_{0} & \eta_{0} \neq 0 ; & \\
\operatorname{sign}\left(c_{1}\left[1-\eta_{1}\right]\right) \infty & \eta_{0}=0 ; & \eta_{1} \neq 1 ; & \\
0 & \eta_{0}=0 ; & \eta_{1}=1 ; & \eta_{2}>2 ; \\
-2 c_{2} c_{0} / c_{1}^{2} & \eta_{0}=0 ; & \eta_{1}=1 ; & \eta_{2}=2 ; \\
-\operatorname{sign}\left(c_{2}\right) \infty & \eta_{0}=0 ; & \eta_{1}=1 ; & \eta_{2} \in(1,2),
\end{array}\right.
$$

where $t_{\odot}$ is the the time of the event.

We have considered the Riemann tensor and analysed its behaviour, in this way it is possible to classify all "cosmlogical milestones" as to whether they are polynomial curvature singularities or not: there are only a very few cases of cosmological milestones that are not polynomial curvature singularities. In Theorem 
9, we mention that those cases correspond to sudden singularities/extremality events $\left(\eta_{0}=0\right)$ and asymptotically Milne big bangs/crunches. In detail, the only cases where cosmological milestones are not polynomial curvature singularities are:

$$
\begin{array}{lll}
-\eta_{0}=0 & \eta_{1} \geq 2 \text { or } & \\
-\eta_{0}=0 & \eta_{1}=1 & \eta_{2} \geq 2 \text { or } \\
-\eta_{0}=1 & \eta_{1} \geq 3 & \text { and } \quad k=-1, c_{0}=1 .
\end{array}
$$

However, when looking at time derivatives of the curvature tensor, most of these limited cases disappear: the only two situations in which a cosmological milestone is not a derivative curvature singularity are if:

$-\eta_{0}=0, \eta_{i} \in Z^{+}$; corresponding to an extremality event;

- $\eta_{0}=1, k=-1, c_{0}=1, \eta_{i} \in Z^{+}$, and $\eta_{1} \geq 3$; corresponding to a FRW geometry that smoothly asymptotes near the cosmological milestone to the Riemann-flat Milne universe.

- Third, this definition of cosmological milestones in terms of generalized power series enables us to perform a complete model-independent check on the validity or otherwise of the classical energy conditions.

In particular we provide a complete catalogue of those bangs/crunches, sudden singularities and extremality events for which the NEC, the WEC, the SEC and the DEC are satisfied.

Depending on one's attitude towards the energy conditions [3], one could use this catalogue as a guide towards deciding on potentially interesting scenarios to investigate. In particular,

- The NEC, the WEC and the SEC hold independent of the sign of space curvature $k$ all the way down to the singularity, for big bangs/crunches for which $0<\eta_{0}<1$. That is, in the vicinity of the singularity the dominant term in the scale factor $a(t)$ is bounded from both above and below by

$$
c_{0}\left(t-t_{*}\right)<a_{\text {dominant }}(t) \leq c_{0} .
$$


- "Big rips" $\left(\eta_{0}<0\right)$ will violate the NEC in the vicinity of the milestone and therefore, they will also violate every other energy conditions.

- The SEC will be violated for all "violent" bangs and crunches with $\eta_{0}>1$, though the NEC and the WEC will still be satisfied in this case.

- The DEC holds independent of the sign of space curvature $k$ all the way down to the singularity, for big bangs/crunches for which $1 / 3<\eta_{0}<1$. That is, in the vicinity of the singularity the dominant term in the scale factor $a(t)$ is bounded from both above and below by

$$
c_{0}\left(t-t_{*}\right)<a_{\text {dominant }}(t) \leq c_{0}\left(t-t_{*}\right)^{1 / 3} .
$$

- The DEC is also satisfied for a limited range of sudden singularities $\left(\eta_{0}=0\right)$. Sudden singularities of this type are sufficiently "gentle" that at least for some of them even the DEC can be satisfied all the way to the singularity: a conclusion completely in agreement with Barrow and Tsagas [8].

Writing the scale factor as a generalized power series expansion has allowed us to derive important and very general results on the various cosmological milestones both on a kinematical and dynamical levels. These theorems have been developed with an absolute minimum of technical imput. Simply by calculating the dominant indicial exponent of the scale factor we have been able to determine whether or not some particular physical observable (expressed as power series) diverges at the cosmological milestone. 


\section{Chapter 4}

\section{Gravastars}

This chapter introduces the reader to the idea of gravastars, how they are configured, as well as some of their properties. We present results deduced from these properties but we do so from an agnostic point of view as to the existence or non-existence of gravastars.

The study and understanding of the features of gravastars are important to comprehend what observational data regarding astrophysical black holes are telling us.

\subsection{Definitions}

\subsubsection{GRAvitational VAcuum STARS}

The concept of black hole ${ }^{1}$ is generally accepted in the general relativity community, but one sometimes encounter some scepticism concerning the reality of the mathematical solution and wariness regarding the interpretation of observational data [1]. The Schwarzschild solution presents a central singularity at $r=0$ and an event horizon at the Schwarzschild radius ${ }^{2} R_{\text {Schwarzschild }}=2 M$.

The GRAvitational VAcuum STAR (gravastar) model is a proposal by Emil Mottola and Pawel Mazur $[57,58,59]$ to replace black holes. Instead of having a star

\footnotetext{
${ }^{1}$ See section 2.5.1 for more details.

${ }^{2}$ See section 2.5.2 for more details.
} 
contracting and collapsing until matter arrives at a singularity at the centre, the gravastar model suggests that a gravitationally collapsing star would force spacetime itself to undergo a phase transition that would prevent further collapse. Thus the star would be transformed into a spherical "quantum vacuum" surrounded by a form of super-dense matter.

It has been speculated that violent creation of a gravastar might be an alternate explanation for gamma ray bursts. Gravastars could also be a solution for the black hole information paradox ${ }^{3}$. The Gravastar is theorized to have very low amounts of entropy, on the contrary, a black hole apparently has a billion times more entropy than the star it formed from. A problem with the theory regarding the creation of a gravastar is whether or not a star would be capable of shedding enough entropy upon implosion. Externally, a gravastar appears similar to a black hole: it is visible only by the high-energy emissions it creates while consuming matter. Astronomers observe the sky for X-rays emitted by infalling matter to detect black holes, and a gravastar would produce a similar signature.

\subsubsection{Common models}

In the Mazur-Mottola model, the concept of Bose-Einstein condensation ${ }^{4}$ in gravitational systems is extended to compact object with an interior de Sitter space (with an equation of state $\rho=-p>0)$, and an outer region of the gravastar consisting of a (relatively thin) finite-thickness shell of stiff matter $(p=\rho)$. The exterior of the gravastar is surrounded by a Schwarzschild vacuum geometry $(p=\rho=0)$.

In addition to these three layers, the model requires two infinitesimally-thin shells

\footnotetext{
${ }^{3}$ The black hole information paradox results from the combination of quantum mechanics and general relativity. In 1975, Stephen Hawking showed that black holes should slowly radiate away energy, which poses a problem. From the no hair theorem one would expect the Hawking radiation to be completely independent of the material entering the black hole. However, if the material entering the black hole were a pure quantum state, the transformation of that state into the mixed state of Hawking radiation would destroy information about the original quantum state. This violates the rules of standard quantum mechanics and presents a seeming physical paradox.

${ }^{4}$ In Bose-Einstein condensate, all matter (protons, neutrons, electrons, etc...) goes into what is called a quantum state creating a "super-atom".
} 
with surface densities $\sigma_{ \pm}$, and surface tensions $\vartheta_{ \pm}$. These compensate the discontinuities in the pressure profile and stabilize this 5-layer construction, introducing delta-function anisotropic pressures [57, 58, 59].

\subsubsection{Problem}

Is it possible to replace the thin shell (from the Mazur-Mottola model) completely with a continuous layer of finite thickness? For physical reasons, we find it useful to minimize the use of thin shells as they really are a mathematical abstraction.

Is the pressure anisotropy (implicit in the Mazur-Mottola infinitesimally thin shell) a necessity for any gravastar-like objects? Is it really possible to build a gravastar-like objects using only perfect fluid with a continuous layer of finite thickness? Could a horizon or naked singularity form and why?

Assuming pressure is continuous and differentiable, we will first analyze the resulting static geometry with the resulting isotropy TOV equation (with isotropic pressure) and then the resulting anisotropy TOV equation (anisotropic pressure).

\subsection{Properties}

\subsubsection{A static spherically symmetric geometry}

To describe the geometry of the gravastar, we use spherical coordinates $(t, r, \theta, \phi)$ and we assume that the geometry is static, and spherically symmetric.

- A static solution means that there exists a hypersurface orthogonal Killing vector that is timelike near spatial infinity. This implies that the spacetime metric $d s^{2}$ can be chosen to be invariant under a time reversal about any origin of time, e.g. all cross terms $d x^{t} d x^{i} \forall i \neq t$ vanish in the chosen coordinate system.

- A spherically symmetric solution means that there exists a privileged point, e.g. the origin, such that the system is invariant under spatial rotations about the origin. 
The most general static spherically symmetric line element in four dimensions can be written in the canonical form [79]:

$$
d s^{2}=-f(r) d t^{2}+h(r) d r^{2}+r^{2} d \Omega,
$$

where $d \Omega^{2}=d \theta^{2}+\sin ^{2} \theta d \phi^{2}$.

For any spherically symmetric stress-energy tensor, we have:

$$
T_{a b}=\rho u_{a} u_{b}+p_{r} n_{a} n_{b}+p_{t}\left(g_{a b}+u_{a} u_{b}-n_{a} n_{b}\right),
$$

where $p_{r}$ is the radial pressure, $p_{t}$ the tangential pressure, $u^{a}$ the fluid 4 -velocity pointing in the same direction as the static Killing vector field $\xi^{a}$ (in order to be compatible with the static symmetry spacetime) and $n_{a}$ is a unit radius vector. This property of $u^{a}$ leads to a relation between the 4-velocity and the function $f$ appearing in the general metric:

$$
u^{a}=-f^{\frac{1}{2}}(d t)^{a}
$$

Now, introducing the Einstein's equations developed in section 2.3, we obtain relations between elements of the stress energy tensor and the functions $f$ and $h$ from the metric. Specifically, the equation in the $t t$ components, using "geometrized units" 5 and with orthonormal components, involve:

$$
G_{\hat{t} \hat{t}}=8 \pi T_{\hat{t} \hat{t}}=8 \pi \rho
$$

Equation (4.4) can be rewritten involving the function $h$ :

$$
\frac{1}{r^{2}} \frac{d}{r}\left(r\left(1-h^{-1}\right)\right)=8 \pi \rho .
$$

Hence, the solution for $h$ can be calculated:

$$
h(r)=\left(1-\frac{2 m(r)}{r}\right)^{-1},
$$

where ${ }^{6}$

$$
m(r)=4 \pi \int_{0}^{r} \rho(\tilde{r}) \tilde{r}^{2} d \tilde{r} .
$$

\footnotetext{
${ }^{5}$ Geometrized units mean that $G$ and $c$ are set equal to one, see section 2.3 for more details.

${ }^{6}$ Note that in order to avoid a "conical singularity" in the metric at $r=0$, (in other words if we require the geometry to be regular at $r=0$ ), then the integration constant $m_{0}$ in the equation $m(r)=4 \pi \int_{0}^{r} \rho(\tilde{r}) \tilde{r}^{2} d \tilde{r}+m_{0}$ must be set equal to 0.
} 
Therefore, the $t t$-field equation yields equation (4.7). The term $m(r)$ represents the total mass-energy inside the radius $r$ integrated from the centre $r=0$ until arbitrary $r$. Note that if $r=0$ cannot be defined (e.g., in the case of wormhole throats), the integration would be from an arbitrary constant $r_{0}$ to $r$. Also note that this equation of the mass is precisely the equation you would expect for a spherically symmetric mass in an Euclidean space.

The $r r$-field equation from the relation (2.15) of Einstein field equations, in geometrized units and with orthonormal components, gives:

$$
G_{\hat{r} \hat{r}}=8 \pi T_{\hat{r} \hat{r}}=8 \pi p_{r} .
$$

Equation (4.8) involves the $g_{t t}=-f(r)$ term of the metric, but it is more physically convenient to express $f(r)$ as a function of the quantity $g(r)$ defined below.

Definition 14. Let $g(r)$ be related to the $g_{t t}$ component of the metric as follows:

$$
\begin{aligned}
g(r) & =\frac{1}{2} \frac{d}{d r} \ln \left(-g_{t t}\right) \\
& =a^{r} g_{r r}
\end{aligned}
$$

where $a^{r}$ is the radial acceleration of an observer "at rest", which means that the 4 velocity vector is parallel to the Killing field ${ }^{7}$.

Now, after rearranging this definition, we can write the $g_{t t}$ component of the metric in terms of $g(r)$ :

$$
g_{t t}=-\exp \left(2 \int_{r}^{\infty} g(\tilde{r}) d \tilde{r}\right) .
$$

Now, after further calculations, equation (4.8), that is the $G_{r r}$ equation implies that

$$
g(r)=\frac{m(r)+4 \pi p_{r}(r) r^{3}}{r^{2}\left(1-\frac{2 m(r)}{r}\right)} .
$$

The physical meaning of $g(r)$ is that this function represents the locally measured gravitational acceleration, which is pointing inwards for positive $g(r)$. Note that equation (4.7) also means that

$$
\frac{d m}{d r}=4 \pi \rho r^{2} .
$$

\footnotetext{
${ }^{7}$ In a Killing vector field, $(r, \theta, \phi)$ is constant.
} 
Definition 15. We define the average density $\bar{\rho}=\frac{m(r)}{\frac{4 \pi}{3} r^{3}}$, so that the gravitational acceleration $g(r)$ can be written:

$$
g(r)=\frac{4 \pi r}{3} \frac{\bar{\rho}+3 p_{r}(r)}{1-\frac{2 m(r)}{r}}
$$

Finally, adopting $(t, r, \theta, \phi)$ coordinates we write any static spherically symmetric geometry, and in particular for gravastars, in the form ${ }^{8}$

$$
d s^{2}=-\exp \left(2 \int_{r}^{\infty} g(\tilde{r}) d \tilde{r}\right) d t^{2}+\frac{d r^{2}}{\left(1-\frac{2 m(r)}{r}\right)}+r^{2} d \Omega^{2} .
$$

Of course, the metric of the exterior of the gravastar is obviously the Schwarzschild exterior solution $(2.31)^{9}$.

\subsubsection{A TOV equation}

The remaining field equation $G_{\theta \theta}$ from the Einstein field equations (2.3), gives an expression for the radial pressure $p_{r}$, however, the rather messy algebra can be circumvented by the use of the Bianchi identity (2.9) to replace it with the covariant conservation equation of the stress-energy tensor:

$$
\nabla_{b} T^{a b}=0
$$

The stress-energy tensor is given in equation (4.2), after differentiating, this yields to

$$
\frac{d p_{r}}{d r}=-\left(\rho+p_{r}\right) g+\frac{2\left(p_{t}-p_{r}\right)}{r} .
$$

Equation (4.17) is known as the anisotropic Tolman-Oppenheimer-Volkoff (TOV) equation. In the case of isotropic pressure $\left(p=p_{r}=p_{t}\right)$ this leads to the more familiar isotropic TOV equation:

$$
\frac{d p}{d r}=-(\rho+p) g
$$

\footnotetext{
${ }^{8}$ This spacetime geometry is also referred to as an "interior solution".

${ }^{9}$ Remember that the unique vacuum solution to the Einstein's equations is the Schwarzschild exterior solution by Birkhoff stated in Theorem 1.
} 
which can be rewritten, using Definition 15 and the expression of the average density $\bar{\rho}$, as

$$
\frac{d p}{d r}=-\frac{(\rho+p)\left(m+4 \pi p r^{3}\right)}{r^{2}[1-2 m(r) / r]}=-\frac{4 \pi r}{3} \frac{(\rho+p)(\bar{\rho}+3 p)}{1-2 m(r) / r} .
$$

Definition 16. Let's define the dimensionless anisotropy parameter $\Delta$ for anisotropic pressures by

$$
\Delta=\frac{p_{t}-p_{r}}{\rho} .
$$

Therefore, in terms of this dimensionless parameter, the anisotropic TOV equation becomes:

$$
\frac{d p_{r}}{d r}=-\frac{4 \pi r}{3} \frac{\left(\rho+p_{r}\right)\left(\bar{\rho}+3 p_{r}\right)}{1-2 m(r) / r}+\frac{2 \rho \Delta}{r} .
$$

The TOV equation shows that pressure contributes as a source of gravity, and one can notice that it follows that the pressure is higher than in Newtonian stars of the same density profile. It also means that for a given $\rho(r)$, the central pressure $p_{c}$ required for equilibrium is always higher in general relativity than in Newtonian theory. Therefore, it is harder to maintain equilibrium in general relativity.

\section{How to solve the TOV equation?}

For full determination of the variables $p_{r}, p_{t}, \rho, m$, one needs the TOV equation (4.21) but also

- equation (4.13) which gives the derivative of the mass $d m / d r$ as a function of the density $\rho$,

- an equation of state for the radial pressure of the stellar material

$$
\rho=\rho\left(p_{r}\right)
$$

- an equation of state for the tangential pressure of the stellar material

$$
\rho=\rho\left(p_{t}\right)
$$

- boundary conditions: 
- spacetime must be everywhere locally Lorentzian and in particular at the centre of the star, it is therefore necessary that the mass vanishes at the centre $^{10}$ :

$$
m=0 \quad \text { at } \quad r=0
$$

- at the star's surface the interior spacetime geometry (4.2.1) must join smoothly to the exterior Schwarzschild geometry (2.31), therefore

$$
m=M \quad \text { at } \quad r=R_{\text {surface }},
$$

and by definition, the radial pressure $p_{r}$ is zero at the surface of the star.

The method to produce a model is straightforward providing that:

- the equations of state mentioned before are specified,

- central density $\rho_{c}$ or central pressure $p_{c}{ }^{11}$ for the star are given.

One can integrate the coupled hydrostatic equilibrium equation (4.21) and the mass equation (4.13) outward from the centre, beginning with the initial conditions $m=0$ and $p_{r}=p_{c}$ at the center. The integration terminates when the pressure falls to zero ${ }^{12}$ at the surface of the star. The value of the mass at this surface radius represents the total mass-energy $M$ that appear in the Schwarzschild exterior solution (2.31).

\section{Qualitative differences introduced by $\Delta$.}

Let's have a qualitative look at the difference that anisotropy introduces in the TOV equation with dimensionless anisotropy parameter $\Delta$. Remember that if we assume the WEC ${ }^{13}$ holds, then $\rho \geqslant 0$ everywhere in the star.

\footnotetext{
${ }^{10}$ This condition (locally Lorentz) is actually responsible for leading exactly to equation (4.7), that is $m(r)=\int_{0}^{r} 4 \pi \tilde{r}^{2} \rho d \tilde{r}$.

${ }^{11}$ Spherical symmetry requires that $p_{r}=p_{t}=p_{c}$ at the centre.

${ }^{12}$ Note that for gravastars, theoretically, the pressure starts off negative, passes through a first zero, increases to a maximum pressure before falling down to zero at the surface.

${ }^{13}$ See section 2.6.1.
} 
1. case $\Delta>0$ : (e.g. $\left.p_{r}<p_{t}\right)$

Typically, this condition holds for rigid solids, in telluric planets like the earth for example. If the radial pressure is negative, and equivalently pushing inwards, the tangential pressure, created by the rigidity of the surface, can be responsible for preventing the object from collapsing. In other words, the tangential pressure is doing more to stop gravitational collapse than the radial pressure.

2. case $\Delta<0$, and $0<p_{t}<p_{r}$ :

This particular case occurs in fluids with weak surface tension and where gravity plays an important role to hold the fluid together. More importantly, the radial pressure must be greater at the centre than it would be in a completely isotropic object as the pressure profile is steeper.

3. special case: $p_{t}<0$ :

Objects satisfying this condition are fluids with strong surface tension, such as raindrops for example. The radial pressure must also be greater at the centre than it would be in a completely isotropic object as the pressure profile is steeper. However, in this case, the radial pressure does not necessarily go to zero smoothly at the surface.

\subsubsection{Specific key features}

In this section, we present the class of spacetime geometries that we are particularly interested in for the gravastar model.

In the same spirit of Mazur and Mottola [57, 58, 59], and Laughlin et al [20, 21], we assume that the weak energy condition holds through out the configuration (i.e. the density is positive), but we permit the pressure to become negative in the gravastar interior. The density does not need to be continuous ${ }^{14}$ but to avoid infinitesimally thin shells (purpose of this model), one must demand that the radial pressure $p_{r}$ is

\footnotetext{
${ }^{14}$ Typically, the density is not continuous at the surface of the gravastar.
} 
continuous. The radial pressure is drawn qualitatively in Figure 4.1.

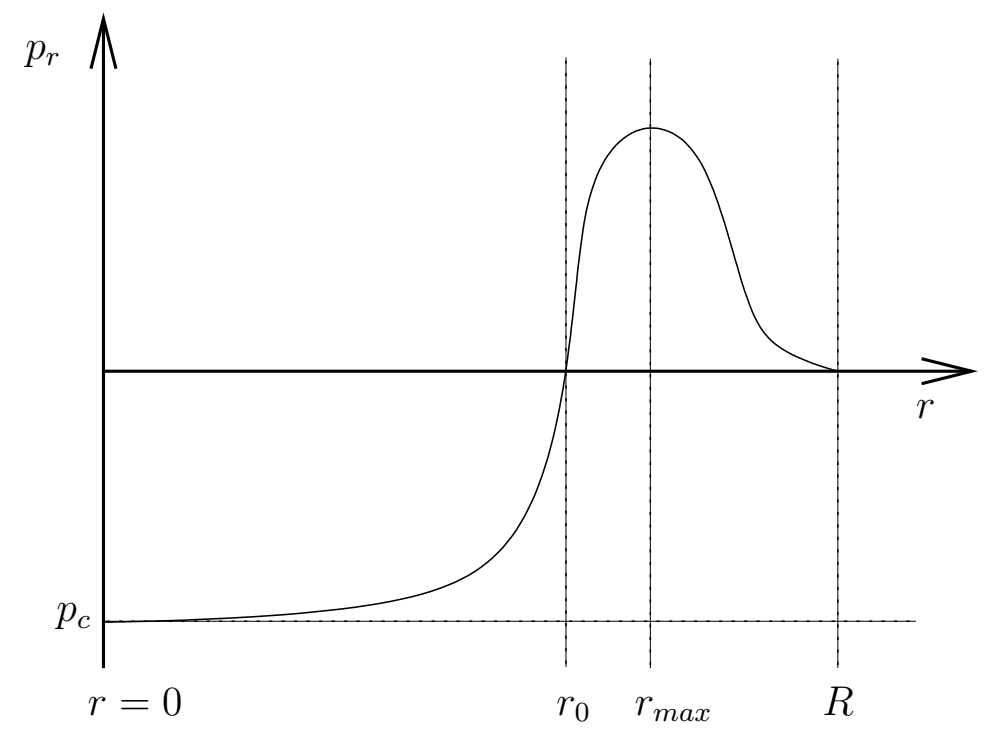

Figure 4.1: Qualitative sketch of radial pressure as a function of $r$ for a gravastar .

To "smooth out" the infinitesimally thin shells of the Mazur and Mottola gravastar model, we consider static spherically symmetric geometries such that:

- Inside the gravastar, $r<R$, the density is everywhere positive and finite.

- The central pressure is negative, $p_{c}<0$, and in fact $p_{c}=-\rho_{c}$. (We do not demand $\rho=-p_{r}=-p_{t}$ except at the centre ${ }^{15}$.)

Positive energy density but negative pressure, and origin free of any mass singularity, are the characteristic features of de Sitter space. The idea of replacing the Schwarzschild singularity with de Sitter vacuum goes back at least to 1965 . Gliner [37] interpreted $p=-\rho$ as corresponding to a "vacuum" and suggested that it could be a final state in a gravitational collapse.

\footnotetext{
${ }^{15}$ Remember that because of the spherical symmetry $p_{r}=p_{t}$ at the centre.
} 
Indeed for the the specific metric

$$
d s^{2}=-c^{2} \gamma(r) d t^{2}+\gamma^{-1}(r) d r^{2}+r^{2} d \Omega^{2},
$$

and the choice of cosmological-constant-like matter leads to

$$
R_{a b}-\frac{1}{2} g_{a b} R=\left\{\begin{array}{cc}
\frac{3}{r_{s}^{2}} g_{a b} & r<r_{s} ; \\
0 & r>r_{s}
\end{array}\right.
$$

where $r_{s}=\frac{2 G M}{c^{2}}$, with $M$ the total mass of the object, and with

$$
\gamma(r)=\left\{\begin{array}{cc}
1-\left(\frac{r}{r_{s}}\right)^{2} & r<r_{s} ; \\
1-\frac{r_{s}}{r} & r>r_{s} ;
\end{array}\right.
$$

Note that the interior de Sitter region may be also interpreted as a cosmological spacetime, with the horizon of the expanding universe replaced by a quantum phase interface. Also note the unpleasantness at $r=r_{s}$ : Laughlin et al call this a "phase transition"; Mazur-Mottola seek to smooth out the unpleasant region. Hence, the characteristics of the gravastar at the centre, are those of a de-Sitter spacetime geometry.

- The spacetime is assumed to not possess an event horizon.

This implies that $\forall r$ we have $2 m(r)<r$.

These three features, positive density, negative central pressure, and the absence of horizons, are the three most important features characterizing a gravastar.

Other important features are:

- To keep the centre of the spacetime regular, we enforce both $p_{r}^{\prime}(0)=0$ and $p_{c}=p_{r}(0)=p_{t}(0)$.

- There should be a pressure maximum in the general vicinity of the Schwarzschild $\operatorname{radius}^{16}, r_{\max } \approx R_{\text {Schwarzschild }}$, satisfying $p_{r}\left(r_{\max }\right)>0$, and $p_{r}^{\prime}\left(r_{\max }\right)=0$.

(This permits the physics in the region $r \gg r_{\max }$ to be more or less standard.)

\footnotetext{
${ }^{16}$ See section 2.5 .2
} 
- There should be exactly two radii where the radial pressure vanishes:

- The first pressure zero $p_{r}\left(r_{0}\right)=0$, where $p_{r}^{\prime}\left(r_{0}\right)>0$, and

- the second pressure zero $p_{r}(R)=0$, where $p_{r}^{\prime}(R) \leq 0$.

The point $R$, (which by construction must satisfy $R>r_{\max }>r_{0}$ ), is called the surface of the gravastar.

- The pressure profile $p_{r}(r)$ should be continuous.

(In contrast, it is sometimes useful to allow $p_{t}(r)$ to be discontinuous.)

- The strong energy condition [SEC; $\rho+p_{r}+2 p_{t} \geq 0$ ] is definitely violated, at least near the centre of the gravastar.

At the centre of the star, $p_{c}=p_{r}=p_{t}=-\rho$ with $\rho>0$, therefore

$$
\rho+p_{r}+2 p_{t}=\rho-\rho-2 \rho=-2 \rho<0 .
$$

- We choose to enforce the null energy condition [NEC; $\left.\rho+p_{i} \geq 0\right]$ throughout the gravastar. In view of our first comment that density is everywhere positive, this implies that we are enforcing the weak energy condition [WEC; $\rho+p_{i} \geq 0$ and $\rho \geq 0]$.

- We impose no restriction regarding the dominant energy condition [DEC; $\rho \geq 0$ and $\left.\left|p_{i}\right| \leq \rho\right]$, and in fact we shall see that the DEC must fail in parts of the gravastar that are sufficiently "close" to forming a horizon.

\section{Why does the radial pressure goes through two zeros?}

It is clear on Figure 4.1 that our model describes a radial pressure that starts negative at the centre, then increases till $p_{r}=0$, becomes positive, and starts decreasing after a maximum $p_{r}=p_{\max }$ at $r=r_{\max }$ eventually reaching a second zero at the surface.

By definition, when the pressure is zero it corresponds to the surface of the star so we could have smoothly joined the vacuum interior to an exterior Schwarzschild solution (2.31). The reason for not doing so is a purely pragmatic one based on the fate of infalling positive-pressure matter. If we start without a positive-pressure region 
of type $\left(r_{0}, R\right)$, then any infalling positive-pressure matter that accumulates above $r_{0}$ will automatically generate a positive-pressure region of type $\left(r_{0}, R\right)$. The only way to avoid a positive-pressure region of type $\left(r_{0}, R\right)$ is if the negative-pressure matter in the $\left(0, r_{0}\right)$ region immediately catalyzes any infalling positive-pressure matter into negative-pressure matter. In this entire chapter, we will keep the negative-pressure matter deep in the core, discretely hidden behind a layer of positive-pressure matter.

\section{How does our model relate to others?}

We have highlighted specific features that a perfect fluid sphere gravastar, with continuous pressure, should have. But how does our gravastar model relate to other features in previous models?

- In the Mazur-Mottola model, the infinitesimally thin shell interface is the limiting case where $r_{\max } \rightarrow r_{0}$, while $\rho=-p_{r}=-p_{t}$ is strictly enforced for $r<r_{0}$. Also, an additional thin shell is placed at the surface so that the radial pressure is positive on the left limit of the surface radius $p_{r}\left(R^{-}\right)>0$.

- A simplified variant of the Mazur-Mottola 3-region model is also considered in [77] but the surface radius tends to $r_{\max }$, e.g. $R \rightarrow r_{\max }$. So in this model $r_{0}=r_{\max }=R$ and there is a single thin shell at $r_{\max }$ with de Sitter geometry inside and Schwarzschild geometry outside.

- In the Laughlin et al model, $r_{0} \rightarrow R_{\text {Schwarzschild }}$ from below, while $R \rightarrow R_{\text {Schwarzschild }}$

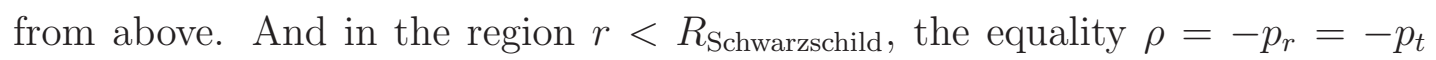
remains all the way through. There is implicitly a singular infinitesimally thin shell located exactly at $R_{\text {Schwarzschild }}$ with infinite surface tension.

- The Gliner [38] and Dymnikova [24, 25, 26, 27, 28] proposals all satisfy the constraint $\rho=-p_{r}$ everywhere throughout the configuration. We assume $\rho=$ $-p_{r}$ only at the centre, this equation does not need to hold everywhere. 


\subsection{The problem of the negative-pressure perfect fluid sphere gravastar.}

In this section, we will only consider perfect fluids, and therefore the pressure is now isotropic $p=p_{r}=p_{t}$, which means that the dimensionless anisotropy parameter is null throughout this whole section $\Delta=0$. The TOV equation is described by equation (4.19) in this section.

\subsubsection{Four inconsistencies}

In this section, we will have a look at four cases where some inconsistency within the isotropic TOV equation arise for perfect fluid sphere gravastars.

\section{First inconsistency}

Theorem 30. The isotropic TOV equation cannot hold at the point $r_{0}$ where the radial pressure is zero $p\left(r_{0}\right)=0$.

Proof. By assumption, the LHS of the TOV isotropic equation, $d p / d r$, is positive. As shown on Figure (4.1), the pressure is increasing in the region $r<r_{0}$. However, we can calculate the exact value of the pressure gradient at $r=r_{0}$ where $p=0$ :

$$
\left.\frac{d p}{d r}\right|_{r=r_{0}}=-\frac{4 \pi r_{0}}{3} \frac{\rho \bar{\rho}}{1-2 m / r_{0}} .
$$

The density is positive (as we assume the WEC) and therefore the average density $\bar{\rho}$

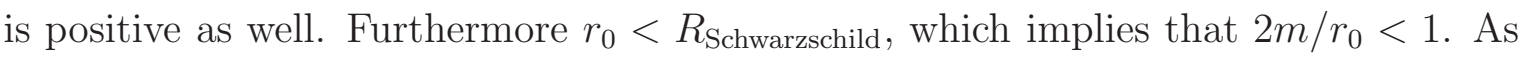
a consequence, it follows that the RHS of equation (4.30) is negative.

Therefore, there is an inconsistency, the LHS cannot be positive while the RHS is negative. Thus, the isotropic TOV cannot hold at the point $r_{0}$.

\section{Second inconsistency}

Theorem 31. The isotropic TOV equation cannot hold at the point $r_{\max }$ where the radial pressure is positive and reaches a maximum $p\left(r_{\max }\right)>0$. 
Proof. Now let's consider the point of maximum radial pressure $r_{\max }$. We can calculate the value of the TOV equation at this point:

$$
\left.\frac{\mathrm{d} p}{\mathrm{~d} r}\right|_{r=r_{\max }}=-\frac{4 \pi r_{\max }}{3} \frac{(\rho+p)(\bar{\rho}+3 p)}{1-2 m / r_{\max }} .
$$

At $r_{\max }$ the pressure is a maximum and positive for the class of models we consider, therefore $p\left(r_{\max }\right)>0$ and its derivative is zero $\mathrm{d} p /\left.\mathrm{d} r\right|_{r=r_{\max }}=0$. But the density is also positive everywhere $\rho>0$ by assumption, therefore the LHS is zero while the RHS is negative.

This is another inconsistency, the isotropic TOV cannot hold at the point $r_{\max }$.

\section{Third inconsistency}

Theorem 32. The isotropic TOV equation cannot hold in the region $r_{0}<r<r_{\max }$ where the radial pressure is positive and increasing.

Proof. Now if we look at the region between the first and second inconsistency, $r_{0}<$ $r<r_{\max }$, we can notice that the object under investigation has an increasing positive radial pressure, therefore the LHS of the TOV equation is positive in this entire region. However, because we assume $\rho>0$ which also implies that $\bar{\rho}>0$, and that there is no horizon for at least $r<r_{\max }$, the RHS of the TOV equation is negative for this entire region.

$$
-\frac{4 \pi r}{3} \frac{(\rho+p)(\bar{\rho}+3 p)}{1-2 m / r}<0
$$

This is the third inconsistency and it follows that isotropic pressure in that interval $r_{0} \leqslant r \leqslant r_{\max }$ is not able to satisfy the TOV equation, and therefore, we conclude that a static spacetime geometry can only be obtained with the introduction of tangential pressures, that is, anisotropic pressures.

\section{Fourth inconsistency}

Definition 17. Let's define $r_{g}$ the location where the quantity $\bar{\rho}+3 p$ in the isotropic TOV equation (4.19) changes sign. 
Theorem 33. The isotropic TOV equation cannot hold in the region $r_{g}<r<r_{0}$ where the radial pressure is negative and increasing and with $(\bar{\rho}+3 p)_{r_{g}}$.

Proof. The same arguments hold for a larger interval below $r_{0}$ and into the negative pressure region.

Assuming the WEC through the entire configuration implies that $\mathrm{NEC}^{17}$ holds, from which follows that

$$
\rho+p \geqslant 0 \quad \forall r: r<r_{0} .
$$

But remember that the LHS of the TOV equation is positive, therefore

$$
-\frac{4 \pi r}{3} \frac{(\rho+p)(\bar{\rho}+3 p)}{1-2 m / r}<0 \quad \Longrightarrow \quad \bar{\rho}+3 p<0 \quad \forall r<r_{0}
$$

Indeed, at the centre of the gravastar, we have

$$
(\bar{\rho}+3 p)_{c}=\rho_{c}+3 p_{c}=-2 \rho_{c}<0 .
$$

However, exactly at the point $r_{0}$, where the pressure is zero, we have

$$
(\bar{\rho}+3 p)_{r_{0}}=\bar{\rho}_{r_{0}}>0 .
$$

Therefore, it means that the term $\bar{\rho}+3 p$ changes sign somewhere in the interval $0<r<r_{0}$.

In the region $r_{g}<r<r_{0}$, we have both:

$$
\bar{\rho}+3 p>0 \text { and } \frac{\mathrm{d} p}{\mathrm{~d} r} \geqslant 0
$$

This is another inconsistency. Therefore, the pressure isotropy fails for the entire region $r_{g}<r<r_{0}$ and quite possibly fails for an even larger region.

\section{Qualitative definitions}

The following definitions relate the qualitative physics to the different regions of the gravastar described in the previous sections.

\footnotetext{
${ }^{17}$ The WEC implies the NEC, see sections 2.6.1 and 2.6.2
} 
Definition 18. The region $0<r<r_{g}$, where the physics is qualitatively similar to that of de Sitter space, will be referred as the "core". In the core, the local acceleration of equation (4.12) due to gravity is outward.

Definition 19. The region $r_{g}<r<r_{\max }$, where the physics is still definitely "unusual", will be referred as the "crust". In the crust, the local acceleration due to gravity is inward, but the pressure still rises as one moves outward.

Definition 20. The region $r_{\max }<r<R$, where the physics is still definitely "normal", will be referred as the "atmosphere". In the atmosphere, the local acceleration due to gravity is inward, and the pressure decreases as one moves outward.

Figure (4.2) presents a qualitative sketch of the previous defintions.

With these definitions, we see that pressure is guaranteed to be anisotropic throughout the "crust". Note that even if we were to dispense with the entire positive-pressure region by chopping the gravastar off at $r_{0}$, there is still an anisotropic crust in the region $\left(r_{g}, r_{0}\right]$.

In conclusion, we have shown that there are inconsistencies within the isotropic TOV equation from which follows that a static spherically symmetric object with positive density, negative central pressure and vanishing pressure at the surface cannot be supported by isotropic pressures alone.

There are no perfect fluid gravastars.

\subsubsection{A particular gravastar case study: the Schwarzschild interior solution and the Buchdahl-Bondi bound}

The Schwarzschild interior solution illustrates what can go wrong when trying to build a perfect fluid gravastar.

The Schwarzschild solution in standard coordinates $(t, r, \theta, \phi)$ is given by equation (2.31). But the general Schwarzschild interior solution for values of $r$ smaller than the Schwarzschild radius $R_{\text {Schwarzschild }}$ is given by: 


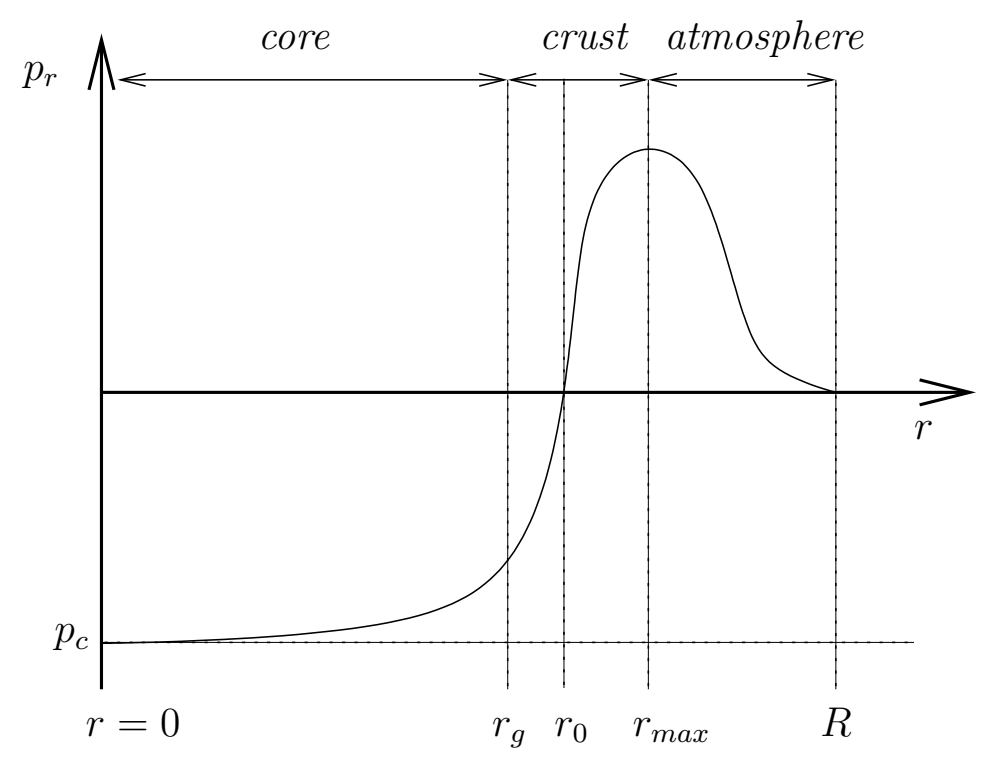

Figure 4.2: Qualitative sketch of gravastar labelling the "core", "crust", and "atmosphere".

$$
d s^{2}=-\left(A+B \sqrt{1-\frac{2 m_{*}(r)}{r}}\right)^{2} d t^{2}+\left(\frac{1}{1-\frac{2 m_{*}(r)}{r}}\right) d r^{2}+r^{2} d \Omega^{2}
$$

where $d \Omega^{2}=d \theta^{2}+\sin ^{2} \theta d \phi^{2}$ and $m_{*}(r)=M(r / R)^{3}$. This general solution describes static spherically symmetric stars with constant density independent of $r$ from the centre to the surface of the star.

Using boundary conditions, we can determine $A$ and $B$ as functions of the surface radius $R$ and the total mass $M$ of the star. The density can be calculated with the $G_{t t}$ term of the Einstein tensor and $g_{t t}$ part of the metric (A.7) due to the field equations (2.15):

$$
\begin{aligned}
8 \pi \rho_{*} g_{t t} & =G_{t t} \\
\Longrightarrow \quad \rho_{*} & =\frac{3 M}{4 \pi R^{3}} .
\end{aligned}
$$


The value of the density is constant and depends only on $M$ and $R$ that are fixed for a specific star.

The radial pressure can also be calculated exactly with the field equations in the $\mathrm{rr}$ components as a function of $A$ and $B$ from the general metric. However, the expression of $p^{*}$ is quite complicated and messy, but the important feature is, that the surface radius $R$ can be deduced as a function of $A$ and $B$ from the boundary condition:

$$
\text { at } R_{\text {surface }} \quad p^{*}(R)=0 \quad \Longrightarrow \quad f_{1}(A, B, R, M)=0 .
$$

Furthermore, the total mass of the star is:

$$
M=4 \pi \int_{r=0}^{r=R(A, B, R, M)} \rho^{*} r^{2} d r \quad \Longrightarrow \quad f_{2}(A, B, R, M)=0 .
$$

Therefore, solving the following system (4.43) of two equations for $A$ and $B$

$$
\left\{\begin{array}{l}
f_{1}(A, B, R, M)=0 \\
f_{2}(A, B, R, M)=0
\end{array}\right.
$$

leads to $A(R, M)$ and $B(R, M)$ which determines the metric of the Schwarzschild interior solution entirely as a function of the total mass $M$ and the surface radius $R$. Indeed, it can be found that:

$$
\left\{\begin{array}{l}
A=\sqrt{1-\frac{2 M}{R}} \\
B=-\frac{1}{3} .
\end{array}\right.
$$

In this case the metric (A.7) becomes:

$$
d s^{2}=-\left(\sqrt{1-\frac{2 M}{R}}-\frac{1}{3} \sqrt{1-\frac{2 m_{*}(r)}{r}}\right)^{2} d t^{2}+\left(\frac{1}{1-\frac{2 m_{*}(r)}{r}}\right) d r^{2}+r^{2} d \Omega^{2}
$$

where $m_{*}(r)=M(r / R)^{3}$.

In 1916, Schwarzschild integrated exactly by hand the isotropic equation (4.19) yeilding

$$
p_{*}(r)=\rho_{*} \frac{\sqrt{1-2 m_{*}(r) / r}-\sqrt{1-2 M / R}}{3 \sqrt{1-2 M / R}-\sqrt{1-2 m_{*}(r) / r}}
$$

where $M$ and $R$ are the total mass and surface radius. Note that the denominator of the pressure $p_{*}$ is exactly $3 g_{t t}$ from the $t t$-component of the metric (4.45). 


\section{Central pressure $p_{c}^{*}$ and the Buchdahl-Bondi bound}

The central pressure required for equilibrium of a uniform density star is

$$
p_{c}^{*}=\rho_{*} \frac{1-\sqrt{1-2 M / R}}{3 \sqrt{1-2 M / R}-1}
$$

For $R \gg M$, equation (4.47) reduces to the Newtonian value $(4.49)^{18} 19$ :

$$
p_{c}^{*}=\left(\frac{\pi}{6}\right)^{\frac{1}{3}} M^{\frac{2}{3}} \rho_{*}^{\frac{4}{3}}
$$

The sign of the central pressure $p_{c}^{*}$ is determined by specific conditions only on the denominator as the numerator is always positive:

$$
1-\sqrt{1-2 M / R}>0 \quad \Longleftrightarrow \quad 0>-\frac{2 M}{R} \quad \text { (always true). }
$$

The central pressure $p_{c}^{*}$ in the gravastar becomes infinite when

$$
3 \sqrt{1-2 M / R}=1 \quad \Longrightarrow \quad \frac{2 M}{R}=\frac{8}{9},
$$

and therefore, $p_{c}^{*}>0$ when

$$
3 \sqrt{1-2 M / R}>1 \quad \Longleftrightarrow \quad \frac{2 M}{R}<\frac{8}{9} .
$$

This result on the compactness, $\chi=2 M / R$, is known as the Buchdahl-Bondi bound: the compactness of a static spherically symmetric fluid $\chi$ is bounded above by $8 / 9$. This is quite significant as it shows that a star cannot get arbitrarily close to forming a black hole $(\chi=1)$.

However, in our particular gravastar model, we deal with negative central pressures $p_{c}^{*}<0$, and this implies that we have the relation:

$$
p_{c}^{*}<0 \quad \Longleftrightarrow \quad \frac{8}{9}<\frac{2 M}{R}<1
$$

\footnotetext{
${ }^{18}$ Note that the isotropic TOV equation in a Newtonian star is

$$
\frac{d p}{d r}=-g(r) \rho(r)=-\frac{m(r)}{r^{2}} \rho(r)=\frac{4}{3} \pi r \bar{\rho} .
$$
}

${ }^{19}$ Value given in [79] 
Of course, while this is a perfectly sensible solution in the mathematical sense one would generally rule it out physically because of the negative central pressure. If we leave our prejudice against negative pressure aside, adopting the gravastar philosophy, we will find some interesting results coming from the choice of (4.53).

\section{First order pole}

Theorem 34. For a Schwarzschild interior solution, with constant positive density $\rho_{*}>0$, and a negative central pressure $p_{c}^{*}<0$, the pressure profile given by equation (4.46) will have a first order pole at

$$
r_{\text {pole }}=3 R \sqrt{1-\frac{8 / 9}{2 M / R}} \quad \text { and furthermore } \quad r_{\text {pole }}<R
$$

Note that Theorem 34 assumes constant positive density $\rho_{*}>0$ and a central negative pressure $p_{c}^{*}<0$, but it does not assume that at the centre $p_{c}^{*}=-\rho_{*}$. As a matter of fact, the only way to obtain exactly $p_{c}^{*}=-\rho_{*}$ at the centre is if $R=2 M$ that is if there is a Schwarzschild radius. Indeed, $p_{c}^{*}=-\rho_{*}$ if and only if:

$$
\frac{p_{c}^{*}}{\rho_{*}}=\frac{1-\sqrt{1-2 M / R}}{3 \sqrt{1-2 M / R}-1}=-1,
$$

that is, if,

$$
2 \sqrt{1-2 M / R}=0
$$

finally, if

$$
R=2 M \text {. }
$$

Proof. From the pressure profile given in equation (4.46), there is a pole when its denominator cancels out or, in this case, when the $t$-component of the metric (4.45) is zero.

$$
g_{t t}=0 \quad \Longrightarrow \quad 3 \sqrt{1-2 \frac{M}{R}}-\sqrt{1-2 m_{*}(r) / r}=0
$$


where $m_{*}(r)=M(r / R)^{3}$. We can extract the radius $r$ from this relation and obtain a value for $r$ at the pole.

$$
\begin{aligned}
\sqrt{1-2 \frac{M r^{2}}{R^{3}}} & =3 \sqrt{1-2 \frac{M}{R}} \\
\Longrightarrow \quad 1-2 \frac{M r^{2}}{R^{3}} & =9\left(1-2 \frac{M}{R}\right) \\
\Longrightarrow \quad-2 \frac{M r^{2}}{R^{3}} & =\left(8-9 \times 2 \frac{M}{R}\right) \\
r^{2} & =9 R^{2}\left(1-\frac{8 / 9}{2 M / R}\right) \\
\Longrightarrow \quad r_{\text {pole }} & =3 R \sqrt{1-\frac{8 / 9}{2 M / R}}
\end{aligned}
$$

Now we can see what the conditions to have the upper bound $R$ on $r_{\text {pole }}$ are:

$$
\begin{aligned}
3 R \sqrt{1-\frac{8 / 9}{2 M / R}} & <R \\
\Longrightarrow \quad 9 R^{2}\left(1-\frac{8 / 9}{2 M / R}\right) & <R^{2} \\
\Longrightarrow \quad\left(8 / 9-\frac{8 / 9}{2 M / R}\right) & <0 \\
\Longrightarrow \quad & <1 \text { true for no event horizon }
\end{aligned}
$$

Therefore, for a Schwarzschild interior solution, with negative central pressure and constant positive density, that has no event horizon, there exists a pole such as

$$
r_{\text {pole }}<R
$$

where $R$ is the surface.

Note that as $2 M / R$ goes from $8 / 9$ to 1 , the position of this pole moves from the centre of the star to the surface of the star. The situation is qualitatively sketched in figure 4.3 . 


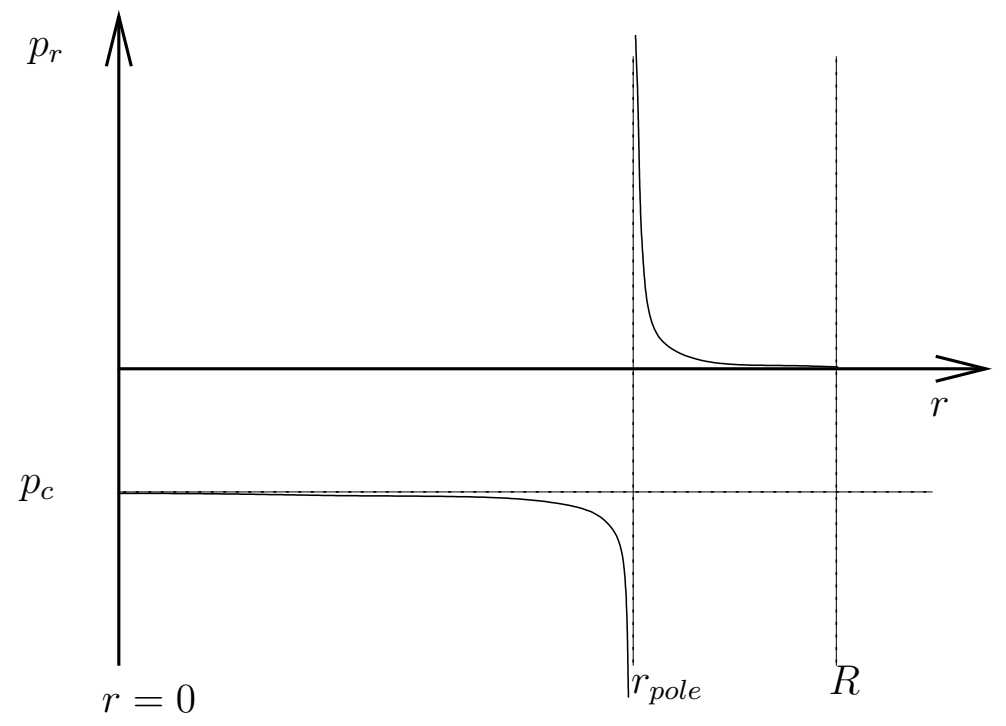

Figure 4.3: Qualitative sketch of pressure pole in the interior Schwarzschild solution for $2 M / R>8 / 9$.

Theorem 35. The NEC must be violated sufficiently close to the pole at $r_{\text {pole }}=$ $3 R \sqrt{1-\frac{8 / 9}{2 M / R}}$.

Proof. The compactness is bounded $8 / 9<2 M / R<1$ which implies that the denominator term in equation (4.46) is negative, and therefore, that the pressure $p^{*}$ tends to $-\infty$ as $r$ approaches $r_{\text {pole }}$.

The NEC holds, in the vicinity of the pole, if and only if:

$$
\rho_{*}+p^{*} \geqslant 0
$$

However, near the pole,

$$
\lim _{r \longrightarrow r_{\mathrm{pole}}}\left(\rho_{*}+p^{*}\right)=-\infty
$$

Therefore, the NEC must be violated sufficiently close to the pole. 


\section{Curvature singularity}

We are going to see now that Schwarzschild interior solution gravastars are clearly unphysical, even if one is willing to accept negative pressures and even violations of the NEC. It is unphysical because the pressure pole implies a curvature singularity. With the metric given by equation (4.45), we can calculate the Riemann tensor in an orthonormal basis $R_{\hat{a} \hat{b} \hat{c} \hat{d}}$. In particular, for isotropic pressures, we know that,

$$
\begin{aligned}
R_{\hat{t} \hat{r} \hat{r} \hat{r}} & =-\frac{R_{t r t r}}{g_{t t} g_{r r}}=\frac{4 \pi}{3}(\rho+3 p) \\
R_{\hat{t} \hat{\theta} \hat{\theta} \hat{\theta}} & =-\frac{R_{t \theta t \theta}}{g_{t t} g_{\theta \theta}}=\frac{4 \pi}{3}(\rho+3 p) \\
R_{\hat{t} \hat{t} \hat{\phi} \hat{\phi}} & =-\frac{R_{t \phi t \phi}}{g_{t t} g_{\phi \phi}}=\frac{4 \pi}{3}(\rho+3 p) .
\end{aligned}
$$

Now, we know that those specific terms of the Riemann tensor will have a problem at $r=r_{\text {pole }}$. As shown previously for the pressure pole, the term $g_{t t}$ is zero at $r=r_{\text {pole }}$ and

$$
g_{t t}=O\left(\left[r-r_{\text {pole }}\right]^{2}\right) \text {, }
$$

whereas $g_{r r}$ is finite at $r=r_{\text {pole }}$ and

$$
g_{r r}\left(r_{\text {pole }}\right)=\frac{1}{9(1-2 M / R)}>0 .
$$

Concerning the trtr-term of the Riemann tensor, we have

$$
\begin{aligned}
\lim _{r \rightarrow r_{\mathrm{pole}}} R_{\text {trtr }} & =0 \\
\lim _{r \rightarrow r_{\mathrm{pole}}} \frac{d R_{\text {trtr }}}{d r} & =\frac{3}{81 R^{3}}\left(\frac{2 M}{R}\right)^{2} \frac{\left(1-\frac{8 / 9}{2 M / R}\right)^{1 / 2}}{(1-2 M / R)}
\end{aligned}
$$

Hence, we have for the trtr-term of the Riemann tensor at the pole:

$$
\lim _{r \rightarrow r_{\text {pole }}} R_{t r t r}=O\left(\left[r-r_{\text {pole }}\right]\right) .
$$

Therefore, the $\hat{t} \hat{r} \hat{t} \hat{r}$-term of the Riemann tensor becomes infinite at $r=r_{\text {pole }}$, as $g_{t t}$ becomes the dominant term:

$$
\lim _{r \rightarrow r_{\text {pole }}} R_{\hat{t} \hat{r} \hat{r} \hat{r}}=\infty .
$$


On a similar way, we have $g_{\theta \theta}$ and $g_{\phi \phi}$ finite at the pole, and

$$
\begin{aligned}
& g_{\theta \theta}\left(r_{\text {pole }}\right)=9 R^{2}\left(1-\frac{8 / 9}{2 M / R}\right)>0, \\
& g_{\phi \phi}\left(r_{\text {pole }}\right)=9 \sin ^{2} \theta R^{2}\left(1-\frac{8 / 9}{2 M / R}\right)>0 .
\end{aligned}
$$

Concerning the $t \theta t \theta$ and $t \phi t \phi$ terms of the Riemann tensor, we have,

$$
\begin{aligned}
\lim _{r \rightarrow r_{\text {pole }}} R_{t \theta t \theta} & =0 \\
\lim _{r \rightarrow r_{\text {pole }}} \frac{d R_{t \theta t \theta}}{d r} & =\frac{3}{R}\left(\frac{2 M}{R}\right)^{2}\left(1-\frac{8 / 9}{2 M / R}\right)^{1 / 2}, \\
\lim _{r \rightarrow r_{\text {pole }}} R_{t \phi t \phi} & =0 \\
\lim _{r \rightarrow r_{\text {pole }}} \frac{d R_{t \phi t \phi}}{d r} & =\frac{3}{R}\left(\frac{2 M}{R}\right)^{2}\left(1-\frac{8 / 9}{2 M / R}\right)^{1 / 2} \sin ^{2}(\theta) .
\end{aligned}
$$

Hence, we have for the $t \theta t \theta$ and $t \phi t \phi$ terms of the Riemann tensor at the pole:

$$
\begin{aligned}
& \lim _{r \rightarrow r_{\text {pole }}} R_{t \theta t \theta}=O\left(\left[r-r_{\text {pole }}\right]\right), \\
& \lim _{r \rightarrow r_{\text {pole }}} R_{t \phi t \phi}=O\left(\left[r-r_{\text {pole }}\right]\right) .
\end{aligned}
$$

Therefore, the $\hat{t} \hat{\theta} \hat{t} \hat{\theta}$-term and the $\hat{t} \hat{\phi} \hat{t} \hat{\phi}$-term of the Riemann tensor become infinite at $r=r_{\text {pole }}$, as $g_{t t}$ becomes the dominant term:

$$
\begin{aligned}
& \lim _{r \rightarrow r_{\text {pole }}} R_{\hat{t} \hat{\theta} \hat{t} \hat{\theta}}=\infty, \\
& \lim _{r \rightarrow r_{\text {pole }}} R_{\hat{t} \hat{\phi} \hat{t} \hat{\phi}}=\infty .
\end{aligned}
$$

However, the rest of the non-zero orthonormal components of the Riemann tensor are finite,

$$
\begin{aligned}
R_{\hat{r} \hat{\theta} \hat{r} \hat{\theta}} & =\frac{2 M}{R^{3}} \\
R_{\hat{r} \hat{\phi} \hat{r} \hat{\phi}} & =\frac{2 M}{R^{3}} \\
R_{\hat{\theta} \hat{\phi} \hat{\theta} \hat{\phi}} & =\frac{2 M}{R^{3}} .
\end{aligned}
$$


To conclude, we have shown that 3 orthonormal components of the Riemann tensor $\left(R_{\hat{t} \hat{r} \hat{t} \hat{r}}\right.$ (4.79), $R_{\hat{t} \hat{\theta} \hat{t} \hat{\theta}}$ (4.88), $\left.R_{\hat{\theta} \hat{\phi} \hat{\theta} \hat{\phi}}(4.89)\right)$ are infinite at $r=r_{\text {pole }}$, and therefore, the pressure pole implies a naked singularity.

The reason why we mention this specific example is because we shall soon see that this "pressure pole" behaviour is generic. Continuous solutions with isotropic pressure are not possible ${ }^{20}$.

\subsubsection{Arbitrary gravastar case study: the fate of a negative pressure perfect fluid sphere}

In this section, we consider an arbitrary gravastar as defined in section (4.2), and we will show that if the gravastar configuration is perfect fluid, the same problems as those encountered in the previous section (4.3.2) will arise in this more general case.

For any arbitrary gravastar that is static spherically symmetric, remember that the geometry is given by

$$
d s^{2}=-\exp \left(2 \int_{r}^{\infty} g(\tilde{r}) d \tilde{r}\right) d t^{2}+\frac{d r^{2}}{\left(1-\frac{2 m(r)}{r}\right)}+r^{2} d \Omega^{2}
$$

where

$$
g(r)=\frac{m(r)+4 \pi p_{r}(r) r^{3}}{r^{2}\left(1-\frac{2 m(r)}{r}\right)} .
$$

Theorem 36. If an arbitrary gravastar is perfect fluid and finite, and if we permit the violation of the NEC, then there exists a pressure pole of order 1 and it is the only way the TOV equation can be satisfied.

Proof. Using equation (4.13) that relates the density $\rho$ to the mass $m(r)$, we can obtain an expression for the compactness $\chi=2 m(r) / r$ which satisfies:

$$
\left[\frac{2 m(r)}{r}\right]^{\prime}=8 \pi \rho r-\frac{2 m}{r^{2}}=\frac{8 \pi r}{3}[3 \rho-\bar{\rho}]=\frac{8 \pi r}{3}[3(\rho+p)-(\bar{\rho}+3 p)] .
$$

\footnotetext{
${ }^{20}$ Consider for example [10], where a perfect fluid Chaplygin gas $\rho \propto 1 / p$ is considered. The surface of their configuration occurs at $\rho=0$ where $p=-\infty$, at least as one approaches the surface from below. So the surface of their configuration is a naked singularity, in agreement with the observations above.
} 
But the first term on the RHS is non-negative by the NEC, while the second term is by definition negative on $\left[0, r_{g}\right)$, so the compactness $2 m(r) / r$ is monotone increasing on the range $\left[0, r_{g}\right)$. This is somewhat unusual, while the compactness of a normal perfect fluid star tends to increase as one moves outwards, in a normal star it also can be subject to oscillations that make the overall picture quite subtle [42, 78].

Let's consider now a perfect fluid sphere with negative central pressure that satisfies the NEC. Since we have already seen that isotropy is violated on $\left(r_{g}, r_{\max }\right)$, the only way we can maintain the perfect fluid nature of the sphere is if $r_{g} \rightarrow \infty$, which also implies that $r_{0} \rightarrow \infty$. But since we do not want a horizon to form, the compactness must be bounded above by unity. But we have just shown the compactness is monotonic and therefore,

$$
\lim _{r \rightarrow \infty} \frac{2 m(r)}{r}=\chi_{*} ; \quad \chi_{*} \in(0,1] .
$$

So not only does a NEC-satisfying perfect-fluid gravastar expand to infinite volume, it also has infinite mass.

To avoid the physical size of the gravastar blowing up to $r_{0} \rightarrow \infty$, our options now are rather limited: we could permit the development of a horizon at finite $r$, which defeats the whole point of the exercise, or we could permit something even worse.

\section{First order pressure pole}

If we permit NEC violations then it is possible to arrange for the development of a pressure pole at finite $r<r_{0}$. Let's see how this is possible by first considering the following definition.

Definition 21. Set

$$
p(r) \approx \frac{\Gamma}{r-r_{p}}
$$

in the vicinity of the pressure pole of order 1 at $r_{p}$ where $\Gamma$ is a positive constant.

This definition is compatible with the isotropic TOV equation. First for $r<r_{p}$ the pressure is negative, and for $r>r_{p}$ the pressure is positive, which is appropriate for a 
modified gravastar model. Secondly

$$
p^{\prime} \approx \frac{-\Gamma}{\left(r-r_{p}\right)^{2}}<0 .
$$

Third, assuming $\rho$ remains finite, close to the pole $\left(r \approx r_{p}\right)$ we have

$$
\begin{aligned}
-\frac{(\rho+p)\left(m+4 \pi p r^{3}\right)}{r^{2}(1-2 m / r)} & \approx-\frac{\left[\rho+\Gamma /\left(r-r_{p}\right)\right]\left[m+4 \pi r^{3} \Gamma /\left(r-r_{p}\right)\right]}{r^{2}(1-2 m(r) / r)} \\
& \approx-\frac{\left[\Gamma /\left(r-r_{p}\right)\right]\left[4 \pi r^{3} \Gamma /\left(r-r_{p}\right)\right]}{r^{2}(1-2 m(r) / r)} \\
& \approx-\frac{4 \pi r_{p} \Gamma^{2}}{\left(1-2 m\left(r_{p}\right) / r_{p}\right)} \frac{1}{\left(r-r_{p}\right)^{2}}<0 .
\end{aligned}
$$

So the TOV equation can be satisfied in the vicinity of the pole provided

$$
-\frac{(\rho+p)\left(m+4 \pi p r^{3}\right)}{r^{2}(1-2 m / r)} \approx p_{r}^{\prime} \quad \text { at } r=r_{g}
$$

that is provided we set

$$
\Gamma=\frac{1-2 m\left(r_{p}\right) / r_{p}}{4 \pi r_{p}}>0 .
$$

That is, if the gravastar configuration is perfect fluid and finite in extent, then this pole is the only way the TOV equation can be satisfied.

\section{$n^{\text {th }}$ order pressure pole}

Now we can look at higher order pole and see if they also have the nice property of being compatible with the isotropic TOV equation.

Definition 22. Set

$$
p(r) \approx \frac{\Gamma}{\left(r-r_{p}\right)^{n}},
$$

in the vicinity of the pressure pole of order $n>1$ at $r_{p}$ where $\Gamma$ is a positive constant.

This definition is compatible with the isotropic TOV equation for odd value of $n$ only. First for $r<r_{p}$ the pressure is negative if $n$ is odd, and for $r>r_{p}$ the pressure 
is positive if $n$ is odd. However, this definition is not compatible with the isotropic TOV equation for even values of $n$. Secondly

$$
p^{\prime} \approx \frac{-n \Gamma}{\left(r-r_{p}\right)^{(n+1)}}<0
$$

Third, assuming $\rho$ remains finite, close to the pole $\left(r \approx r_{p}\right)$ we have

$$
\begin{aligned}
-\frac{(\rho+p)\left(m+4 \pi p r^{3}\right)}{r^{2}(1-2 m / r)} & \approx-\frac{\left[\rho+\Gamma /\left(r-r_{p}\right)^{n}\right]\left[m+4 \pi r^{3} \Gamma /\left(r-r_{p}\right)^{n}\right]}{r^{2}(1-2 m(r) / r)} \\
& \approx-\frac{\left[\Gamma /\left(r-r_{p}\right)^{n}\right]\left[4 \pi r^{3} \Gamma /\left(r-r_{p}\right)^{n}\right]}{r^{2}(1-2 m(r) / r)} \\
& \approx-\frac{4 \pi r_{p} \Gamma^{2}}{\left(1-2 m\left(r_{p}\right) / r_{p}\right)} \frac{1}{\left(r-r_{p}\right)^{2 n}}<0
\end{aligned}
$$

So the TOV equation can be satisfied in the vicinity of the pole provided

$$
-\frac{(\rho+p)\left(m+4 \pi p r^{3}\right)}{r^{2}(1-2 m / r)} \approx p_{r}^{\prime} \quad \text { at } r=r_{g}
$$

that is provided we set

$$
\Gamma=\frac{1-2 m\left(r_{p}\right) / r_{p}}{4 \pi r_{p}} n\left(r-r_{p}\right)^{n-1} \geqslant 0
$$

First, equation (4.110) is in total contradiction with the Definition 22 of $\Gamma$ that is supposed to be a constant. Secondly, it shows that the isotropic TOV equation cannot hold at the pressure pole of order $n$.

Therefore, higher-order poles do not even have this nice property of being compatible with the isotropic TOV equation.

Theorem 37. An arbitrary finite gravastar with a perfect fluid configuration that has a pressure pole leads to a curvature singularity.

Proof. For any isotropic static spherically symmetric spacetime the orthonormal com- 
ponents of the Riemann tensor are (see, for instance, [74, p 110]):

$$
\begin{aligned}
R_{\hat{t} \hat{r} \hat{r}} & =\frac{4 \pi}{3}[3(\rho+p)-2 \bar{\rho}] \\
R_{\hat{t} \hat{\theta} \hat{\theta} \hat{\theta}} & =\frac{4 \pi}{3}[3 p+\bar{\rho}] ; \\
R_{\hat{r} \hat{\theta} \hat{r} \hat{\theta}} & =\frac{4 \pi}{3}[3 \rho-\bar{\rho}] ; \\
R_{\hat{\theta} \hat{\theta} \hat{\theta} \hat{\phi}} & =\frac{4 \pi}{3}[2 \bar{\rho}] .
\end{aligned}
$$

It is clear that if the pressure has a pole of order $n \geqslant 1$, then, providing $\rho$ remains finite as per assumption, as $R_{\hat{t} \hat{r} \hat{t} \hat{r}}$ and $R_{\hat{t} \hat{\theta} \hat{t} \hat{\theta}}$ depend on $p$, they will also have a pole, and consequently, a naked singularity will arise.

Even if the isotropic TOV equation holds with pressure pole in the gravastar configuration, this situation is physically inappropriate as the pressure pole introduces a naked singularity. The physically correct deduction form this analysis is that gravastarlike objects must violate pressure isotropy.

\section{Summary}

In this section, we have shown that a static spherically symmetric object with positive density, negative central pressure, and vanishing pressure at the surface, (which are the defining features of a gravastar), cannot be supported by isotropic pressure alone without a pole in the pressure. Despite the fact that the presence of a simple pole in the pressure is compatible with the isotropic TOV equation, we must reiterate that such a pressure pole is unphysical because it is a naked singularity. Therefore, gravastar-like objects must violate pressure isotropy.

\subsection{Gravastars with anisotropy}

In section (4.3), we have demonstrated that gravastars cannot have isotropic pressure alone, and that, as a consequence, anisotropic pressure must be part of the configuration or at least in some parts of it. In this section, we are going to see when 
the anisotropy for the pressure is necessary for the TOV equation to hold, and what specific features the equation of state must have.

\subsubsection{Bounds on the anisotropy pressure}

Once we accept that perfect fluid spheres are not what we are looking for to model gravastars, one might wonder what happens to the Buchdahl-Bondi bound for isotropic fluid spheres. It has been shown that for $\rho^{\prime}<0$ and $p_{t} \leq p_{r}$ the $8 / 9$ bound still holds ${ }^{21}$. However if the transverse stress is allowed to exceed the radial stress, (e.g., $p_{t}>p_{r}$ ), then the upper limit shifts to $2 M / R<\kappa \leq 1$, where $\kappa$ depends on the magnitude of the maximal stress anisotropy $[41]^{22}$.

Remember that in section 4.3.1 we defined the crust of the gravastar as the region $r_{g}<r<r_{\max }$, where the physics is still definitely "unusual", and where the local acceleration due to gravity is inward, but the pressure still rises as one moves outward. The dimensionless anisotropy parameter $\Delta$ is a function of the radial pressure, tangential pressure and positive density:

$$
\Delta=\frac{p_{t}-p_{r}}{\rho}=\frac{r}{2}\left[\frac{p_{r}^{\prime}}{\rho}+\left(1+\frac{p_{r}}{\rho}\right) g\right] .
$$

Theorem 38. In the crust of a gravastar, the compactness is not limited to the Buchdahl-Bondi bound, but only by the magnitude of the maximal pressure anisotropy and the regularity of the metric:

$$
\Delta \geq \frac{1}{4} \frac{2 m / r}{1-2 m / r}>0 \quad r_{g}<r<r_{0}
$$

and

$$
0 \leq \frac{r p_{r}^{\prime}}{2 \rho}<\Delta<\frac{r p_{r}^{\prime}}{2 \rho}+\frac{1}{4} \frac{2 m / r}{1-2 m / r} \quad r_{0}<r<r_{\max }
$$

Furthermore, the tangential pressure is greater than the radial pressure $p_{t}>p_{r}$ for $r_{g}<r<r_{\max }$.

\footnotetext{
${ }^{21}$ See section $(4.3 .2)$

${ }^{22}$ For more related work on anisotropic stars see $[45,44,46]$
} 
Proof. In section 4.3.1, we have determined that anisotropy is necessary in the crust $^{23}$, that is, when $r_{g}<r<r_{\max }$. We can now find bounds on $\Delta$ in that interval by inserting the definition of the gravitational acceleration $g(r)$ into equation (4.115). We get,

$$
\Delta=\frac{r}{2}\left[\frac{p_{r}^{\prime}}{\rho}+\left(1+\frac{p_{r}}{\rho}\right) \frac{m+4 \pi p_{r} r^{3}}{r^{2}\left[1-\frac{2 m}{r}\right]}\right] .
$$

Now we know that for the interval $r \in\left[r_{0}, r_{\max }\right]$, the radial pressure is positive $p_{r} \geq 0$ and increasing so $p_{r}^{\prime} \geq 0$. Therefore, we can write the inequality,

$$
\begin{aligned}
& \Delta \geq \frac{r}{2}\left(1+\frac{p_{r}}{\rho}\right) \frac{m}{r^{2}[1-2 m / r]}>0 \\
& \Delta \geq \frac{r}{2} \frac{m}{r^{2}[1-2 m / r]}>0,
\end{aligned}
$$

that leads to the simple lower bound:

$$
\Delta \geq \frac{1}{4} \frac{2 m / r}{1-2 m / r}>0
$$

The inequality on $\Delta(4.121)$, for the interval $r \in\left[r_{0}, r_{\max }\right]$, shows that it is positive, consequently, in this region, we have $p_{t}>p_{r}>0$.

Now let's see what happens for the interval $r \in\left(r_{g}, r_{0}\right)$. We know that in this area, the radial pressure is negative $p_{r}<0$, but it is increasing and $\mathrm{d} p_{r} / \mathrm{d} r>0$. From these conditions on the radial pressure and its derivative, we can write,

$$
\begin{aligned}
\Delta & \leqslant \frac{r}{2}\left(\frac{p_{r}^{\prime}}{\rho}+1\right) \frac{m+4 \pi p_{r} r^{3}}{r^{2}(1-2 m / r)} \\
\Delta & \leqslant \frac{r p_{r}^{\prime}}{2 \rho}+\frac{r}{2} \frac{m}{r^{2}[1-2 m / r]} \\
\Delta & \leqslant \frac{r p_{r}^{\prime}}{2 \rho}+\frac{1}{4} \frac{2 m / r}{1-2 m / r} .
\end{aligned}
$$

Remember that we assume that the NEC holds in the configuration of the gravastar.

\footnotetext{
${ }^{23}$ Isotropy possibly fails for an even larger region.
} 
This leads to

$$
\begin{aligned}
\rho+p_{r} & >0 \\
p_{r} & >-\rho \\
\frac{p_{r}}{\rho} & >-1 \\
\left(1+\frac{p_{r}}{\rho}\right) & >0 .
\end{aligned}
$$

This last inequality (4.128), allows us to write a lower bound on $\Delta$ :

$$
\left(\frac{p_{r}^{\prime}}{\rho}+1\right) \frac{m+4 \pi p_{r} r^{3}}{r^{2}(1-2 m / r)} \quad \Longrightarrow \quad \Delta \geqslant \frac{r p_{r}^{\prime}}{2 \rho} \geqslant 0
$$

Consequently, for the interval $r \in\left(r_{g}, r_{0}\right)$, we find the weaker bounds

$$
0 \leq \frac{r p_{r}^{\prime}}{2 \rho}<\Delta<\frac{r p_{r}^{\prime}}{2 \rho}+\frac{1}{4} \frac{2 m / r}{1-2 m / r}
$$

Theorem 39. In the interval $\left[r_{0}, r_{\max }\right]$, if the DEC holds, then

$$
\mathrm{DEC} \quad \Longrightarrow \quad \Delta \leqslant 1 \text {. }
$$

Proof. For the first inequality on $\Delta$ (4.121), in the region $r \in\left[r_{0}, r_{\max }\right]$, the DEC is satisfied if

$$
\begin{array}{r}
\rho \geqslant 0, \\
-\rho \leqslant p_{r} \leqslant \rho, \\
-\rho \leqslant p_{t} \leqslant \rho .
\end{array}
$$

This leads to the more useful inequalities,

$$
\begin{aligned}
& \left\|\frac{p_{r}}{\rho}\right\| \leqslant 1, \\
& \left\|\frac{p_{t}}{\rho}\right\| \leqslant 1 .
\end{aligned}
$$


But because the radial pressure is positive for the interval $\left[r_{0}, r_{\max }\right]$, we can write,

$$
\Delta=\frac{p_{t}-p_{r}}{\rho} \leqslant \frac{p_{t}}{\rho} \leqslant 1
$$

And therefore, if the DEC is to be satisfied, it implies the following upper bound on $\Delta$ :

$$
\mathrm{DEC} \quad \Longrightarrow \quad \Delta \leqslant 1 \text {. }
$$

Theorem 40. Any gravastar that is sufficiently close to forming a horizon, that is $2 \mathrm{~m} / \mathrm{r}>4 / 5$, will violate the DEC in its "crust".

Proof. If the DEC is to be satisfied we must at the very least have $\Delta \leq 1$. Therefore, when the DEC holds, we have

$$
\begin{aligned}
& 1 \geqslant \Delta \geq \frac{1}{4} \frac{2 m / r}{1-2 m / r}>0 \\
& 1 \geqslant \frac{1}{4} \frac{2 m / r}{1-2 m / r}>0 \\
& 4 \geqslant 5 \frac{2 m}{r} \\
& \frac{4}{5} \geqslant \frac{2 m}{r}
\end{aligned}
$$

Hence, the DEC is guaranteed to be violated whenever $2 \mathrm{~m} / r>4 / 5$. If the gravastar is sufficiently close to forming a horizon, in the sense that $2 \mathrm{~m} / r>4 / 5$ somewhere in the range $\left[r_{0}, r_{\max }\right]$, then the DEC must also be violated at this point. Even if we were to discard the entire positive-pressure region $\left(r_{0}, R\right)$, we can nevertheless still apply this bound at $r_{0}$ itself: if $2 m\left(r_{0}\right) / r_{0}>4 / 5$ then the DEC is violated at $r_{0}$. Consequently, any gravastar that is sufficiently close to forming a horizon will violate the DEC in its "crust".

\subsubsection{Minimizing the anisotropy}

We have shown that the anisotropy is necessary for gravastar-like objects in their "crust", where the pressure is increasing as one moves outwards and the local force 
of gravity is inwards. In this section, we attempt to minimize the region over which anisotropy is present.

Remember that he dimensionless anisotropy parameter $\Delta$ is deduced from the anisotropy TOV equation (4.21), and gives,

$$
\Delta=\frac{p_{t}-p_{r}}{\rho}=\frac{r}{2}\left[\frac{p_{r}^{\prime}}{\rho}+\left(1+\frac{p_{r}}{\rho}\right) g\right] .
$$

\section{What happens at $r_{g}$ ?}

At the point $r_{g}$, we can write (since $g\left(r_{g}\right)=0$ by definition 17 )

$$
\Delta\left(r_{g}\right)=\frac{r_{g} p_{r}^{\prime}\left(r_{g}\right)}{2 \rho} \geqslant 0
$$

To confine the anisotropy to the smallest interval possible, we have to set

$$
p_{r}^{\prime}\left(r_{g}\right)=0,
$$

which corresponds to an inflexion point for the radial pressure.

What happens at $r_{0}$ ?

At the point $r_{0}$, the radial pressure is zero, and we can write,

$$
\Delta\left(r_{0}\right)=\frac{r_{0} p_{r}^{\prime}}{2 \rho}+\frac{1}{4} \frac{2 m / r_{0}}{1-2 m / r_{0}}>0 .
$$

Hence, at the point of zero radial pressure, the anisotropy cannot vanish.

What happens at $r_{\max }$ ?

At the point $r_{\max }$, the radial pressure reaches its maximum value. If we take the limit from below, we can write,

$$
\Delta\left(r_{\max }^{-}\right)=\frac{1}{4} \frac{2 m / r_{\max }}{1-2 m / r_{\max }}\left(1+\frac{p_{r}}{\rho}\right)\left(1+\frac{4 \pi p_{r} r_{\max }^{3}}{m}\right)>0 .
$$


Remember that the anisotropy is not necessary beyond the peak $\forall r>r_{\max }$ (in the atmosphere), and therefore, it is possible to arrange $\Delta=0$. To confine the anisotropy to the smallest interval possible, we have to set

$$
\Delta\left(r \rightarrow r_{\max }^{+}\right)=0
$$

However, this leads to a discontinuity in the derivative of the radial pressure $p_{r}^{\prime}$ and the anisotropy parameter $\Delta$, as well as a "kink" in the pressure profile $p_{r}$ at $r_{\max }$.

From the TOV equation (4.21), we can deduce the limit from above for the pressure derivative,

$$
p_{r}^{\prime}\left(r_{\max }^{+}\right)=-\left(\rho+p_{r}\right)\left(\frac{m+4 \pi p_{r} r_{\max }^{3}}{r^{2}\left(1-2 m / r_{\max }\right)}\right) .
$$

$\Delta$ at $r_{\max }^{-}$can be rewritten as

$$
\Delta\left(r_{\max }^{-}\right)=\frac{1}{2}\left(1+\frac{p_{r}}{\rho}\right)\left(\frac{m+4 \pi p_{r} r_{\max }^{3}}{r\left(1-2 m / r_{\max }\right)}\right) .
$$

Therefore, we can still keep the radial pressure and the density continuous which will imply that,

$$
p_{r}^{\prime}\left(r_{\max }^{+}\right)=-\frac{2 \rho_{\max }}{r_{\max }} \Delta\left(r_{\max }^{-}\right) .
$$

The implications of confining the pressure anisotropy to the smallest interval possible are shown in figure 4.4 , where the anisotropy is confined to the region $r \in\left(r_{g}, r_{\max }\right]$.

\section{How does this relate to other gravastar models?}

The Mazur-Mottola model is recovered as the limiting case where $r_{g} \rightarrow r_{0} \leftarrow r_{\max }{ }^{24}$. This results in the fact that all the important anisotropy is confined in their inner thin shell. The anisotropy $\Delta \rightarrow \infty$, because $p^{\prime} \rightarrow \infty$. Effectively $\Delta$ is replaced by choosing an appropriate finite (in this case negative) surface tension $\vartheta$ and surface energy density $\sigma$ which is given by the Israel-Lanczos-Sen junction conditions $[4,51,53,68,77]$.

The second, outer thin shell which is present in the Mazur-Mottola model is not a physical necessity, but is merely a convenient way to avoid an infinitely diffuse atmosphere that would arise otherwise from the equation of state $p=\rho$. A finite

\footnotetext{
${ }^{24}$ See section (4.1.2) for more details
} 


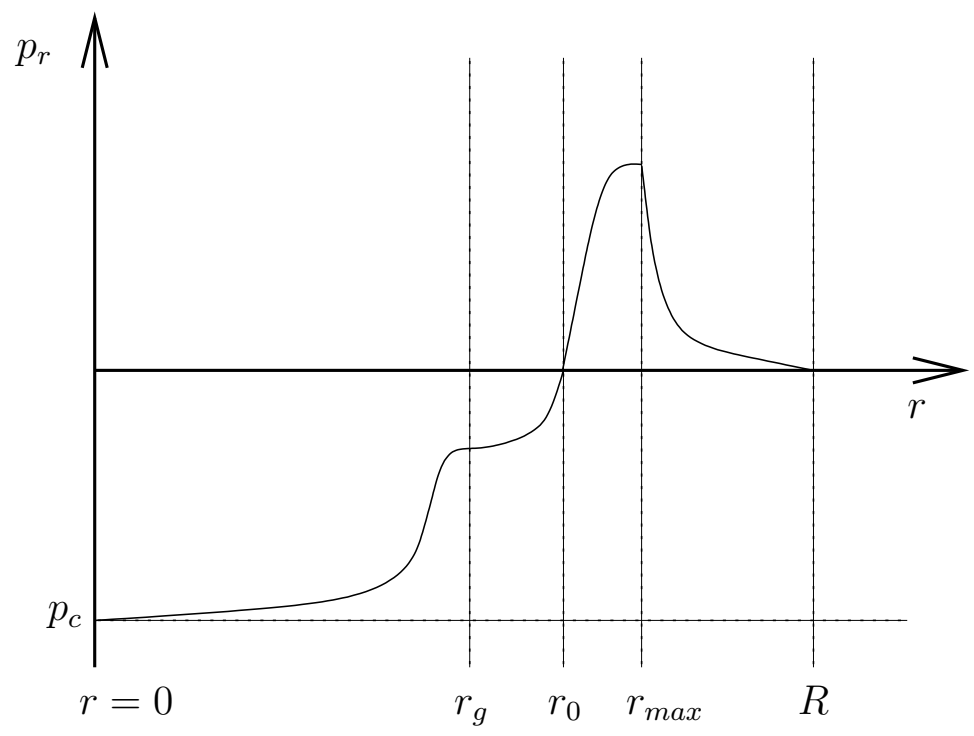

Figure 4.4: Qualitative sketch of radial pressure as a function of $r$ for a gravastar that is as near as possible to a perfect fluid. Note the inflexion point at $r_{g}$ and the kink at $r_{\max }$.

surface radius $R$ can be modelled by altering the equation of state slightly to include a finite surface density $\rho_{S}$ which is reached for vanishing pressure: $\rho(p)=\rho_{S}+p$. Then, the outer thin shell can be omitted when joining the gravastar metric onto the Schwarzschild exterior metric.

\subsubsection{Features of the anisotropic equation of state}

In the case of a perfect fluid, the geometry is completely defined by the set of differential equations (4.13), (4.19) plus initial conditions and an equation of state, which is commonly written as $\rho=\rho(p)$ or equivalently $p=p(\rho)$.

However, in this chapter we have pointed out that gravastars with perfect fluid sphere are a lost cause, and one needs to replace the isotropic TOV equation (4.19) with its anisotropic counterpart (4.21). By doing so, we also need to introduce an additional free function $\Delta(r)$. In order to close the set of equations, it is necessary to 
define two equations of state.

\section{Two equations of state}

Two equations of state must be chosen, but which criteria does one take into account?

- $p_{r}(\rho)$ and $p_{t}(\rho)$

This is a rather strong assumption that forces $p_{r}$ and $p_{t}$ to change in lock-step.

- density profile $\rho(r)$ and a single equation of state / pressure profile $p_{r}(r)$ and a single equation of state

This is the strategy adopted, for example, in [24, 25, 26, 27, 28].

- Specify any two profiles $\rho(r), p_{r}(r)$, and $p_{t}(r)$ by hand, and use the anisotropic TOV to calculate the remaining profile.

This is rather unphysical.

- finding an additional (differential) equation

This is another possibility to obtain a well defined solution, that might be motivated by some appropriate variational principle. An example would consists of minimizing the "total anisotropy"

$$
\frac{1}{R} \int_{0}^{R}(\rho \Delta)^{2} \mathrm{~d} r \Rightarrow \delta\left[\int_{0}^{R}(\rho \Delta)^{2} \mathrm{~d} r\right]=0 .
$$

No matter whether it is an equation of state for the transverse pressure or a variational principle for the total anisotropy, the extra equation should (if the gravastar model is to be even qualitatively correct) be responsible for maintaining stability of the gravastar over a wide range of total masses and central pressures. In other words, the closed set of equations must be self-regulating if it is to be physically interesting. The gravastar should shift to a new stable configuration when the total mass changes. 
The fact that, there will be regions where $\Delta>0$ in the gravastar configuration suggests that it might be most efficient to choose the two distinct "equations of state" as being equations for $\rho$ and for $\Delta$.

What variables should these equations of state depend on? It seems obvious that they should depend on the radial pressure $p_{r}$, but in view of the lower bounds on $\Delta$ in equation (4.116), it is clear that to avoid the formation of an horizon in the gravastar configuration, the equations of state should be sensitive to the compactness $2 \mathrm{~m} / \mathrm{r}$.

Therefore, we should posit equations of state of the form

$$
\rho=\rho\left(r, p_{r}, 2 m / r\right) ; \quad \Delta=\Delta\left(r, p_{r}, 2 m / r\right)
$$

and solve the paired differential equations

$$
\begin{aligned}
\frac{\mathrm{d} p_{r}(r)}{\mathrm{d} r}=- & \frac{\left[\rho\left(r, p_{r}(r), 2 m(r) / r\right)+p_{r}(r)\right]\left[2 m(r) / r+8 \pi p_{r}(r) r^{2}\right]}{2 r[1-2 m(r) / r]} \\
+ & \frac{2 \rho\left(r, p_{r}(r), 2 m(r) / r\right) \Delta\left(r, p_{r}(r), 2 m(r) / r\right)}{r} ; \\
& \frac{\mathrm{d} m(r)}{\mathrm{d} r}=4 \pi \rho\left(r, p_{r}(r), 2 m(r) / r\right) r^{2} .
\end{aligned}
$$

The success of this gravastar model depends on whether or not one can find physically realistic equations of state that take into account the effect of the compactness $2 m / r$ for large ranges of central negative pressure $p_{c}$ and total mass $M$.

\section{Potential issue:}

Some traditional relativists might argue that there is an important issue of principle, by stating that the variable $2 \mathrm{~m} / r$ is not detectable by local physics. There might also argue that an equation of state that depends on $2 \mathrm{~m} / r$ somehow violates the Einstein equivalence principle ${ }^{25}$.

But this is incorrect because $2 \mathrm{~m} / \mathrm{r}$ is certainly measurable by quasi-local effects in small but finite size regions. For any static spherically symmetric spacetime the

\footnotetext{
${ }^{25}$ See section 2 for the equivalence principle
} 
orthonormal components of the Riemann tensor are (see, for instance, [74, p 110]):

$$
\begin{aligned}
R_{\hat{t} \hat{r} \hat{t} \hat{r}} & =\frac{4 \pi}{3}\left[3\left(\rho-p_{r}+2 p_{t}\right)-2 \bar{\rho}\right] \\
R_{\hat{t} \hat{\theta} \hat{\theta} \hat{\theta}} & =\frac{4 \pi}{3}\left[3 p_{r}+\bar{\rho}\right] ; \\
R_{\hat{r} \hat{\theta} \hat{r} \hat{\theta}} & =\frac{4 \pi}{3}[3 \rho-\bar{\rho}] ; \\
R_{\hat{\theta} \hat{\phi} \hat{\theta} \hat{\phi}} & =\frac{4 \pi}{3}[2 \bar{\rho}] .
\end{aligned}
$$

The Riemann tensor is certainly measurable in finite-sized regions, so in particular $\bar{\rho}$ is measurable. Likewise $r$ is measurable in finite-sized regions, and therefore $2 \mathrm{~m} / r=$ $(8 \pi / 3) \bar{\rho} r^{2}$ is measurable.

Consequently the compactness $2 \mathrm{~m} / \mathrm{r}$ is a quasi-local measurable quantity and it can meaningfully be put into the equation of state without violating the equivalence principle.

Note that in either the Mazur-Mottola scenario $[57,58,59]$ or the Laughlin et al. scenario $[20,21]$ the gravastar material is assumed to be a quantum condensate, and therefore sensitive to non-local physics. The point is that we do not need to appeal to quantum non-locality to get $2 \mathrm{~m} / \mathrm{r}$ into the equation of state.

However, note that while this argument demonstrates that possible equations of state are at least conceivable, that is not the same as explicitly demonstrating that such equations of state actually exist.

\subsection{Results and discussion}

This chapter has hopefully served as a brief introduction to the idea of gravastars and how they are configured, as well as some of their properties.

We have discussed the qualitative features one would expect from a gravastar configuration that has finite continuous radial pressure profile everywhere and that does not have delta function transition layers (4.2). More importantly, we posit that 
- Inside the gravastar, $r<R$, the density is everywhere positive and finite.

- The central pressure is negative, $p_{c}<0$, and in fact $p_{c}=-\rho_{c}$, and the spacetime in the centre is regular.

- The spacetime is assumed to not possess an event horizon.

- We choose to enforce the WEC throughout the gravastar but impose no restrictions on other energy conditions.

- Our model describes a radial pressure that starts negative at the centre, then increases till $p_{r}=0$, becomes positive, and starts decreasing after a maximum $p_{r}=p_{\max }$ at $r=r_{\max }$ eventually reaching a second zero at the surface.

Adopting $(t, r, \theta, \phi)$ coordinates, the interior geometry for gravastars can be written in the form,

$$
d s^{2}=-\exp \left(2 \int_{r}^{\infty} g(\tilde{r}) d \tilde{r}\right) d t^{2}+\frac{d r^{2}}{(1-2 m(r) / r)}+r^{2} d \Omega^{2}
$$

where $d \Omega^{2}=d \theta^{2}+\sin ^{2} \theta d \phi^{2}$, and $m(r)$ represents the total mass-energy inside the radius $r$ integrated from the centre $r=0$ until arbitrary $r$. The gravitational acceleration $g(r)$ is written as:

$$
g(r)=\frac{4 \pi r}{3} \frac{\bar{\rho}+3 p_{r}(r)}{1-2 m(r) / r} .
$$

We have used the isotropy TOV equation where the tangential pressure is equal to the radial pressure $p_{r}=p_{t}$,

$$
\frac{d p}{d r}=-(\rho+p) g
$$

and the more general anisotropy TOV equation,

$$
\frac{d p_{r}}{d r}=-\frac{4 \pi r}{3} \frac{\left(\rho+p_{r}\right)\left(\bar{\rho}+3 p_{r}\right)}{1-2 m(r) / r}+\frac{2 \rho \Delta}{r},
$$

where we have defined the anisotropy dimensionless parameter

$$
\Delta=\frac{p_{t}-p_{r}}{\rho} .
$$


From theses specific features and the isotropy TOV equation, we have analyzed and deduced that gravastars with perfect fluid sphere do not exist, that is, gravastar-like objects must have anisotropic pressure.

We also have extracted some generic information concerning the features that the equations of state of a gravastar should have. More specifically, any gravastar-like object, that has negative central pressure $p_{c}$, must have anisotropic pressure in their "crust"; the "crust" being the region where the pressure is increasing as one moves outward and the local force of gravity is inward (4.3.1). Since the compactness is monotonic, we have, assuming the NEC,

$$
\lim _{r \rightarrow \infty} \frac{2 m(r)}{r}=\chi_{*} ; \quad \chi_{*} \in(0,1] .
$$

Not only do perfect fluid gravastars lead to inconsistencies when the isotropy TOV equation holds for finite continuous pressure, but, when the pressure is allowed to be discontinuous for the TOV equation to hold, they lead to an infinite-size infinite-mass object, or a naked singularity as the pressure exhibits a pole (4.3.2 and 4.3.3).

If we assume the DEC, we can explicitly bound the magnitude of the anisotropy, required in the crust, in terms of the local compactness $2 m(r) / r$. We have shown that,

$$
\Delta \geq \frac{1}{4} \frac{2 m / r}{1-2 m / r}>0 \quad r_{g}<r<r_{0}
$$

and

$$
0 \leq \frac{r p_{r}^{\prime}}{2 \rho}<\Delta<\frac{r p_{r}^{\prime}}{2 \rho}+\frac{1}{4} \frac{2 m / r}{1-2 m / r} \quad r_{0}<r<r_{\max } .
$$

The second inequality (4.167) is weaker than the first one (4.166), and it might still be possible to find better bounds.

The magnitude of the anisotropy becomes arbitrary large for gravastars that are sufficiently close to forming a horizon, that is,

$$
\frac{2 m}{r}>\frac{4}{5}
$$

in which case the DEC must be violated (4.4.1). 
The compactness bounds are harder to state as well: if one is prepared to ignore the DEC then it seems that the compactness can be arbitrarily close to one.

When trying to minimize the anisotropy region in the gravastar model, one has to pay the price of a discontinuity in $p_{r}^{\prime}$ and $\Delta$, and a "kink" in the radial pressure profile $p_{r}$.

We have also deduced, that for configurations that are horizon-avoiding for any total mass and negative central pressure, the equations of state of gravastar matter must depend on the local compactness $2 m(r) / r$. We posit that the equations of state are of the form

$$
\rho=\rho\left(r, p_{r}, 2 m / r\right) ; \quad \Delta=\Delta\left(r, p_{r}, 2 m / r\right) ;
$$

and that one should solve the paired differential equations

$$
\begin{aligned}
& \frac{\mathrm{d} p_{r}(r)}{\mathrm{d} r}=- \frac{\left[\rho\left(r, p_{r}(r), 2 m(r) / r\right)+p_{r}(r)\right]\left[2 m(r) / r+8 \pi p_{r}(r) r^{2}\right]}{2 r[1-2 m(r) / r]} \\
&+ \frac{2 \rho\left(r, p_{r}(r), 2 m(r) / r\right) \Delta\left(r, p_{r}(r), 2 m(r) / r\right)}{r} ; \\
& \frac{\mathrm{d} m(r)}{\mathrm{d} r}=4 \pi \rho\left(r, p_{r}(r), 2 m(r) / r\right) r^{2} .
\end{aligned}
$$

The success of this gravastar model depends on whether or not one can find physically realistic equations of state that take into account the effect of the compactness $2 \mathrm{~m} / r$. However, we have not explicitly demonstrated that such equations of state actually exists. Note that, as discussed in (4.4.3), this unusual equation of state does not violate the equivalence principle.

Even though we are personally agnostic to the existence or non-existence of gravastars, the study and understanding of their properties are important to comprehend what observational data regarding astrophysical black holes are telling us. 


\section{Chapter 5}

\section{Conclusion}

Two main problems in general relativity have been addressed in this thesis:

- The first problem is relevant to cosmology and singularities.

- The second looks at the gravastar model developed by Mazur and Mottola, a possible alternative to black holes.

Main results have already been summarized to a large extent at the end of each respective chapter. However, the main important results are highlighted below:

- Cosmology:

Three issues have been explored concerning this topic:

- We have developed a complete catalogue of the various cosmological milestones in terms of a generalized power series expansion of the FRW scale factor. This power series expansion is sufficiently general to accommodate all commonly occurring models considered in the literature.

- With the notion of a generalized power series in hand, it is possible at a purely kinematic level to address the question of when a cosmological milestone corresponds to a curvature singularity, and what type of singularity is implied. An important result is that there are only a few cases of cosmological milestones that are not polynomial curvature singularities. And the 
only two situations in which a cosmological milestone is not a derivative curvature singularity correspond to :

* an extremality event;

* a FRW geometry that smoothly asymptotes near the cosmological milestone to the Riemann-flat Milne universe.

- Finally, this definition of cosmological milestones in terms of generalized power series enables us to perform a complete model-independent check on the validity or otherwise of the classical energy conditions. In particular we provide a complete catalogue of those bangs/crunches, sudden singularities and extremality events for which the NEC, the WEC, the SEC and the DEC are satisfied. Depending on one's attitude towards the energy conditions, one could use this catalogue as a guide towards deciding on potentially interesting scenarios to investigate. In particular, the DEC is satisfied all the way to the singularity for a few range of sudden singularities $\left(\eta_{0}=0\right)$ that are sufficiently gentle.

- The gravastar model:

- We have discussed the qualitative features one would expect from a gravastar configuration that has finite continuous radial pressure profile everywhere and that does not have delta function transition layers.

- From these specific features and the isotropic TOV equation, we have analyzed and deduced that gravastars with perfect fluid sphere do not exist, that is, gravastar-like objects must have anisotropic pressure.

- We also have extracted some generic information concerning the features that the equations of state of a gravastar should have: for configurations that are horizon-avoiding for any total mass and negative central pressure, the equations of state of gravastar matter must depend on the local compactness $2 m(r) / r$. The success of this gravastar model depends on whether or not one can find physically realistic equations of state that take into account the effect of the compactness $2 m(r) / r$. 
To conclude, I would like to point out several open problems that have not yet been analyzed.

The results posited in cosmology assume an isotropic and homogeneous FRW universe. However, it would be interesting to look at what happens when assuming an inhomogeneous universe. Calculations would become much harder and not as simple as in the homogeneous case, but will we still have some energy conditions satisfied all the way through the cosmological milestones?

Concerning the gravastar model, its success depends on whether or not one can find physically realistic equations of state that take into account the effect of the compactness. However, we have not explicitly demonstrated that such equations of state actually exist. This is a challenge that we leave for the future. 


\section{Appendix A}

\section{Main spacetime metrics considered}

This appendix assembles all the main metrics that have been mentioned in this thesis.

\section{A.1 Static spacetime}

For a static spacetime, in the chosen coordinate system with arbitrary $\left\{x^{a}\right\}$, the metric components are of the form:

$$
d s^{2}=-V^{2}\left(x^{1}, x^{2}, x^{3}\right) d t^{2}+\sum_{a, b=1}^{3} g_{a b}\left(x^{1}, x^{2}, x^{3}\right) d x^{a} d x^{b}
$$

where $V^{2}=-\xi_{a} \xi^{a}$ and $\xi^{a}$ is a Killing vector field.

This metric is first mentioned in section 2.5.2.

\section{A.2 Schwarzschild exterior solution}

The Schwarzschild solution, describing static spherical symmetric vacuum spacetimes, is in standard coordinates $(t, r, \theta, \phi)$ :

$$
d s^{2}=-\left(1-\frac{2 G M}{r}\right) d t^{2}+\frac{1}{\left(1-\frac{2 G M}{r}\right)} d r^{2}+r^{2} d \Omega^{2}
$$


where $d \Omega^{2}=d \theta^{2}+\sin ^{2} \theta d \phi^{2}$. This solution is also known as the "Schwarzschild exterior solution". This is true for any spherically symmetric vacuum solution to Einstein's equations. $M$ is a parameter that can be interpreted as the conventional Newtonian mass that would be measured by studying orbits at large distances from the gravitating source.

This metric is first mentioned in section 2.5.2.

\section{A.3 Friedmann-Robertson-Walker geometry}

In cosmology, the homogeneous and isotropic cosmological model called the FriedmannRobertson-Walker geometry, (FLRW Friedmann-Lemaitre-Robertson-Walker geometry) is a good description of our universe and is derived by symmetry considerations without using the Einstein equations of general relativity. The metric is given by:

$$
d s^{2}=-d t^{2}+a(t)^{2}\left\{\frac{d r^{2}}{1-k r^{2}}+r^{2}\left[d \theta^{2}+\sin ^{2} \theta d \phi^{2}\right]\right\}
$$

where, $a(t)$ is the scale factor of the universe. There are only three values of interest for the parameter $k$ :

- $k=-1$, this corresponds to a negative curvature (for the hyperboloid)

- $k=0$, this corresponds to no curvature (flat space)

- $k=+1$, this corresponds to a positive curvature (for the 3 -sphere)

The assumptions of homogeneity and isotropy alone determine the spacetime metric up to three discrete possibilities of spatial geometry $k$ and the arbitrary positive function of the scale factor $a(t)$.

This metric is first mentioned in section 2.7. 


\section{A.4 Static spherically symmetric spacetime}

The most general static spherically symmetric line element in four dimensions can be written in the canonical form [79]:

$$
d s^{2}=-f(r) d t^{2}+h(r) d r^{2}+r^{2} d \Omega,
$$

where $d \Omega^{2}=d \theta^{2}+\sin ^{2} \theta d \phi^{2}$.

This metric is first mentioned in section 4.2.

\section{A.5 Interior of a gravastar}

Adopting $(t, r, \theta, \phi)$ coordinates we write any static spherically symmetric geometry, and in particular for the interior of a gravastar, in the form:

$$
d s^{2}=-\exp \left(2 \int_{r}^{\infty} g(\tilde{r}) d \tilde{r}\right) d t^{2}+\frac{d r^{2}}{\left(1-\frac{2 m(r)}{r}\right)}+r^{2} d \Omega^{2},
$$

where the gravitational acceleration $g(r)$ is:

$$
g(r)=\frac{4 \pi r}{3} \frac{\bar{\rho}+3 p_{r}(r)}{1-\frac{2 m(r)}{r}} .
$$

This general solution is also know as an "interior solution". Of course, the metric of the exterior of the gravastar is obviously the Schwarzschild exterior solution (A.2).

This metric is first mentioned in section 4.2.

\section{A.6 General Schwarzschild interior solution}

The Schwarzschild exterior solution in standard coordinates $(t, r, \theta, \phi)$ is given by equation (A.2). A different solution is the general Schwarzschild interior solution for values of $r$ smaller than the Schwarzschild radius $R_{\text {Schwarzschild }}$ is given by:

$$
d s^{2}=-\left(A+B \sqrt{1-\frac{2 m_{*}(r)}{r}}\right)^{2} d t^{2}+\left(\frac{1}{1-\frac{2 m_{*}(r)}{r}}\right) d r^{2}+r^{2} d \Omega^{2}
$$


where $d \Omega^{2}=d \theta^{2}+\sin ^{2} \theta d \phi^{2}$ and $m_{*}(r)=M(r / R)^{3}$. This general solution describes static spherically symmetric stars with constant density independent of $r$ from the centre to the surface of the star.

This metric is first mentioned in section 4.3.2.

\section{A.7 Schwarzschild interior solution as a function of the total mass $M$ and the surface radius $R$}

If one wishes to determine the metric of the Schwarzschild interior solution entirely as a function of the total mass $M$ and the surface radius $R$ instead of $A$ and $B$, it can be found that:

$$
\left\{\begin{array}{l}
A=\sqrt{1-\frac{2 M}{R}} \\
B=-\frac{1}{3} .
\end{array}\right.
$$

In this case the metric becomes:

$$
d s^{2}=-\left(\sqrt{1-\frac{2 M}{R}}-\frac{1}{3} \sqrt{1-\frac{2 m_{*}(r)}{r}}\right)^{2} d t^{2}+\left(\frac{1}{1-\frac{2 m_{*}(r)}{r}}\right) d r^{2}+r^{2} d \Omega^{2}
$$

where $m_{*}(r)=M(r / R)^{3}$.

This metric is first mentioned in section 4.3.2. 


\title{
Appendix B
}

\section{Necessary and sufficient conditions for big bangs, bounces, crunches, rips, sudden singularities, extremality events, and more...}

\author{
Céline Cattoën and Matt Visser \\ School of Mathematics, Statistics, and Computer Science, \\ Victoria University of Wellington, \\ P.O.Box 600, Wellington, New Zealand
}

Until recently, the physically relevant singularities occurring in FRW cosmologies had traditionally been thought to be limited to the "big bang", and possibly a "big crunch". However, over the last few years, the zoo of cosmological singularities considered in the literature has become considerably more extensive, with "big rips" and "sudden singularities" added to the mix, as well as renewed interest in non-singular cosmological events such as "bounces" and "turnarounds". In this article we present an extensive catalogue of such cosmological milestones, both at the kinematical and dynamical level. First, using generalized power series, purely kinematical definitions of these cosmological events are provided in terms of the behaviour of the scale factor $a(t)$. The notion of a "scale-factor singularity" is defined, and 
its relation to curvature singularities (polynomial and differential) is explored. Second, dynamical information is extracted by using the Friedmann equations (without assuming even the existence of any equation of state) to place constraints on whether or not the classical energy conditions are satisfied at the cosmological milestones. We use these considerations to derive necessary and sufficient conditions for the existence of cosmological milestones such as bangs, bounces, crunches, rips, sudden singularities, and extremality events. Since the classification is extremely general, and modulo certain technical assumptions complete, the corresponding results are to a high degree model-independent: In particular, we provide a characterization of the class of bangs, crunches, and sudden singularities for which the dominant energy condition is satisfied.

gr-qc/0508045; Accepted for publication in Classical and Quantum Gravity. celine.cattoen@mcs.vuw.ac.nz, matt.visser@mcs.vuw.ac.nz

Note that the full version of this paper can be found in Class.Quant.Grav.22:49134930, 2005 or there is an e-Print Archive version: gr-qc/0508045. 


\title{
Appendix C
}

\section{Gravastars must have anisotropic pressures}

\author{
Céline Cattoën, Tristan Faber, and Matt Visser \\ School of Mathematics, Statistics, and Computer Science, \\ Victoria University of Wellington, \\ P.O.Box 600, Wellington, New Zealand
}

One of the very small number of serious alternatives to the usual concept of an astrophysical black hole is the "gravastar" model developed by Mazur and Mottola; and a related phasetransition model due to Laughlin et al. We consider a generalized class of similar models that exhibit continuous pressure - without the presence of infinitesimally thin shells. By considering the usual TOV equation for static solutions with negative central pressure, we find that gravastars cannot be perfect fluids - anisotropic pressures in the "crust" of a gravastar-like object are unavoidable. The anisotropic TOV equation can then be used to bound the pressure anisotropy. The transverse stresses that support a gravastar permit a higher compactness than is given by the Buchdahl-Bondi bound for perfect fluid stars. Finally we comment on the qualitative features of the equation of state that gravastar material must have if it is to do the desired job of preventing horizon formation.

gr-qc/0505137; Published as Classical and Quantum Gravity 22 (2005) 4189-4202

celine.cattoen@mcs.vuw.ac.nz, tristan.faber@mcs.vuw.ac.nz, matt.visser@mcs.vuw.ac.nz 


\title{
Appendix D
}

\section{Effective refractive index tensor for weak-field gravity}

\author{
Petarpa Boonserm, Céline Cattoën, Tristan Faber, \\ Matt Visser, and Silke Weinfurtner \\ School of Mathematics, Statistics, and Computer Science, \\ Victoria University of Wellington, \\ P.O.Box 600, Wellington, New Zealand
}

Gravitational lensing in a weak but otherwise arbitrary gravitational field can be described in terms of a $3 \times 3$ tensor, the "effective refractive index". If the sources generating the gravitational field all have small internal fluxes, stresses, and pressures, then this tensor is automatically isotropic and the "effective refractive index" is simply a scalar that can be determined in terms of a classic result involving the Newtonian gravitational potential. In contrast if anisotropic stresses are ever important then the gravitational field acts similarly to an anisotropic crystal. We derive simple formulae for the refractive index tensor, and indicate some situations in which this will be important.

gr-qc/0411034; Published as Classical and Quantum Gravity 22 (2005) 1905-1915

petarpa.boonserm@mcs.vuw.ac.nz, celine.cattoen@mcs.vuw.ac.nz, tristan.faber@mcs.vuw.ac.nz, matt.visser@mcs.vuw.ac.nz, silke.weinfurtner@mcs.vuw.ac.nz 


\section{Bibliography}

[1] Marek A. Abramowicz, Wlodek Kluzniak, and Jean-Pierre Lasota. "No observational proof of the black-hole event-horizon". Astron. Astrophys., 396:L31-L34, 2002. [arXiv:astro-ph/0207270].

[2] L. E. Allen. "Cosmological perturbations through a simple bounce". AIP Conf. Proc., 736:182-187, 2005.

[3] Carlos Barcelo and Matt Visser. "Twilight for the energy conditions?". Int. J. Mod. Phys., D11:1553-1560, 2002. [arXiv:gr-qc/0205066].

[4] C. Barrabes and W. Israel. "Thin shells in general relativity and cosmology: The lightlike limit". Phys. Rev., D43:1129-1142, 1991.

[5] J. D. Barrow. "The premature recollapse problem in closed inflationary universes". Nucl. Phys., B296:697-709, 1988.

[6] John D. Barrow. "More general sudden singularities". Class. Quant. Grav., 21:5619-5622, 2004. [arXiv:gr-qc/0409062].

[7] John D. Barrow. "Sudden future singularities". Class. Quant. Grav., 21:L79-L82, 2004. [arXiv:gr-qc/0403084].

[8] John D. Barrow and Christos G. Tsagas. "New isotropic and anisotropic sudden singularities". Class. Quant. Grav., 22:1563-1571, 2005. [arXiv:gr-qc/0411045].

[9] J. D. Bekenstein. "Nonsingular general relativistic cosmologies". Phys. Rev., D11:2072-2075, 1975. 
[10] Neven Bilic, Gary B. Tupper, and Raoul D. Viollier. "Born-Infeld phantom gravastars". 2005. [arXiv:astro-ph/0503427].

[11] Petarpa Boonserm, Celine Cattoen, Tristan Faber, Matt Visser, and Silke Weinfurtner. "Effective refractive index tensor for weak field gravity". Class. Quant. Grav., 22:1905-1916, 2005. [arXiv:gr-qc/0411034].

[12] Mariam Bouhmadi-Lopez and Jose A. Jimenez Madrid. "Escaping the big rip?". JCAP, 0505:005, 2005. [arXiv:astro-ph/0404540].

[13] Ram Brustein, Stefano Foffa, and Avraham E. Mayo. "Causal entropy bound for non-singular cosmologies". Phys. Rev., D65:024004, 2002. [arXiv:hepth/0108098].

[14] Gregory A. Burnett. "Recollapse of the closed tolman space-times". Phys. Rev., D48:5688-5696, 1993. [arXiv:gr-qc/9308003].

[15] Hector Calderon and William A. Hiscock. "Quantum fields and "big rip" expansion singularities". Class. Quant. Grav., 22:L23-L26, 2005. [arXiv:gr-qc/0411134].

[16] R. R. Caldwell. "A phantom menace?". Phys. Lett., B545:23-29, 2002. [arXiv:astro-ph/9908168].

[17] Sean M. Carroll. "Spacetime and geometry: An introduction to general relativity". San Francisco, USA: Addison-Wesley (2004) 513 p.

[18] Celine Cattoen, Tristan Faber, and Matt Visser. "Gravastars must have anisotropic pressures". 2005. [arXiv:gr-qc/0505137].

[19] Celine Cattoen and Matt Visser. "Necessary and sufficient conditions for big bangs, bounces, crunches, rips, sudden singularities, and extremality events". 2005. [arXiv:gr-qc/0508045].

[20] G. Chapline, E. Hohlfeld, R. B. Laughlin, and D. I. Santiago. "Quantum phase transitions and the breakdown of classical general relativity". Int. J. Mod. Phys., A18:3587-3590, 2003. [arXiv:gr-qc/0012094]. 
[21] G. Chapline, R. B. Laughlin, and D. I. Santiago. "Emergent relativity and the physics of black hole interiors".

[22] Luis P. Chimento and Ruth Lazkoz. "On big rip singularities". Mod. Phys. Lett., A19:2479-2484, 2004. [arXiv:gr-qc/0405020].

[23] Ruben Curbelo, Tame Gonzalez, and Israel Quiros. "Interacting phantom energy and avoidance of the big rip singularity". 2005. [arXiv:astro-ph/0502141].

[24] I. Dymnikova. "Vacuum nonsingular black hole". Gen. Rel. Grav., 24:235-242, 1992.

[25] Irina Dymnikova. "Cosmological term as a source of mass". Class. Quant. Grav., 19:725-740, 2002. [arXiv:gr-qc/0112052].

[26] Irina Dymnikova. "Spherically symmetric space-time with the regular de Sitter center". Int. J. Mod. Phys., D12:1015-1034, 2003. [arXiv:gr-qc/0304110].

[27] Irina Dymnikova and Evgeny Galaktionov. "Stability of a vacuum nonsingular black hole". Class. Quant. Grav., 22:2331-2358, 2005. [arXiv:gr-qc/0409049].

[28] Irina G. Dymnikova. "The algebraic structure of a cosmological term in spherically symmetric solutions". Phys. Lett., B472:33-38, 2000. [arXiv:gr-qc/9912116].

[29] S. Eidelman, K.G. Hayes, K.A. Olive, M. Aguilar-Benitez, C. Amsler, D. Asner, K.S. Babu, R.M. Barnett, J. Beringer, P.R. Burchat, C.D. Carone, C. Caso, G. Conforto, O. Dahl, G. D'Ambrosio, M. Doser, J.L. Feng, T. Gherghetta, L. Gibbons, M. Goodman, C. Grab, D.E. Groom, A. Gurtu, K. Hagiwara, J.J. Hernández-Rey, K. Hikasa, K. Honscheid, H. Jawahery, C. Kolda, Kwon Y., M.L. Mangano, A.V. Manohar, J. March-Russell, A. Masoni, R. Miquel, K. Mönig, H. Murayama, K. Nakamura, S. Navas, L. Pape, C. Patrignani, A. Piepke, G. Raffelt, M. Roos, M. Tanabashi, J. Terning, N.A. Törnqvist, T.G. Trippe, P. Vogel, C.G. Wohl, R.L. Workman, W.-M. Yao, P.A. Zyla, B. Armstrong, P.S. Gee, G. Harper, K.S. Lugovsky, S.B. Lugovsky, V.S. Lugovsky, A. Rom, M. Artuso, E. Barberio, M. Battaglia, H. Bichsel, O. Biebel, P. Bloch, R.N. Cahn, 
D. Casper, A. Cattai, R.S. Chivukula, G. Cowan, T. Damour, K. Desler, M.A. Dobbs, M. Drees, A. Edwards, D.A. Edwards, V.D. Elvira, J. Erler, V.V. Ezhela, W. Fetscher, B.D. Fields, B. Foster, D. Froidevaux, M. Fukugita, T.K. Gaisser, L. Garren, H.-J. Gerber, G. Gerbier, F.J. Gilman, H.E. Haber, C. Hagmann, J. Hewett, I. Hinchliffe, C.J. Hogan, G. Höhler, P. Igo-Kemenes, J.D. Jackson, K.F. Johnson, D. Karlen, B. Kayser, D. Kirkby, S.R. Klein, K. Kleinknecht, I.G. Knowles, P. Kreitz, Yu.V. Kuyanov, O. Lahav, P. Langacker, A. Liddle, L. Littenberg, D.M. Manley, A.D. Martin, M. Narain, P. Nason, Y. Nir, J.A. Peacock, H.R. Quinn, S. Raby, B.N. Ratcliff, E.A. Razuvaev, B. Renk, G. Rolandi, M.T. Ronan, L.J. Rosenberg, C.T. Sachrajda, Y. Sakai, A.I. Sanda, S. Sarkar, M. Schmitt, O. Schneider, D. Scott, W.G. Seligman, M.H. Shaevitz, T. Sjöstrand, G.F. Smoot, S. Spanier, H. Spieler, N.J.C. Spooner, M. Srednicki, A. Stahl, T. Stanev, M. Suzuki, N.P. Tkachenko, G.H. Trilling, G. Valencia, K. van Bibber, M.G. Vincter, D. Ward, B.R. Webber, M. Whalley, L. Wolfenstein, J. Womersley, C.L. Woody, O.V. Zenin, and R.-Y. Zhu. "review of particle physics". Physics Letters B, 592:1+, 2004. url http://pdg.lbl.gov.

[30] George F. R. Ellis and Roy Maartens. "The emergent universe: Inflationary cosmology with no singularity". Class. Quant. Grav., 21:223-232, 2004. [arXiv:grqc/0211082].

[31] George F. R. Ellis, Jeff Murugan, and Christos G. Tsagas. "The emergent universe: An explicit construction". Class. Quant. Grav., 21:233-250, 2004. [arXiv:gr-qc/0307112].

[32] L. Fernandez-Jambrina and Ruth Lazkoz. "Geodesic behaviour of sudden future singularities". Phys. Rev., D70:121503, 2004. [arXiv:gr-qc/0410124].

[33] L. H. Ford. "Unstable fields and the recollapse of an open universe". Phys. Lett., A110:21, 1985.

[34] Paul H. Frampton. "Enhanced big rip without conventional dark energy". 2004. [arXiv:astro-ph/0407353]. 
[35] Paul H. Frampton and Tomo Takahashi. "Bigger rip with no dark energy". Astropart. Phys., 22:307-312, 2004. [arXiv:astro-ph/0405333].

[36] M. Gasperini, Massimo Giovannini, and G. Veneziano. "Cosmological perturbations across a curvature bounce". Nucl. Phys., B694:206-238, 2004. [arXiv:hepth/0401112].

[37] E. B. Gliner. Sov. Phys., 1966. JETP 22378.

[38] E. B. Gliner. "Inflationary universe and the vacuumlike state of physical medium". Phys. Usp., 45:213-220, 2002.

[39] Christopher Gordon and Neil Turok. "Cosmological perturbations through a general relativistic bounce". Phys. Rev., D67:123508, 2003. [arXiv:hep-th/0206138].

[40] J. Richard III Gott, M. Jurić, D. Schegel, F. Hoyle, M. Vogeley, M. Tegmark, N. Bahcall, and J. Brinkmann. "A map of the universe". Astrophys. J., 624:463, 2005. [arXiv:astro-ph/0310571].

[41] Jemal Guven and Niall O'Murchadha. "Bounds on $2 m / R$ for static spherical objects". Phys. Rev., D60:084020, 1999. [arXiv:gr-qc/9903067].

[42] B.K. Harrison, K.S. Thorne, M. Wakano, and J.A. Wheeler. "Gravitation theory and gravitational collapse". 1965. University of Chicago Press, Chicago.

[43] J. B. Hartle. "An introduction to Einstein's general relativity". 1985. San Francisco, USA: Addison-Wesley (2003) 582 p.

[44] L. Herrera, A. Di Prisco, J. Ospino, and E. Fuenmayor. "Conformally flat anisotropic spheres in general relativity". J. Math. Phys., 42:2129-2143, 2001. [arXiv:gr-qc/0102058].

[45] L. Herrera et al. "Spherically symmetric dissipative anisotropic fluids: A general study". Phys. Rev., D69:084026, 2004. [arXiv:gr-qc/0403006].

[46] L. Herrera, J. Martin, and J. Ospino. "Anisotropic geodesic fluid spheres in general relativity". J. Math. Phys., 43:4889-4897, 2002. [arXiv:gr-qc/0207040]. 
[47] David Hochberg, Carmen Molina-Paris, and Matt Visser. "Tolman wormholes violate the strong energy condition". Phys. Rev., D59:044011, 1999. [arXiv:grqc/9810029].

[48] Fiona Hoyle and Michael S. Vogeley. "Voids in the PSCz survey and the updated Zwicky catalog". Astrophys. J., 566:641, 2002. [arXiv:astro-ph/0109357].

[49] Fiona Hoyle and Michael S. Vogeley. "Voids in the 2dF galaxy redshift survey". Astrophys. J., 607:751-764, 2004. [arXiv:astro-ph/0312533].

[50] Jai-chan Hwang and Hyerim Noh. "Non-singular big-bounces and evolution of linear fluctuations". Phys. Rev., D65:124010, 2002. [arXiv:astro-ph/0112079].

[51] W. Israel. "Singular hypersurfaces and thin shells in general relativity". Nuovo Cim., B44S10:1, 1966.

[52] Kayll Lake. "Sudden future singularities in FLRW cosmologies". Class. Quant. Grav., 21:L129, 2004. [arXiv:gr-qc/0407107].

[53] K Lanczos. "Flachenhafte verteilung der materie in der einsteinschen gravitationstheorie". Ann. Phy (Leipzig), 74:518-540, 1924.

[54] S. Lefschetz. "Differential equations: Geometric theory". Dover, 1977, New York.

[55] R. A. Matzner and A. Mezzacappa. "A 3-dimensional closed universes without collapse in the 5-dimensional Kaluza-Klein theory". Phys. Rev., D32:3114-3117, 1985.

[56] R. A. Matzner and A. Mezzacappa. "Professor Wheeler and the crack of doom: Closed cosmologies in the 5-D Kaluza-Klein theory". Found. Phys., 16:227-248, 1986.

[57] Pawel O. Mazur and Emil Mottola. "Gravitational condensate stars". 2001. [arXiv:gr-qc/0109035].

[58] Pawel O. Mazur and Emil Mottola. "Dark energy and condensate stars: Casimir energy in the large". 2004. [arXiv:gr-qc/0405111]. 
[59] Pawel O. Mazur and Emil Mottola. "Gravitational vacuum condensate stars". Proc. Nat. Acad. Sci., 111:9545-9550, 2004. [arXiv:gr-qc/0407075].

[60] A. J. M. Medved. "Anatomy of a bounce". Class. Quant. Grav., 21:2749-2760, 2004. [arXiv:hep-th/0307258].

[61] John Miritzis. "The recollapse problem of closed frw models in higher-order gravity theories". 2005. [arXiv:gr-qc/0505139].

[62] Carmen Molina-Paris and Matt Visser. "Minimal conditions for the creation of a friedman- robertson-walker universe from a "bounce"”. Phys. Lett., B455:90-95, 1999. [arXiv:gr-qc/9810023].

[63] Shin'ichi Nojiri and Sergei D. Odintsov. "Quantum escape of sudden future singularity". Phys. Lett., B595:1-8, 2004. [arXiv:hep-th/0405078].

[64] Leonard Parker and Yi Wang. "Avoidance of singularities in relativity through two-body interactions". Phys. Rev., D42:1877-1883, 1990.

[65] R.D'Inverno. Introducing Einstein's Relativity. (Clarendon Press, Oxford), 2002.

[66] Varun Sahni, Hume Feldman, and Albert Stebbins. "Loitering universe". Astrophys. J., 385:1-8, 1992.

[67] Varun Sahni and Yuri Shtanov. "Did the universe loiter at high redshifts ?". Phys. Rev., D71:084018, 2005. [arXiv:astro-ph/0410221].

[68] N Sen. "Uber die grenzbedingungen des schwerefelds an unstetigen kreisflachen". Ann. Phy (Leipzig), 73:365-396, 1924.

[69] Hrvoje Stefancic. " "Expansion" around the vacuum equation of state: Sudden future singularities and asymptotic behavior". Phys. Rev., D71:084024, 2005. [arXiv:astro-ph/0411630].

[70] C. W. Misner K. S. Thorne and J. A. Wheeler. "Gravitation". (Freeman, San Fransisco), 1972. 
[71] Benjamin K. Tippett and Kayll Lake. "Energy conditions and a bounce in flrw cosmologies". 2004. [arXiv:gr-qc/0409088].

[72] G. Veneziano. "A model for the big bounce". JCAP, 0403:004, 2004. [arXiv:hepth/0312182].

[73] J. M. Virey et al. "On the determination of the deceleration parameter from supernovae data". Phys. Rev., D72:061302, 2005. [arXiv:astro-ph/0502163].

[74] Matt Visser. "Lorentzian wormholes: From Einstein to Hawking". Woodbury, USA: AIP (1995) 412 p.

[75] Matt Visser. "Cosmography: Cosmology without the Einstein equations". 2004. [arXiv:gr-qc/0411131].

[76] Matt Visser. "Jerk and the cosmological equation of state". Class. Quant. Grav., 21:2603-2616, 2004. [arXiv:gr-qc/0309109].

[77] Matt Visser and David L. Wiltshire. "Stable gravastars - an alternative to black holes?". Class. Quant. Grav., 21:1135-1152, 2004. [arXiv:gr-qc/0310107].

[78] Matt Visser and Nicolas Yunes. "Power laws, scale invariance, and generalized frobenius series: Applications to newtonian and TOV stars near criticality". Int. J. Mod. Phys., A18:3433-3468, 2003. [arXiv:gr-qc/0211001].

[79] R. M. Wald. "General relativity". Chicago, Usa: Univ. Pr. ( 1984) 491p. 


\section{Index}

affine connexion, 7

anisotropy, 93, 97, 98, 104, 120-122, 124-

$126,128,131-133$

Bianchi identity, 9, 96

big crunch, 32, 33, 36-38, 41, 42, 44, 47, $52-54,58,59,62-65,68,69,71-$ $73,87-90$

black hole, 5, 13-16, 18, 20, 91, 92, 110, 133

Christoffel symbol, 7, 12

coordinate singularity, 16

curve, 7

lightlike, 16

null, 13

timelike, 16 static universe, 40

tensor, 10, 108

emergent universe, 40

energy conditions, 17, 20, 26, 33, 35, 38, $56,57,60,62,73,89,99,131$

DEC, 17, 19, 20, 58, 68-73, 89, 102, $123,124,132,133$

NEC, 17-20, 57-64, 67, 71, 72, 89, 102, 106, 113, 116, 117, 122, 132

SEC, 17, 19, 20, 57, 65-68, 89, 102

WEC, 17-20, 57, 63, 64, 89, 98, 102, 104, 106, 131

equivalence principle, 6, 129, 130, 133

Friedmann, 11, 22, 23, 25, 28, 32, 33, 56, 57

de Sitter, 15, 92, 100, 101, 103, 107

DEC, 17, 19, 20, 58, 68-73, 89, 90, 102, $123,124,132,133$

deceleration parameter, 24, 42, 44, 46, 47, 57,88

Einstein, 5, 92

equivalence principle, 129

field equations, 10, 11, 13, 15, 18, 22, $23,26,28,56,94-96$ homogeneity, 21, 22, 24

horizon, 13, 16-18, 20, 36, 91, 93, 101, 102, 105, 112, 117, 124, 129, 131-133

Hubble

constant, 24

expansion rate, 24,25

parameter, 23, 24, 40, 42-44, 51, 88 geodesic, 6-8, 16 
isotropy, 21, 22, 24, 93, 106, 117, 120, 122, 131,132

Killing

's equation, 8

vector field, $6,8,14,15,93-95$

loitering universe, 40

manifold, 7, 8, 16

metric, 6, 8, 10, 13, 14, 18, 21, 22, 109

inverse, 7

Lorentzian, 7

Minkowski, 11

Schwarzchild, 96

Schwarzschild, 15, 16

spherical, 14

static, 14, 15

stationary, 14

symmetric, 14

tensor, 8,9

Minkowski, 15

naked singularity, 17, 93, 116, 120, 132

NEC, 17-20, 57-64, 67, 71, 72, 89, 102, 106, 113, 116, 117, 122, 132

perfect fluid, 10, 93, 116, 118, 127

sphere, 103, 104, 116, 117, 132

remaining lifetime of the universe, 80

Ricci

curvature, 11

scalar, 9, 10, 17, 47, 48 tensor, 9-13, 48, 49, 51

Riemann, 8, 54, 114

Riemannian manifold, 8

tensor, 9, 12, 16, 17, 47, 87, 88, 114$116,120,130$

scale factor, $16,22,24,27,32-34,36-39$, $41-45,48,51,52,54,55,57,60$, $63,64,66,68,70,73,87-90$

scale factor singularity, 33

Schwarzschild, 109

black hole, 16, 91

exterior solution, 13, 15, 92, 96, 98, 102,127

interior solution, 107, 109, 111-113

radius, 16, 91, 101

SEC, 17, 19, 20, 57, 65-68, 89, 102

singularity, 16

big bang, 16, 24, 27, 32, 33, 36-39, 41-44, 47, 52-54, 58, 59, 62-65, $68,69,71-73,87-90$

big crunch, 32, 33, 36-38, 41, 42, 44, 47, 52-54, 58, 59, 62-65, 68, 69, 71-73, 87-90

big rip, 17

curvature singularity, 17, 113

derivative curvature singularity, 54

extremality events, 33, 39-41, 43, 54, $56,58,59,63-65,69,72,87-89$ bounce, $32,33,39,41,54,56,68,87$ inflexion, 32, 40, 41, 54, 56, 68, 87, 125,127 
turnaround, 32, 39, 41, 54, 68, 87

naked singularity, 17, 93, 116, 120, 132

polynomial curvature singularity, 47

sudden singularity, 32, 33, 38, 39, 41,

43, 44, 47, 52, 54, 58, 59, 63-65,

$69,72,73,87-90$

universe

age of the universe, 77,79

FLRW, 22, 27, 87

FRW, 22, 27, 87

remaining lifetime of the universe, 80 ,

81

total age of the universe, $74,83-86$

WEC, 17-20, 57, 63, 64, 89, 98, 102, 104, 106,131

Weyl tensor, 9, 11, 48 